\title{
Bifurcação de Hopf \\ num Modelo de Controle Biológico
}

Danilo Braun Santos

\author{
DISSERTAÇÃO APRESENTADA \\ AO INSTITUTO DE MATEMÁTICA E ESTATÍSTICA \\ DA UNIVERSIDADE DE SÃO PAULO \\ PARA A OBTENÇÃO DO GRAU \\ DE MESTRE EM MATEMÁTICA APLICADA
}

Área de Concentração: Matemática Aplicada

Orientador: Jorge Manuel Sotomayor Tello

São Paulo, 02 de abril de 2004

O autor agradece a CAPES pelo apoio financeiro 


\title{
Bifurcação de Hopf num Modelo de Controle Biológico
}

\author{
Danilo Braun Santos
}

\author{
Este exemplar corresponde à redação \\ final da dissertação devidamente corrigida \\ e defendida por Danilo Braun Santos \\ e aprovada pela comissão julgadora.
}

Banca examinadora:

- Prof. Dr. Jorge Manuel Sotomayor Tello (orientador) (IME-USP);

- Prof. Dr. Clodoaldo Grotta Ragazzo (IME-USP);

- Prof. Dr. Luis Fernando Mello (DMC-UNIFEI). 
Aos meus pais 


\section{Agradecimentos}

Agradeço a todas as pessoas que ajudaram, direta ou indiretamente, para a realização deste trabalho. Meus sinceros agradecimentos especiais:

Ao professor Jorge Sotomayor, pelo grande incentivo e verdadeira orientação;

Ao professor Daniel Panazzolo, por suas dicas e conselhos muito eficientes;

À professora Sônia R. Garcia, pela disponibilidade e boa vontade incomparáveis e excelentíssima didática, a qual tive o prazer de constatar desde os tempos de graduação;

Aos meus pais, Abelardo Pinto Santos e Maria Cecília Braun, por tudo o que são e todo apoio nessa e noutras jornadas;

À minha mulher, Cecília Galvani, pelo carinho, compreensão e todas as revisões ortográficas; Aos amigos, Jocirei, Cláudio e Márcio, que contrubuiram com ajudas significativas nos meus estudos;

Aos amigos Renato e Alexandre, por suas ajudas em Latex. 


\section{Resumo}

Estudamos um sistema de equações diferenciais ordinárias composto por quatro equações não lineares acopladas. Tal sistema representa um modelo de interação biológica entre duas espécies de insetos. Analisamos o surgimento de uma bifurcação de Hopf neste sistema. Para tanto, apresentamos um método para estudar essa bifurcação em sistemas $n$-dimensionais, capaz de verificar as condições de Hopf, de não degenerescência e de transversalidade, que garantem o surgimento de uma órbita periódica. Apresentamos algumas simulações numéricas para corroborar com a análise aqui desenvolvida. 


\section{Abstract}

We study a system of ordinary differential equations composed of four coupled nonlinear ones. Such system represents a model for biological interaction between two species of insects. We analyse the appearence of a Hopf bifurcation in this system. To this end, we present a method for studying this bifurcation in $n$-dimentional systems, suitable to verify the Hopf conditions, of nondegeneracy and of transversality, which guarantee the appearence of a periodic orbit. We present some numerical simulations for corroborating the analysis developed here. 


\section{Sumário}

$\begin{array}{ll}\text { Lista de Figuras } & 3\end{array}$

$\begin{array}{ll}\text { Lista de Tabelas } & 5\end{array}$

$\begin{array}{ll}\text { Introdução } & 7\end{array}$

1 Considerações Biológicas 11

1.1 As espécies . . . . . . . . . . . . . . . . . . . . 11

1.1 .1 A praga: Phyllocnistis citrella ............... 11

1.1 .2 O parasito: Galeopsomya fausta . . . . . . . . . . . . 12

$1.2 \mathrm{O}$ modelo . . . . . . . . . . . . . . . . . . 13

1.2 .1 Descrição do modelo . . . . . . . . . . . . . . 13

1.2.2 Um modelo de crescimento . . . . . . . . . . . . . . . 14

1.2 .3 A interação entre as espécies . . . . . . . . . . . . . . 15

2 Bifurcação de Hopf $\quad 19$

2.1 Bifurcação de Hopf genérica . . . . . . . . . . . . . . . . . . 23

2.2 Método da projeção . . . . . . . . . . . . . . . . . . . . 38

3 Estudo Qualitativo do Modelo 49

3.1 Estudo das equações desacopladas . . . . . . . . . . . . . . . 50

3.2 Os pontos de equilíbrio . . . . . . . . . . . . . . . . 57

3.3 Análise do espectro . . . . . . . . . . . . . . 63

$3.3 .1 \quad\left(\mathbf{P}_{1}, \mathbf{M}_{1}, \mathrm{~L}_{1}, \mathrm{G}_{1}\right) \ldots \ldots \ldots \ldots$ 
$3.3 .2\left(\mathrm{P}_{2}, \mathrm{M}_{2}, \mathrm{~L}_{2}, \mathrm{G}_{2}\right) \ldots \ldots \ldots 6$

$3.3 .3 \quad\left(\mathrm{P}_{3}, \mathrm{M}_{3}, \mathrm{~L}_{3}, \mathrm{G}_{3}\right) \ldots \ldots \ldots \ldots$

$3.3 .4\left(\mathbf{P}_{4}, \mathbf{M}_{4}, \mathbf{L}_{4}, \mathbf{G}_{4}\right) \ldots \ldots \ldots \ldots$

3.4 Condição de Hopf . . . . . . . . . . . . . . . . . . 77

3.5 Resumo . . . . . . . . . . . . . . . . . . 79

4 Simulações Numéricas $\quad 81$

4.1 Estudo de um caso específico . . . . . . . . . . . . . . . . . 82

4.1 .1 As equações desacopladas . . . . . . . . . . . . . . . 82

4.1 .2 Cálculo do valor de bifurcação . . . . . . . . . . . . . 86

4.1 .3 Os pontos de equilíbrio e os autovalores . . . . . . . . . . 87

4.1.4 Cálculo do coeficiente de Lyapunov . . . . . . . . . . . . . . . . . 91

4.1 .5 A condição de transversalidade . . . . . . . . . . . . . 100

4.1.6 Simulação numérica de diversas condições de campo . . . . . . . . . 102

4.1 .7 Escolha do vetor $q \ldots \ldots \ldots \ldots \ldots . \ldots \ldots$

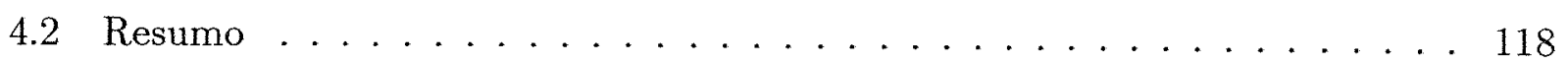

$\begin{array}{ll}\text { Conclusão } & 119\end{array}$

$\begin{array}{ll}\text { Referências Bibliográficas } & 121\end{array}$ 


\section{Lista de Figuras}

2.1 Retrato de fase da bifurcação de Hopf . . . . . . . . . . . . . . . . . . 21

2.2 Mapeamento de Poincaré para a bifurcação de Hopf . . . . . . . . . . . . . 24

2.3 Ponto fixo do mapeamento de retorno . . . . . . . . . . . . . 25

2.4 Construção do homeomorfismo próximo à bifurcação de Hopf . . . . . . . . . 27

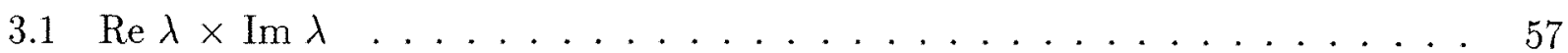

3.2 Retrato $P M \times L G \ldots \ldots \ldots \ldots \ldots \ldots$

4.1 Retrato de fase $P \times M \ldots \ldots \ldots \ldots$

4.2 Retrato de fase $L \times G \ldots \ldots \ldots \ldots \ldots \ldots \ldots$

$4.3 M \times G \mid$ próximo ao equilíbrio $\mid n=300 \ldots \ldots \ldots \ldots$

$4.4 P \times L \mid$ próximo ao equilíbrio $\mid n=300 \ldots \ldots \ldots \ldots \ldots$

$4.5 M \times G \mid$ distante do equilíbrio $\mid n=23 \ldots \ldots \ldots$

$4.6 P \times L \mid$ distante do equilíbrio $\mid n=23 \ldots \ldots \ldots \ldots$

$4.7 M \times G\left|k<k_{0}\right|$ situação (i) $\left|G_{0}=10\right| n=34 \ldots \ldots \ldots$. . . . . . . 107

$4.8 M \times G\left|k<k_{0}\right| \operatorname{situação~(i)~}\left|G_{0}=100\right| n=50 \ldots \ldots$. . . . . . . 107

$4.9 M \times G\left|k<k_{0}\right|$ situação (i) $\left|G_{0}=300\right| n=3 \ldots \ldots \ldots$

$4.10 M \times G\left|k<k_{0}\right| \operatorname{situação~(ii)~}\left|G_{0}=10\right| n=18 \ldots \ldots \ldots$. . . . . . 108

$4.11 M \times G\left|k<k_{0}\right| \operatorname{situação~(iii)~}\left|G_{0}=10\right| n=11 \ldots \ldots . \ldots . \ldots 109$

$4.12 M \times G\left|k>k_{0}\right|$ situação (i) $\left|G_{0}=10\right| n=300 \ldots \ldots$. . . . . . . 109

$4.13 M \times G\left|k>k_{0}\right| \operatorname{situação~(ii)~}\left|G_{0}=10\right| n=21 \ldots \ldots \ldots$

$4.14 M \times G\left|k>k_{0}\right| \operatorname{situação~(ii)~}\left|G_{0}=30\right| n=300 \ldots \ldots \ldots . \ldots 110$

$4.15 M \times G\left|k>k_{0}\right|$ situação (iii) $\left|G_{0}=30\right| n=7 \ldots \ldots \ldots 111$ 
$4.16 M \times G\left|k>k_{0}\right| \operatorname{situação~(iii)~}\left|G_{0}=50\right| n=14 \ldots \ldots \ldots \ldots \ldots$

$4.17 M \times G\left|k>k_{0}\right|$ situação (iii) $\left|G_{0}=70\right| n=300 \ldots \ldots \ldots \ldots 12$

$4.18 M \times G\left|k>k_{0}\right| \operatorname{situação~(iii)~}\left|G_{0}=600\right| n=2 \ldots \ldots \ldots \ldots \ldots$ 


\section{Lista de Tabelas}

1.1 Significado dos parâmetros biológicos . . . . . . . . . . . . 16

4.1 Sequência de comandos do Maple para o sistema (S) desacoplado . . . . . . 85

4.2 Sequência de comandos do Maple para cálculo do valor de bifurcação . . . . 87

4.3 Sequência de comandos do Maple para cálculo dos autovalores nos pontos $\left(P_{1}, M_{1}, L_{1}, G_{1}\right),\left(P_{2}, M_{2}, L_{2}, G_{2}\right)$ e $\left(P_{3}, M_{3}, L_{3}, G_{3}\right) \ldots \ldots \ldots$

4.4 Sequência de comandos do Maple para cálculo dos autovetores $p$ e $q$. . . . 98

4.5 Primeira sequência de comandos do Maple para cálculo do coeficiente de Lya-

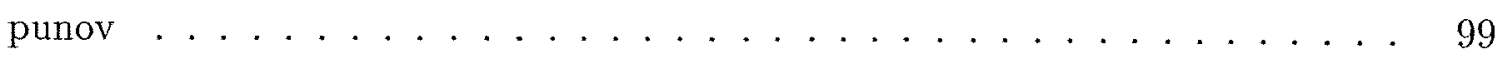

4.6 Segunda sequência de comandos do Maple para cálculo do coeficiente de Lyapunov ................................. 100

4.7 Sequência de comandos do Maple para o cálculo de $\gamma^{\prime}\left(k_{0}\right) \ldots \ldots$. . . . . 101

4.8 Sequência de comandos do Maple para a plotagem dos gráficos . . . . . . . 113

4.9 O coeficiente de Lyapunov para vários tamanhos de $q \ldots \ldots$. . . . . . 115

4.10 Sequência de comandos com tamanho do vetor $q$ aumentado . . . . . . . . 116

4.11 Sequência de comandos com tamanho do vetor $q$ diminuido . . . . . . . . 117 


\section{Introdução}

Um dos grandes desafios da humanidade tem sido explicar cientificamente os fenômenos da natureza. Aqueles que se apresentam ao estudo matemático são, em sua maioria, excessivamente complexos e impossíveis de serem analizados em todos os detalhes. O método científico analítico, iniciado com Galileu (1564 - 1642), consiste em restringir e isolar o campo de estudo de forma apropriada de modo que o problema seja tratável e, ao mesmo tempo, manter sua relevância. Seguindo essa idéia desenvolveremos o presente trabalho.

Formulado por Lotka e Volterra, por volta de 1925, um dos primeiros exemplos de interação biológica entre presa e predador, foi descrito pelo sistema

$$
\left\{\begin{array}{l}
\frac{d x}{d t}=a x-\alpha x y \\
\frac{d y}{d t}=-b y+\beta x y
\end{array}\right.
$$

onde $x(t)$ e $y(t)$ denotam, respectivamente, as densidades populacionais da presa e do predador em função do tempo. Os parâmetros $a, \alpha, b$ e $\beta$ são constantes positivas, sendo $a$ a taxa de crescimento das presas, $b$ a taxa de mortalidade dos predadores e $\alpha, \beta$ o nível de interação entre as duas espécies, ver Sotomayor [17]. Com o passar dos anos muitas adaptações e avanços foram obtidos, de forma que cada vez mais específicos e complexos se tornaram os modelos biomatemáticos.

O sistema que estudaremos é composto por quatro equações diferenciais não lineares e acopladas

$$
\left\{\begin{array}{l}
\frac{d P}{d t}=\phi\left(1-\frac{M}{c}\right) M-(\alpha+\beta) P-k P G \\
\frac{d M}{d t}=\alpha P-\mu M \\
\frac{d L}{d t}=\phi^{\prime}\left(1-\frac{G}{c^{\prime}}\right) G-\left(\alpha^{\prime}+\beta^{\prime}\right) L+k^{\prime} P G \\
\frac{d G}{d t}=\alpha^{\prime} L-\mu^{\prime} G
\end{array}\right.
$$


onde $P, M, L$ e $G$ representam populações de insetos.

Esse sistema visa modelar o comportamento de duas espécies competidoras em interação. Uma dessas espécies é uma praga, conhecida como larva minadora da folha de citros, responsável por infestações em laranjais com grande potencial de danos à citricultura nacional devido à sua associação com o cancro cítrico. O manejo dessa praga, de modo a manter o nível populacional baixo e evitar prejuízos e danos econômicos, motiva a compreensão do problema. O sistema presa-predador descrito foi encontrado em uma publicação de autoria de Hyun e Ternes [11].

O controle biológico de pragas visa regular populações de espécies e vem sendo utilizado pelo homem a seu favor. A inserção de um parasita inimigo de uma espécie pode controlar o nível de infestação de uma praga, sucumbí-la ou nem mesmo afetá-la. Conhecer em que condições atingem-se os objetivos desejados é o principal motivo de tais modelos.

Em geral, nos modelos presa-predador, propostos na literatura pertinente, procura-se provar a existência de um ponto de equilíbrio estável ou um ciclo limite estável de modo a fornecer uma explicação satisfatória para uma coexistência duradoura entre as comunidades biológicas.

Seguindo o propósito de se compreender melhor os modelos biomatemáticos, neste trabalho estudaremos o processo de acoplamento e o comportamento das soluções do sistema de equações diferenciais descrito, mostrando a ocorrência de uma bifurcação de Hopf nesse modelo presa-predador. Como consequência teremos o surgimento de um ciclo limite no gráfico que representa a dinâmica das populações das espécies em interação.

Primeiramente, no capítulo 1, daremos uma descrição biológica das espécies envolvidas e o processo de obtenção das equações que compõem o modelo, até chegarmos ao sistema descrito anteriormente, onde os parâmetros $k$ e $k^{\prime}$ desempenham papel importante pois descrevem o nível de interação entre as espécies.

No capítulo 2 definiremos uma bifurcação de Hopf bidimensional e apresentaremos um método para se calcular esta bifurcação em sistemas com dimensão finita qualquer. Isso será necessário uma vez que o modelo proposto está inserido em um espaço de dimensão 4.

Estudaremos, no capítulo 3, qualitativamente este modelo. O comportamento das equações desacopladas $\left(k=k^{\prime}=0\right)$, os seus pontos de equilíbrio, os autovalores da matriz Ja- 
cobiana em cada um desses pontos e, finalmente, um estudo acerca do surgimento de uma bifurcação de Hopf serão temas abordados.

No último capítulo faremos, então, algumas simulações numéricas para verificar os resultados e confirmar o surgimento da bifurcação de Hopf para um conjunto específico de dados. 


\section{Capítulo 1}

\section{Considerações Biológicas}

Neste capítulo colocaremos o problema biológico que o presente trabalho pretende estudar. Procuraremos abordar todas as hipóteses relevantes, primeiramente descrevendo o comportamento das espécies envolvidas na relação de parasitismo e, em seguida, o processo de construção das equações que modelam a interação entre essas espécies. O conteúdo biológico e os dados aqui tratados foram obtidos em Hyun e Ternes [11] e Ternes [19].

\subsection{As espécies}

Nesta seção serão apresentadas as espécies relevantes ao estudo do problema: Phyllocnistis citrella (a praga) e Galeopsomya fausta (o parasito).

\subsubsection{A praga: Phyllocnistis citrella}

A Phyllocnistis citrella (Lepidoptera: Gracillariidae) é um inseto de origem asiática conhecido popularmente por larva minadora da folha de citros ou apenas larva minadora. O inseto adulto é uma micromariposa que efetua a postura preferencialmente na superfície inferior de folhas novas de brotações de plantas cítricas. Após a eclosão, as larvas penetram no tecido foliar, onde permanecerão durante todo o seu desenvolvimento larval, passando a se alimentar da seiva e com isso construindo minas ou galerias no formato de serpentina, normalmente localizadas na superfície superior das folhas, salvo em altas infestações, onde ambos os lados 
passam a apresentar os sintomas do ataque. A pupação ocorrerá dentro de tais minas, em uma célula pupal localizada na margem da folha.

Os danos provocados pelas larvas às folhas, além de prejudicar a fotossíntese, favorecem a infecção da planta por agentes fitopatogênicos. Doenças como o cancro cítrico, causada pela bactéria Xanthomonas axonopodis pv. citri, podem, então, desenvolver-se. O cancro cítrico provoca lesões nos ramos, folhas e frutos, causando quedas na produção ou mesmo sua perda total, uma vez que, por lei, pomares contaminados são proibídos de comercializar suas produções, Fundecitrus [7]. Segundo Chagas [4], levantamentos realizados em condições de campo demonstram um índice de infestação do cancro em folhas de citros por volta de $75 \%$, quando a larva minadora esta presente.

A primeira ocorrência da larva minadora no Brasil data de 1996, tendo sido registrada em viveiros da região de Campinas [8]. Dados publicados pela Fundecitrus apontam 45 focos de cancro espalhados em 22 municípios produtores de citros, nos Estados de São Paulo e sul de Minas Gerais, neste ano de 1996. Em 1999 estes números apresentam grave aumento, sendo 4.180 focos de cancro em 132 municípios, totalizando quase 300 mil plantas contaminadas. Uma intensa campanha para a erradicação do cancro cítrico vem sendo adotada pelas autoridades competentes, de modo que em 2002 e 2003 um total de aproximadamente 450 mil plantas contaminadas foram eliminadas. Estima-se que nos últimos dois anos o cancro cítrico causou prejuízos da ordem de 300 milhões de reais, Fundecitros [8].

\subsubsection{O parasito: Galeopsomya fausta}

Espécie nativa de vários países da América do Sul e Central, a Galeopsomya fausta (Hymenoptera: Eulophidae) é o principal parasito nativo da $P$. citrella, chegando a $90 \%$ dos parasitos nativos recolhidos em campo no Estado de São Paulo. A G. fausta é um ectoparasito (se desenvolve fora do hospedeiro) das fases de pupa $(87,7 \%)$, pré-pupa $(9,8 \%)$ e larvas $(2,3 \%)$, Cobo Nuñez [5].

A fêmea da $G$. fausta ovipõe sobre a câmara pupal da $P$. citrella e, durante o seu desenvolvimento, a larva da $G$. fausta se alimenta da pupa da $P$. citrella, causando a sua morte. A espécie é caracterizada por um canibalismo intra-específico, quando vários ovos são depositados ao mesmo tempo, a larva originada do primeiro ovo a eclodir alimenta-se 
dos restantes, ver LaSalle e Peña [13].

Estudos sobre a fecundidade mostraram que a quantidade de ovos por dia varia com a temperatura, sendo 5,5 ovos por fêmea a $30^{\circ} \mathrm{C}, 10,25$ ovos por fêmea a $25^{\circ} \mathrm{C}$ e quase nula a $15^{\circ} \mathrm{C}$. Essa espécie apresenta uma reprodução do tipo partenogênese telítoca (óvulo não fecundado produz apenas fêmeas). Das análises de LaSalle e Peña [13], de 74 espécies recebidas da Colômbia, apenas 6 eram machos e, das 150 espécies provenientes do Brasil, todas eram fêmeas. A população dessa espécie mantém um crescimento natural independente do nível populacional da praga.

\subsection{O modelo}

\subsubsection{Descrição do modelo}

O modelo matemático (sugerido por Hyun e Ternes [11] e Ternes [19]) visa descrever a dinâmica populacional quando da interação entre a $P$. citrella, o inseto-praga e a $G$. fausta, o parasito. Para tanto utilizou-se o modelo compartimental, onde as principais fases do ciclo de vida neste processo de parasitismo, as fases de larva, pupa e inseto adulto, são abordadas no sistema. Por exemplo, ao se relevar para uma dada espécie apenas os estágios de larva e adulto, a fase de pupa será englobada dentro de parâmetros apropriados. Dessa forma ao se considerar a taxa de passagem da fase de larva para a fase de adulto é incluso nessa taxa a fase de pupa.

O modelo representará a população de fêmeas ao longo do tempo. Se necessário, a população de machos pode ser calculada de acordo com a razão sexual da espécie. A fase de larva não é subdividida, considera-se uma fase larval única. Do mesmo modo as fases de pré-pupa e pupa são identificadas em um mesmo estágio.

Nas taxas de natalidade foram englobados conjuntamente os períodos de cópula, préoviposição, maturação e viabilidade dos ovos.

Considera-se também que a umidade relativa não afeta os parâmetros do modelo, tanto para a praga quanto para o parasito.

Outras hipóteses que foram relevantes na construção do modelo podem ser vistas de modo mais detalhado em [19]. 


\subsubsection{Um modelo de crescimento}

Visando descrever o fenômeno biológico do crescimento de uma população de insetos, esse modelo considera a espécie subdividida em duas etapas do seu ciclo de vida, uma fase intermediária $(\mathrm{x})$ e outra adulta $(\mathrm{y})$.

Tal modelo será então descrito pelo sistema de equações diferenciais ordinárias:

$$
\left\{\begin{array}{l}
\frac{d x}{d t}=\Phi(y) y-(\alpha+\beta) x \\
\frac{d y}{d t}=\alpha x-\mu y
\end{array}\right.
$$

onde a função $\Phi$ representa a taxa per capita de crescimento da população, $\alpha, \beta$ e $\mu$ são, respectivamente, taxa de passagem da fase intermediária para a fase adulta, mortalidade do inseto na sua fase intermediária e mortalidade do inseto adulto.

Os parâmetros $\alpha, \beta$ e $\mu$ podem ser medidos em experimentos de campo ou mesmo encontrados na literatura. Já para obter uma expressão para a função $\Phi$, algumas hipóteses foram levantadas, uma vez que não existem estudos específicos sobre as espécies consideradas. Das três diferentes expressões analíticas para essa função que foram discutidas em Ternes [19], escolhemos uma dessas expressões para um estudo detalhado do modelo.

A variante que nos interessa aqui representa o crescimento natural da população tendo como fator limitante a capacidade do meio-ambiente, isto é, o crescimento populacional é controlado pela saturação do meio. Dessa forma a função fica caracterizada por

$$
\Phi(y)=\phi(1-y / c)
$$

onde $\phi$ é a taxa de criação de indivíduos da fase $\mathrm{x}$ (intermediária) e o parâmetro $c(c>0)$ representa o limite superior que a população y pode atingir devido a capacidade do meio.

Para darmos um sentido biológico a esta taxa de criação de indivíduos $(\phi)$, temos de considerar antes qual a fase intermediária tratada. Por exemplo, se a fase intermediária for a fase larval, a taxa de criação pode ser interpretada como sendo a taxa de oviposição das fêmeas fertilizadas.

Assim, o sistema fica dado por 


$$
\left\{\begin{array}{l}
\frac{d x}{d t}=\phi(1-y / c) y-(\alpha+\beta) x \\
\frac{d y}{d t}=\alpha x-\mu y
\end{array}\right.
$$

\subsubsection{A interação entre as espécies}

Construiremos agora o modelo da variação populacional das duas espécies em interação, baseando-se no modelo de crescimento de uma população isolada visto na subseção anterior.

Para a espécie Phyllocnistis citrella, a praga, designaremos por $\mathbf{M}$ a população da fase adulta e por $\mathbf{P}$ a fase de pupa. Para o parasito, Galeopsomya fausta, chamaremos de $\mathbf{G}$ a população na fase adulta e de $\mathbf{L}$ a fase larval. Como dissemos antes, ao considerarmos apenas dois estágios para cada espécie significa que as outras fases do seu ciclo de vida estão sendo englobadas nos parâmetros do sistema, no caso as fases de larva para o inseto-praga e de pupa para o parasito. Note que esta simplificação pode ser feita pois a interação entre as espécies ocorre, principalmente, quando o parasito na fase adulta (G) ovipõe sobre a pupa do inseto-praga $(\mathrm{P})$, ver seção 1.1.2.

Portanto temos que o sistema resultante será dado por

$$
\left\{\begin{array}{l}
\frac{d P}{d t}=\phi\left(1-\frac{M}{c}\right) M-(\alpha+\beta) P-f(P, G) \\
\frac{d M}{d t}=\alpha P-\mu M \\
\frac{d L}{d t}=\phi^{\prime}\left(1-\frac{G}{c^{\prime}}\right) G-\left(\alpha^{\prime}+\beta^{\prime}\right) L+g(P, G) \\
\frac{d G}{d t}=\alpha^{\prime} L-\mu^{\prime} G
\end{array}\right.
$$

onde as funções $f(P, G)$ e $g(P, G)$ representam a relação de parasitismo entre as duas espécies.

Os parâmetros $\alpha, \beta, \mu, \phi$ e $c$ referem-se a praga $P$. citrella e os parâmetros $\alpha^{\prime}, \beta^{\prime}, \mu^{\prime}, \phi^{\prime}$ e $c^{\prime}$ referem-se ao parasito G. fausta. Seus significados são apresentados na tabela (1.1).

Ressaltamos que os parâmetros $\alpha, \beta, \mu, \phi, \alpha^{\prime}, \beta^{\prime}, \mu^{\prime}, \phi^{\prime}$, possuem todos dimensão tempo ${ }^{-1}$ e que, por comodidade, apresentaremos os parâmetros e seus respectivos valores sem a informação da unidade. Os parâmetros $c$ e $c^{\prime}$ possuem dimensão insetos por talhão. "Compreendese por talhão a quantidade de plantas delimitadas de outras existentes no mesmo pomar separado por arruamentos, por estradas, por carreadores ou outro meio qualquer empregado pelo proprietário para identificá-lo ou distinguí-lo de outros agrupamentos, com largura superior ao espaçamento entre linhas" [16]. 


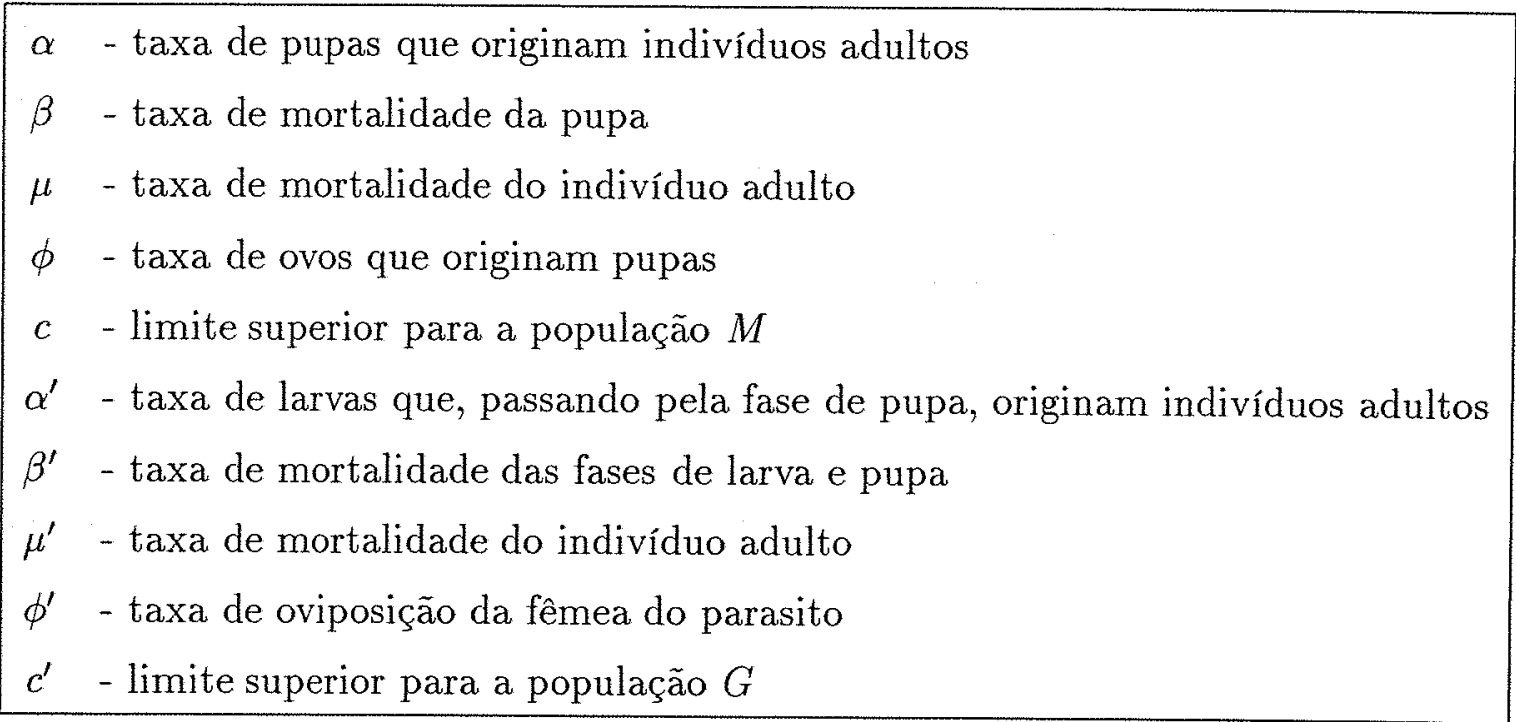

Tabela 1.1: Significado dos parâmetros biológicos

Assumindo que exista um encontro aleatório entre as duas espécies, mais especificamente entre o adulto do parasito e a pupa da praga, e que desse encontro ocorre um decréscimo da população de pupas da praga, proporcional a uma taxa $k$ e que a população do parasito se beneficia, proporcionalmente a uma taxa $k^{\prime}$, podemos aproximar as funções $f$ e $g$, que descrevem essa interação, por

$$
\left\{\begin{array}{l}
f(P, G)=k P G \\
g(P, G)=k^{\prime} P G
\end{array} .\right.
$$

Essas aproximações são razoavelmente boas quando as populações $P$ e $G$ são abundantes, o que nem sempre é verdade.

Desse modo o sistema fica finalmente dado por

$$
\left\{\begin{array}{l}
\frac{d P}{d t}=\phi\left(1-\frac{M}{c}\right) M-(\alpha+\beta) P-k P G \\
\frac{d M}{d t}=\alpha P-\mu M \\
\frac{d L}{d t}=\phi^{\prime}\left(1-\frac{G}{c^{\prime}}\right) G-\left(\alpha^{\prime}+\beta^{\prime}\right) L+k^{\prime} P G \\
\frac{d G}{d t}=\alpha^{\prime} L-\mu^{\prime} G
\end{array},\right.
$$

o qual denotaremos, a partir de agora, de sistema (S).

Observação 1.2.1 Todos os parâmetros,

$$
\alpha, \beta, \mu, \phi, c, k, \alpha^{\prime}, \beta^{\prime}, \mu^{\prime}, \phi^{\prime}, c^{\prime}, k^{\prime}
$$


são positivos.

Observação 1.2.2 Será razoável supormos que $k \geq k^{\prime}$, uma vez que no processo de parasitismo a larva da G. fausta leva à morte da pupa da P. citrella e, portanto, o fator de prejuizo à população da praga deverá ser maior do que o fator de benefício para a população do parasito. 


\section{Capítulo 2}

\section{Bifurcação de Hopf}

Este capítulo tem por objetivo estudar a bifurcação de Hopf. Inicialmente trataremos dos sistemas bidimensionais onde o conceito de bifurcação de Hopf é bastante conhecido para posteriormente estudá-lo em um contexto mais amplo, para sistemas $n$-dimensionais. As definições e o método de projeção que apresentaremos no corrente capítulo foram baseados no livro de Kuznetsov [12].

Utilizaremos a terminologia suave para nos referirmos às funções onde a classe de diferenciabilidade é suficientemente grande.

$$
f \text { suave } \Leftrightarrow f \in \mathcal{C}^{n},
$$

com $n$ suficientemente grande. Quando acharmos necessário explicitar a classe de diferenciabilidade faremos menção a respeito.

A notação $f(x)=O\left(\|x\|^{n}\right)$ representará uma função suave cuja expansão de Taylor em $x$ inicia-se com os termos de ordem $n$ (ou superiores).

Seguem algumas definições que usaremos no decorrer deste capítulo:

Definição 2.0.1 Um ponto singular $x_{0}$ do sistema

$$
\dot{x}=f(x),
$$

com $f$ suave e $x \in \mathbb{R}^{n}$, é chamado de hiperbólico se todos os autovalores de $J\left(x_{0}\right)$ têm parte real diferente de zero, onde $J\left(x_{0}\right)=D f\left(x_{0}\right)$ representa a matriz Jacobiana de $f(x)$ no ponto $x_{0}$. 
Definição 2.0.2 Um ponto singular hiperbólico $x_{0}$ do sistema

$$
\dot{x}=f(x),
$$

com $f$ suave e $x \in \mathbb{R}^{2}$, chama-se foco se $J\left(x_{0}\right)$ possuir dois autovalores complexos. Se a parte real destes autovalores for negativa chamaremos $x_{0}$ de um foco atrator. Se a parte real dos autovalores for positiva de um foco repulsor.

Considere o seguinte sistema de equações diferenciais dependendo do parâmetro $\xi$ :

$$
\left(\begin{array}{c}
\dot{x_{1}} \\
\dot{x_{2}}
\end{array}\right)=\left(\begin{array}{cc}
\xi & -1 \\
1 & \xi
\end{array}\right)\left(\begin{array}{l}
x_{1} \\
x_{2}
\end{array}\right) \pm\left(x_{1}^{2}+x_{2}^{2}\right)\left(\begin{array}{c}
x_{1} \\
x_{2}
\end{array}\right) \text {. }
$$

Para qualquer que seja $\xi \in \mathbb{R}$, o ponto $\left(x_{1}, x_{2}\right)=(0,0)$ é equilíbrio desse sistema com a matriz Jacobiana dada por

$$
A=\left(\begin{array}{cc}
\xi & -1 \\
1 & \xi
\end{array}\right)
$$

que possui autovalores $\lambda_{1}=\xi+i$ e $\lambda_{2}=\xi-i$. Introduzindo a variável complexa $z=x_{1}+i x_{2}$, como

$$
\dot{x_{1}}=\xi x_{1}-x_{2} \pm x_{1}\left(x_{1}^{2}+x_{2}^{2}\right)
$$

$\mathrm{e}$

$$
\dot{x_{2}}=x_{1}+\xi x_{2} \pm x_{2}\left(x_{1}^{2}+x_{2}^{2}\right)
$$

temos:

$$
\dot{z}=\dot{x_{1}}+i \dot{x_{2}}=\xi\left(x_{1}+i x_{2}\right)+i\left(x_{1}+i x_{2}\right) \pm\left(x_{1}+i x_{2}\right)\left(x_{1}{ }^{2}+x_{2}{ }^{2}\right) .
$$

Podemos então reescrever (2.1), na sua forma complexa, por:

$$
\dot{z}=(\xi+i) z \pm z|z|^{2}
$$

Usando agora a representação $z=\rho e^{i \theta}$, obtemos:

$$
\dot{z}=\dot{\rho} e^{i \theta}+\rho i \dot{\theta} e^{i \theta}
$$


e, portanto

$$
\dot{\rho} e^{i \theta}+\rho i \dot{\theta} e^{i \theta}=\rho e^{i \theta}\left(\xi+i \pm \rho^{2}\right) .
$$

Podemos, então, escrever a equação (2.2) em sua forma polar:

$$
\left\{\begin{array}{l}
\dot{\rho}=\rho\left(\xi \pm \rho^{2}\right) \\
\dot{\theta}=1
\end{array} .\right.
$$

Da primeira equação em (2.3), podemos perceber que $\rho=0$ é um ponto de equilíbrio para qualquer valor de $\xi$ (obviamente só consideraremos $\rho \geq 0$ ). Outro ponto de equilíbrio surgirá para determinados valores de $\xi$, dependendo do sinal do termo cúbico em (2.3). Suponha, por exemplo, o sistema

$$
\left\{\begin{array}{l}
\dot{\rho}=\rho\left(\xi-\rho^{2}\right) \\
\dot{\theta}=1
\end{array} .\right.
$$

Então, para $\xi>0, \rho(\xi)=\sqrt{\xi}$ é um ponto de equilíbrio que descreve uma órbita periódica
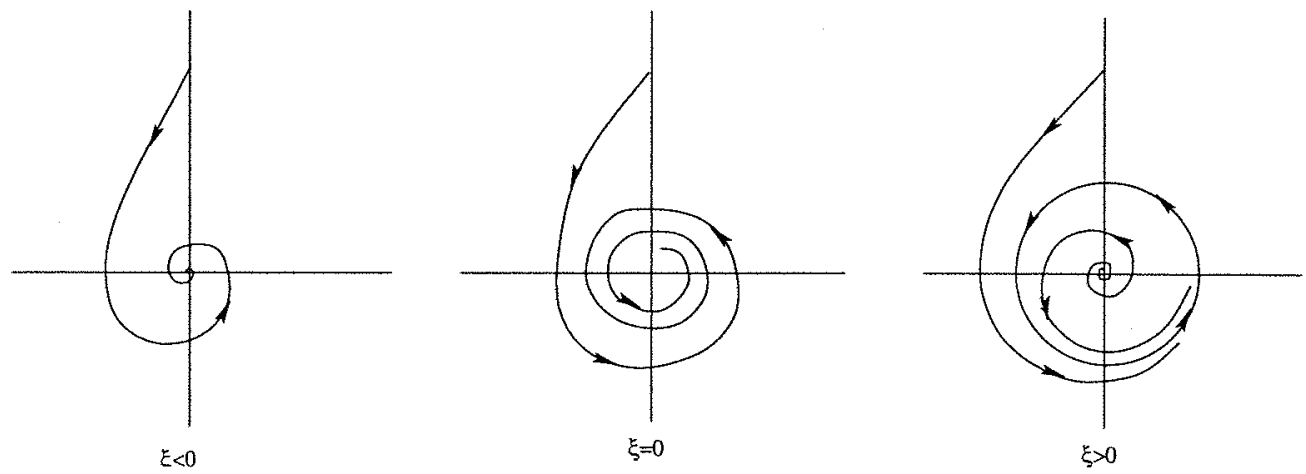

Figura 2.1: Retrato de fase da bifurcação de Hopf

circular com velocidade constante. Este sistema sempre tem um equilíbrio na origem que é um foco atrator se $\xi<0$, um foco repulsor para $\xi>0$ ou um foco atrator "fraco" (um equilíbrio não linear e topologicamente equivalente ao foco atrator), para o valor crítico $\xi=0$. Para $\xi>0$, a origem fica isolada por uma órbita fechada (ciclo limite) que é única e atratora. Este ciclo é uma circunferência de raio $\rho(\xi)=\sqrt{\xi}$. Todas as órbitas externas ou internas a este ciclo, com exceção da origem, tendem ao ciclo quando $t \rightarrow+\infty$, veja figura (2.1). Este fenômeno de geração de uma órbita periódica e a mudança de estabilidade do foco a 
partir de uma perturbação no parâmetro $\xi$ será chamado bifurcação de Andronov-Hopf ou simplesmente bifurcação de Hopf.

O mesmo sistema de (2.4), porém com sinal oposto nos termos não lineares,

$$
\left\{\begin{array}{l}
\dot{\rho}=\rho\left(\xi+\rho^{2}\right) \\
\dot{\theta}=1
\end{array}\right.
$$

pode ser analisado da mesma maneira. Teremos a bifurcação de Andronov-Hopf para $\xi=0$ mas, ao contrário de (2.4) o ciclo limite, que surgirá para $\xi<0$, é repulsor. Para valores de $\xi>0$ a origem é um foco repulsor e não possui ciclo limite, quando $\xi=0$ será um foco repulsor "fraco" (não linear) e para $\xi<0$ um foco atrator. Neste último caso teremos então o ciclo limite repulsor dado por uma órbita fechada cujo desenho será dado por uma circunferência centrada na origem de raio $\rho(\xi)=\sqrt{\xi}$. Todas as órbitas iniciando externa ou internamente ao ciclo, com exceção da origem, tendem a este ciclo quando $t \rightarrow-\infty$.

Definição 2.0.3 Os sistemas (2.1), ou equivalentemente, (2.2) e (2.3), serão denominados forma normal da bifurcação de Hopf.

A seguinte definição será usada na próxima seção, onde estudaremos a bifurcação de Hopf genérica:

Definição 2.0.4 Dois sistemas

$$
\begin{aligned}
& \dot{x}=f(x, \xi), x \in \mathbb{R}^{n}, \quad \xi \in \mathbb{R}^{m}, \\
& \dot{y}=g(y, \zeta), y \in \mathbb{R}^{n}, \quad \zeta \in \mathbb{R}^{m},
\end{aligned}
$$

são ditos localmente topologicamente equivalentes em torno da origem se existir uma aplicação $(x, \xi) \mapsto\left(h_{\xi}(x), k(\xi)\right)$, definida em uma vizinhança $V=U_{0} \times V_{0} d e(x, \xi)=(0,0)$, contida em $\mathbb{R}^{n} \times \mathbb{R}^{m}$, satisfazendo:

(i) $k: \mathbb{R}^{m} \rightarrow \mathbb{R}^{m}$ é um homeomorfismo definido em $V_{0}$;

(ii) $h_{\xi}: \mathbb{R}^{n} \rightarrow \mathbb{R}^{n}$ é um homeomorfismo para cada $\xi$, definido na vizinhança $U_{0}$ de $x=0, h_{0}(0)=0$, levando órbitas de (2.6) contidas em $U_{0}$ em órbitas de (2.7) em $h_{\xi}\left(U_{0}\right)$, preservando a direção do tempo. 


\subsection{Bifurcação de Hopf genérica}

Nesta seção encontraremos condições para que um sistema seja localmente topologicamente equivalente à forma normal, que acabamos de definir, para a bifurcação. Este resultado será obtido no teorema 2.1.1.

Considere o sistema

$$
\left(\begin{array}{c}
\dot{x_{1}} \\
\dot{x_{2}}
\end{array}\right)=\left(\begin{array}{cc}
\xi & -1 \\
1 & \xi
\end{array}\right)\left(\begin{array}{c}
x_{1} \\
x_{2}
\end{array}\right)-\left(x_{1}{ }^{2}+x_{2}{ }^{2}\right)\left(\begin{array}{c}
x_{1} \\
x_{2}
\end{array}\right)
$$

que, como definido no início do capítulo, representa a forma normal da bifurcação de Hopf cujo sinal dos termos cúbicos é negativo e, consequentemente, apresenta uma órbita periódica atratora.

Lema 2.1.1 O sistema

$$
\left(\begin{array}{c}
\dot{x_{1}} \\
\dot{x_{2}}
\end{array}\right)=\left(\begin{array}{cc}
\xi & -1 \\
1 & \xi
\end{array}\right)\left(\begin{array}{c}
x_{1} \\
x_{2}
\end{array}\right)-\left(x_{1}{ }^{2}+x_{2}{ }^{2}\right)\left(\begin{array}{c}
x_{1} \\
x_{2}
\end{array}\right)+O\left(\|x\|^{4}\right)
$$

onde $x=\left(x_{1}, x_{2}\right)^{t}$ e $O\left(\|x\|^{4}\right)$ representa os termos de ordem 4 e depende suavemente de $\xi$, é localmente topologicamente equivalente em torno da origem ao sistema (2.8).

\section{Demonstração 2.1.1}

PARTE 1 (Existência e Unicidade do ciclo).

Escrevendo (2.9) nas coordenadas polares $(\rho, \theta)$ obtemos:

$$
\left\{\begin{array}{l}
\dot{\rho}=\rho\left(\xi-\rho^{2}\right)+\Phi(\rho, \theta) \\
\dot{\theta}=1+\Psi(\rho, \theta)
\end{array}\right.
$$

onde $\Phi=O\left(|\rho|^{4}\right), \Psi=O\left(|\rho|^{3}\right)$, e não indicaremos a dependência em $\xi$ dessas funçôes para não complicarmos a notação. Uma órbita de (2.10) partindo de $(\rho, \theta)=\left(\rho_{0}, 0\right)$ tem a 


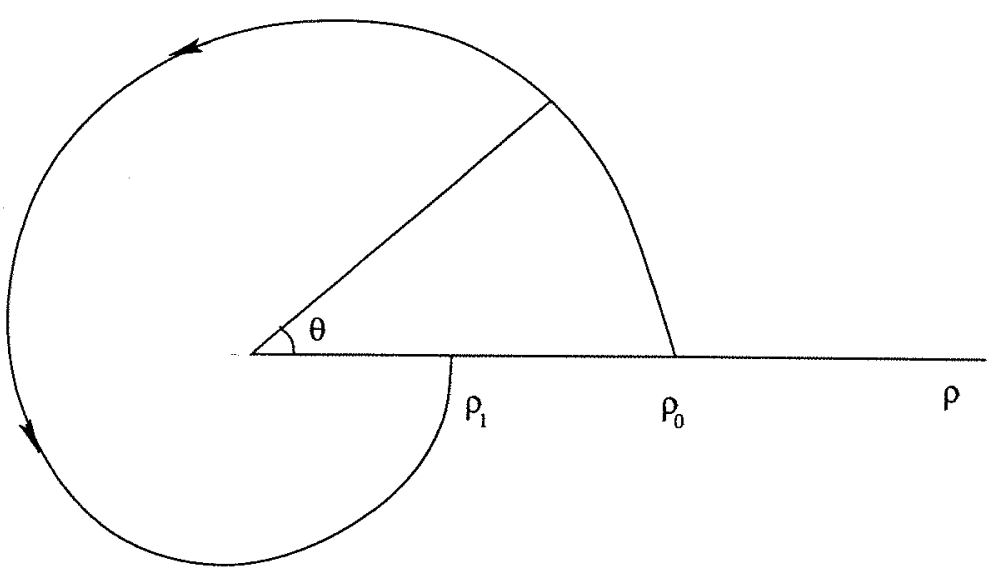

Figura 2.2: Mapeamento de Poincaré para a bifurcação de Hopf

seguinte representação, veja figura (2.2): $\rho=\rho\left(\theta ; \rho_{0}\right), \rho_{0}=\rho\left(0 ; \rho_{0}\right)$ com $\rho$ satisfazendo a equação

$$
\frac{d \rho}{d \theta}=\frac{\rho\left(\xi-\rho^{2}\right)+\Phi(\rho, \theta)}{1+\Psi(\rho, \theta)}=\rho\left(\xi-\rho^{2}\right)+R(\rho, \theta)
$$

onde $R=O\left(|\rho|^{4}\right)$. Note que a transformação de (2.10) para (2.11) é equivalente a uma reparametrização do tempo com $\dot{\theta}=1$, implicando que o tempo de retorno para o semi-eixo $\theta=0$ é o mesmo para todas as órbitas que partem desse eixo com $\rho_{0}>0$. Como $\rho(\theta ; 0) \equiv 0$, podemos escrever a expansão de Taylor para $\rho\left(\theta ; \rho_{0}\right)$,

$$
\rho=u_{1}(\theta) \rho_{0}+u_{2}(\theta) \rho_{0}^{2}+u_{3}(\theta) \rho_{0}^{3}+O\left(\left|\rho_{0}\right|^{4}\right)
$$

Substituindo (2.12) em (2.11) obtemos

$$
\begin{gathered}
\frac{d}{d \theta}\left(u_{1}(\theta) \rho_{0}+u_{2}(\theta) \rho_{0}{ }^{2}+u_{3}(\theta) \rho_{0}{ }^{3}+\ldots\right)= \\
=\left(u_{1}(\theta) \rho_{0}+u_{2}(\theta) \rho_{0}{ }^{2}+u_{3}(\theta) \rho_{0}{ }^{3}+\ldots\right)\left[\xi-\left(u_{1}(\theta) \rho_{0}+u_{2}(\theta) \rho_{0}{ }^{2}+u_{3}(\theta) \rho_{0}{ }^{3}+\ldots\right)^{2}\right]+R(\rho, \theta) \\
=u_{1}(\theta) \rho_{0} \xi+u_{2}(\theta) \rho_{0}{ }^{2} \xi+u_{3}(\theta) \rho_{0}{ }^{3} \xi-u_{1}{ }^{3}(\theta) \rho_{0}{ }^{3}+\ldots+R(\rho, \theta),
\end{gathered}
$$

de onde vem as seguintes equações diferenciais lineares resultantes das correspondentes potências de $\rho_{0}$

$$
\frac{d u_{1}}{d \theta}=u_{1} \xi, \frac{d u_{2}}{d \theta}=u_{2} \xi, \quad \frac{d u_{3}}{d \theta}=u_{3} \xi-u_{1}{ }^{3}
$$




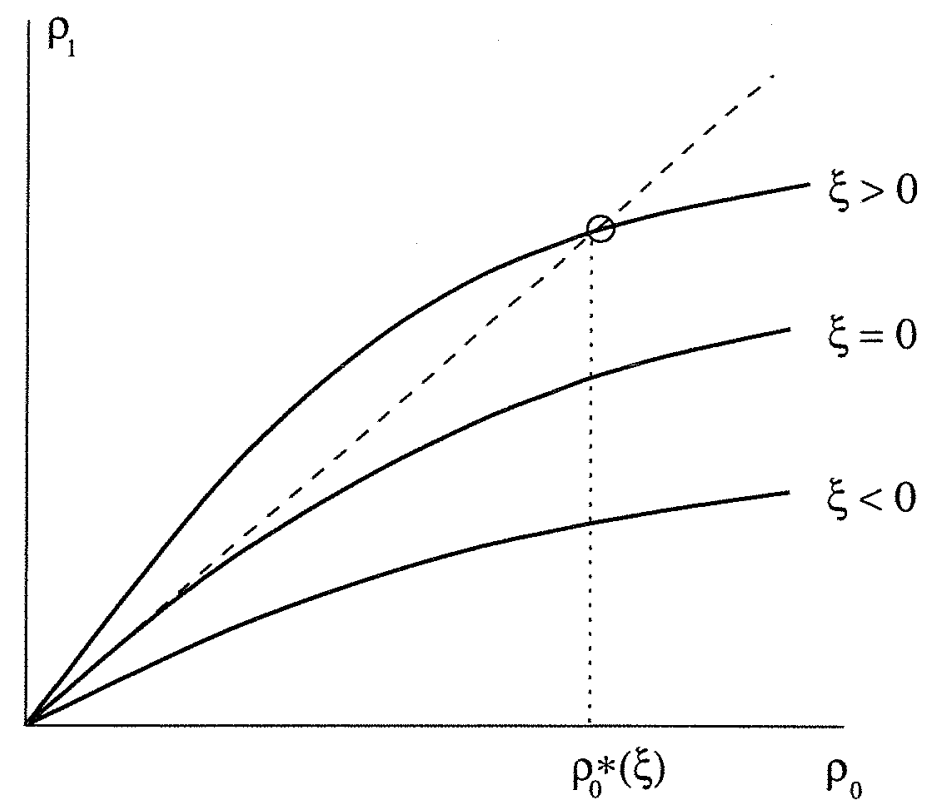

Figura 2.3: Ponto fixo do mapeamento de retorno

Como queremos, para $\theta=0, \rho=\rho_{0}$, estabelecemos as condições iniciais $u_{1}(0)=1, u_{2}(0)=$ $u_{3}(0)=0$, obtendo assim

$$
u_{1}(\theta)=e^{\xi \theta}, u_{2}(\theta) \equiv 0, u_{3}(\theta)=e^{\xi \theta} \frac{1-e^{2 \xi \theta}}{2 \xi} .
$$

Note que essas expressões são independentes de $R(\rho, \theta)$. Como na expressão de $u_{3}(2 \pi)$ vale a igualdade

$$
e^{2 \pi \xi} \frac{1-e^{2(2 \pi) \xi}}{2 \xi}=\frac{e^{2 \pi \xi}}{2 \xi}\left[1-\left(1+2(2 \pi) \xi+\frac{(2(2 \pi))^{2} \xi^{2}}{2 !}+\ldots\right)\right]=-e^{2 \pi \xi}[2 \pi+O(\xi)],
$$

podemos concluir que o mapeamento de retorno $\rho_{0} \mapsto \rho_{1}=\rho\left(2 \pi, \rho_{0}\right)$ tem a forma

$$
\rho_{1}=e^{2 \pi \xi} \rho_{0}-e^{2 \pi \xi}[2 \pi+O(\xi)] \rho_{0}^{3}+O\left(\rho_{0}^{4}\right)
$$

para todo $R=O\left(\rho^{4}\right)$. O mapeamento (2.13) pode ser facilmente analisado para $\rho_{0}$ e $|\xi|$ suficientemente pequenos. Existe uma vizinhança da origem onde esse mapeamento tem somente o ponto fixo trivial para pequenos valores de $\xi<0$ e um ponto fixo extra, $\rho_{0}^{*}=$ $\sqrt{\xi}+\ldots$, para pequenos valores de $\xi>0$, veja figura (2.3). Para verificar essa última afirmação, consideremos o mapeamento (2.13) escrito na forma:

$$
\rho_{1}=\rho_{0} \tilde{S}\left(\xi, \rho_{0}\right)
$$


onde

$$
\tilde{S}\left(\xi, \rho_{0}\right)=e^{2 \pi \xi}\left(1-[2 \pi+O(\xi)] \rho_{0}^{2}\right)+O\left(\rho_{0}^{3}\right) .
$$

Teremos, então, a equação dos pontos fixos, para $\rho_{0}>0$, dada por

$$
\begin{aligned}
& \tilde{S}\left(\xi, \rho_{0}\right)=1 \\
& \Leftrightarrow \quad e^{2 \pi \xi}\left(1-[2 \pi+O(\xi)] \rho_{0}^{2}\right)+O\left(\rho_{0}^{3}\right)=1 \\
& \Leftrightarrow \quad 1-[2 \pi+O(\xi)] \rho_{0}^{2}+e^{-2 \pi \xi} O\left(\rho_{0}^{3}\right)=e^{-2 \pi \xi} \\
& \Leftrightarrow 1-[2 \pi+O(\xi)] \rho_{0}^{2}+e^{-2 \pi \xi} O\left(\rho_{0}^{3}\right)-e^{-2 \pi \xi}=0 .
\end{aligned}
$$

Seja

$$
S\left(\xi, \rho_{0}\right)=1-[2 \pi+O(\xi)] \rho_{0}^{2}+e^{-2 \pi \xi} O\left(\rho_{0}{ }^{3}\right)-e^{-2 \pi \xi} .
$$

Aplicando o Teorema da Função Implícita na função $S\left(\xi, \rho_{0}\right)$, para $\left(\xi, \rho_{0}\right)=(0,0)$, comprovamos a afirmação. De fato $S(0,0)=0$ e $S_{\xi}(0,0)=2 \pi \neq 0$, o que nos permite escrever $\xi$ como função de $\rho_{0}$ numa vizinhança de $\rho_{0}=0$ e calcular

$$
\xi^{\prime}\left(\rho_{0}\right)=-\frac{S_{\rho_{0}}\left(\rho_{0}, \xi\left(\rho_{0}\right)\right)}{S_{\xi}\left(\rho_{0}, \xi\left(\rho_{0}\right)\right)}=\frac{2(2 \pi+O(\xi)) \rho_{0}+e^{-2 \pi \xi} O\left(\rho_{0}^{2}\right)}{(\ldots) \rho_{0}^{2}-2 \pi e^{-2 \pi \xi} O\left(\rho_{0}^{3}\right)+2 \pi e^{-2 \pi \xi}} .
$$

Portanto temos que

$$
\xi^{\prime}(0)=0, \quad \xi^{\prime \prime}(0)=2,
$$

implicando, pela expansão de Taylor ao redor de $\rho_{0}=0, \xi(0)=0$, que

$$
\xi\left(\rho_{0}\right)=\rho_{0}^{2}+\ldots
$$

que é uma função injetora no dominio $\rho_{0} \geq 0$.

A estabilidade dos pontos fixos também é obtida de (2.13). Derivando (2.14) com relação a $\rho_{0}$, obtemos:

$$
\frac{d \rho_{1}}{d \rho_{0}}=\tilde{S}\left(\xi, \rho_{0}\right)+\rho_{0} \tilde{S}_{\rho_{0}}\left(\xi, \rho_{0}\right)
$$

Para provarmos a estabilidade de $\rho_{0}^{*}$ basta mostrarmos que

$$
\frac{d \rho_{1}}{d \rho_{0}}\left(\rho_{0}^{*}\right)<1
$$

De fato, como $\tilde{S}\left(\xi, \rho_{0}\right)=1$ para $\rho_{0}=\rho_{0}^{*} ; \xi=\xi\left(\rho_{0}^{*}\right)$, resta vermos que $\rho_{0} \tilde{S}_{\rho_{0}}\left(\xi\left(\rho_{0}^{*}\right), \rho_{0}^{*}\right) \dot{e}$ negativo. Calculando $\rho_{0} \tilde{S}_{\rho_{0}}\left(\xi, \rho_{0}\right)=\rho_{0} \frac{\partial \tilde{S}}{\partial p_{0}}\left(\xi, \rho_{0}\right)$, obtemos:

$$
\rho_{0} \tilde{S}_{\rho_{0}}\left(\xi, \rho_{0}\right)=\rho_{0}{ }^{2}\left[-2 e^{2 \pi \xi}[2 \pi+O(\xi)]+O\left(\rho_{0}\right)\right],
$$


que, para pequenos valores de $\rho_{0}^{*}>0 ; \xi\left(\rho_{0}^{*}\right)>0$, satisfaz o esperado.

Levando em conta que o ponto fixo positivo do mapeamento corresponde a um ciclo limite do sistema, podemos concluir que o sistema (2.10), ou (2.9), com quaisquer termos $O\left(|\rho|^{4}\right)$, tem um único (e estável) ciclo limite bifurcando da origem quando $\xi>0$ como no sistema (2.8). Portanto, em outras palavras, os termos de ordem superior não afetam o surgimento do ciclo limite numa vizinhança de $\left(x_{1}, x_{2}\right)=(0,0)$ com $|\xi|$ suficientemente pequeno.

PARTE 2 (Construção do homeomorfismo).

Estabelecida a existência e unicidade do ciclo limite, indicaremos agora como proceder para se obter os homeomorfismos necessários e concluir a equivalência topológica dos retratos de fase.

Fixemos $\xi$ pequeno mas positivo. Ambos os sistemas (2.9) e (2.8) têm um ciclo limite em alguma vizinhança da origem. Assuma que já tenha sido realizada no sistema (2.9) a reparametrização do tempo, resultando num tempo de retorno constante $2 \pi$ (veja Parte 1 ). Além disso, aplicamos um escalonamento linear nas coordenadas do sistema (2.9) de modo que o ponto de interseç̧ão do ciclo e o semi-eixo horizontal seja $x_{1}=\sqrt{\xi}$.
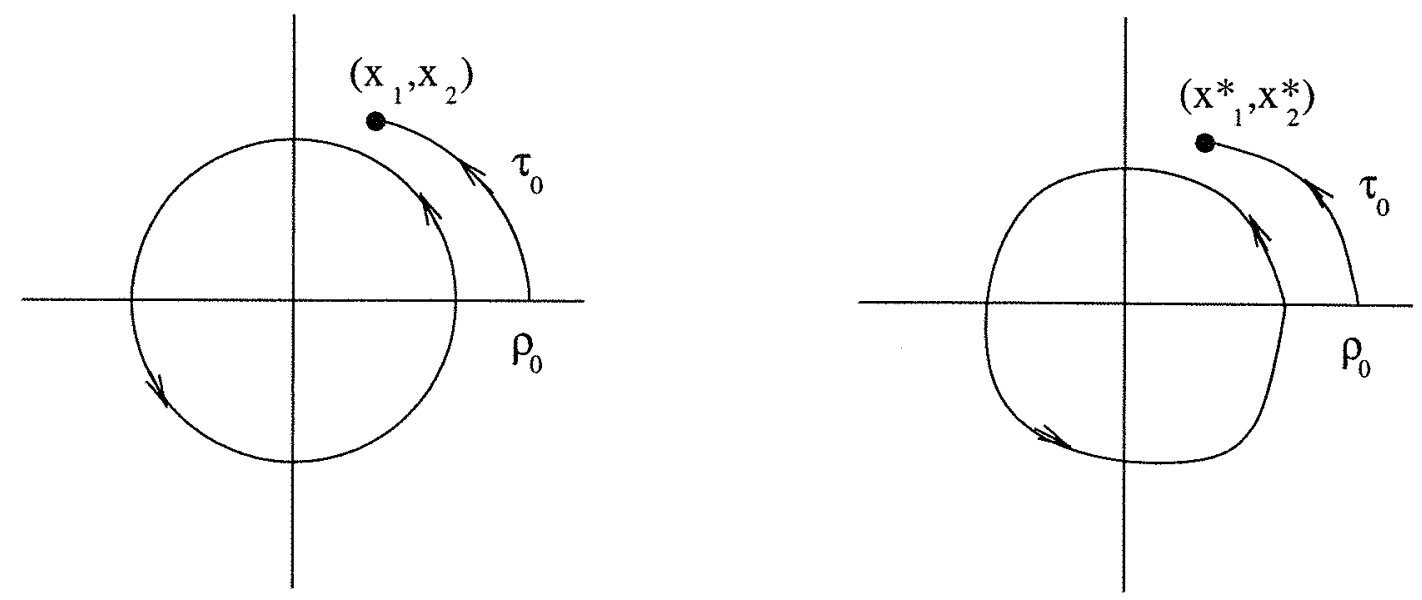

Figura 2.4: Construção do homeomorfismo próximo à bifurcação de Hopf

Defina o mapeamento $x \mapsto x^{*}$ do seguinte modo: Pegue o ponto $x=\left(x_{1}, x_{2}\right)$ e encontre valores $\left(\rho_{0}, \tau_{0}\right)$, onde $\tau_{0}$ é o tempo minimo que uma órbita do sistema (2.8) leva para alcançar 
o ponto $x$ partindo do semi-eixo horizontal com $\rho=\rho_{0}$. Agora, pegue o ponto deste eixo com $\rho=\rho_{0}$ e construa uma órbita do sistema (2.9) no intervalo $\left[0 ; \tau_{0}\right]$ partindo desse ponto. Denote o ponto resultante por $x^{*}=\left(x_{1}^{*}, x_{2}^{*}\right)$, veja figura (2.4). Assuma que $x^{*}=0$ para $x=0$.

O mapeamento construído é um homeomorfismo que, para $\xi>0$, leva órbitas do sistema (2.8), em alguma vizinhança da origem, em órbitas de (2.9), preservando a direção do tempo. $O$ caso $\xi<0$ pode ser considerado da mesma forma com uma nova mudança de coordenadas.

Considere o sistema

$$
\dot{x}=f(x, \xi), x=\left(x_{1}, x_{2}\right)^{t} \in \mathbb{R}^{2}, \xi \in \mathbb{R}
$$

com $f$ suave, tendo para $\xi=0$ o equilíbrio $x=0$ com autovalores $\lambda_{1,2}= \pm i \omega_{0}, \omega_{0}>0$. Pelo Teorema da Função Implícita, quando $\lambda=0$ não é um autovalor da matriz Jacobiana, o sistema tem um único equilíbrio $x_{0}(\xi)$ em alguma vizinhança da origem para todo $|\xi|$ suficientemente pequeno. Podemos, então, através de uma mudança de coordenadas, levar este equilíbrio para origem. Portanto vamos assumir sem perda de generalidade que $x=0$ é ponto de equilíbrio do sistema para $|\xi|$ suficientemente pequeno.

Então, o sistema pode ser escrito como

$$
\dot{x}=A(\xi) x+F(x, \xi)
$$

onde $F$ é uma função suave com componentes $F_{1,2}$ tendo expansão de Taylor em $x$ iniciando com termos quadráticos (no mínimo), $F=O\left(\|x\|^{2}\right)$. A matriz Jacobiana $A(\xi)$ possui dois autovalores

$$
\lambda_{1}(\xi)=\lambda(\xi), \lambda_{2}(\xi)=\bar{\lambda}(\xi)
$$

onde

$$
\lambda(\xi)=\gamma(\xi)+i \omega(\xi)
$$

e uma condição para a bifurcação de Hopf é:

$$
\gamma(0)=0, \omega(0)=\omega_{0}>0
$$


Seja $q(\xi) \in \mathbb{C}^{2}$ o autovetor complexo correspondente ao autovalor $\lambda(\xi)$,

$$
A(\xi) q(\xi)=\lambda(\xi) q(\xi)
$$

e seja $p(\xi) \in \mathbb{C}^{2}$ o autovetor da matriz transposta $A^{t}(\xi)$ correspondente ao autovalor $\bar{\lambda}(\xi)$,

$$
A^{t}(\xi) p(\xi)=\bar{\lambda}(\xi) p(\xi)
$$

É sempre possível normalizar $p$ com respeito a $q$ :

$$
<p(\xi), q(\xi)>=1
$$

onde $\langle p, q\rangle=\overline{p_{1}} q_{1}+\overline{p_{2}} q_{2}$ é o produto escalar em $\mathbb{C}^{2}$. Qualquer vetor $x \in \mathbb{R}^{2}$ pode ser representado unicamente para todo $\xi$ pequeno como

$$
x=z q(\xi)+\bar{z} \bar{q}(\xi)
$$

para algum complexo $z$. Temos então a seguinte fórmula explícita para se determinar $z$ :

$$
z=<p(\xi), x>
$$

Para verificar esta fórmula notemos que:

$$
\begin{gathered}
<p, x>=<p, z q+\bar{z} \bar{q}>=<p, z q>+<p, \bar{z} \bar{q}> \\
\Rightarrow<p, x>=z<p, q>+\bar{z}<p, \bar{q}>.
\end{gathered}
$$

Como $\langle p, q\rangle=1$ basta vermos que $\langle p, \bar{q}\rangle=0$. De fato,

$$
\begin{gathered}
<p, \bar{q}>=<p, \frac{1}{\bar{\lambda}} A \bar{q}>=\frac{1}{\bar{\lambda}}<A^{t} p, \bar{q}>=\frac{\lambda}{\bar{\lambda}}<p, \bar{q}> \\
\Rightarrow\left(1-\frac{\lambda}{\bar{\lambda}}\right)<p, \bar{q}>=0 .
\end{gathered}
$$

Como $\lambda \neq \bar{\lambda}$, pois para $|\xi|$ suficientemente pequeno temos $\omega(\xi)>0$, concluímos que

$$
<p, \bar{q}>=0
$$


Lema 2.1.2 O sistema (2.15) pode ser escrito, para $|\xi|$ suficientemente pequeno, na forma:

$$
\dot{z}=\lambda(\xi) z+g(z, \ddot{z}, \xi)
$$

onde $g=O\left(|z|^{2}\right)$ é uma função suave de $(z, \bar{z}, \xi)$, dada por

$$
g(z, \bar{z}, \xi)=<p(\xi), F(z q(\xi)+\bar{z} \bar{q}(\xi), \xi)>
$$

Demonstração 2.1.2 De (2.16) temos que a variável complexa z satisfaz a equação

$$
\begin{aligned}
\dot{z} & =<p(\xi), \dot{x}> \\
& =<p, A x+F(x)> \\
& =<p, A x>+<p, F(x)> \\
& =<p, A(z q+\bar{z} \bar{q})>+<p, F(z q+\bar{z} \bar{q})> \\
& =<p, A(z q)>+<p, A(\bar{z} \bar{q})>+<p, F(z q+\bar{z} \bar{q})> \\
& =\lambda z<p, q>+\bar{\lambda} \bar{z}<p, \bar{q}>+<p, F(z q+\bar{z} \bar{q})> \\
& =\lambda(\xi) z+<p(\xi), F(z q(\xi)+\bar{z} \bar{q}(\xi), \xi)>,
\end{aligned}
$$

obtendo então a forma (2.17), como queríamos.

Escrevendo $g$ em série de Taylor nas duas variáveis complexas $(z$ e $\bar{z})$ temos:

$$
g(z, \bar{z}, \xi)=\sum_{k+l \geq 2} \frac{1}{k ! l !} g_{k l}(\xi) z^{k} \bar{z}^{l}
$$

onde

$$
g_{k l}(\xi)=\frac{\partial^{k+l}}{\partial z^{k} \partial \bar{z}^{l}}<p(\xi), F(z q(\xi)+\bar{z} \bar{q}(\xi), \xi)>\left.\right|_{z=0}
$$

para $k+l \geq 2, k, l=0,1, \ldots$.

Suponha que, para $\xi=0$, a função $F(x, \xi)$ de (2.15) seja representada na forma

$$
F(x, 0)=\frac{1}{2} B(x, x)+\frac{1}{6} C(x, x, x)+O\left(\|x\|^{4}\right)
$$

onde $B(x, y)$ e $C(x, y, u)$ são funções multilineares simétricas de $x, y, u \in \mathbb{R}^{2}$. Em coordenadas temos:

$$
B_{i}(x, y)=\left.\sum_{j, k=1}^{2} \frac{\partial^{2} F_{i}(\eta, 0)}{\partial \eta_{j} \partial \eta_{k}}\right|_{\eta=0} x_{j} y_{k}, \quad i=1,2
$$




$$
C_{i}(x, y, u)=\left.\sum_{j, k, l=1}^{2} \frac{\partial^{3} F_{i}(\eta, 0)}{\partial \eta_{j} \partial \eta_{k} \partial \eta_{l}}\right|_{\eta=0} x_{j} y_{k} u_{l}, \quad i=1,2
$$

Então,

$$
B(z q+\bar{z} \bar{q}, z q+\bar{z} \bar{q})=z^{2} B(q, q)+2 z \bar{z} B(q, \bar{q})+\bar{z}^{2} B(\bar{q}, \bar{q})
$$

onde $q=q(0), p=p(0)$, e os coeficientes de Taylor $g_{k l}, k+l=2$, dos termos quadráticos em $g(z, \bar{z}, 0)$ podem ser expressos, agora, pelas fórmulas:

$$
g_{20}=<p, B(q, q)>, g_{11}=<p, B(q, \bar{q})>, g_{02}=<p, B(\bar{q}, \bar{q})>
$$

e cálculos similares com $C$ nos dão

$$
g_{21}=<p, C(q, q, \bar{q})>
$$

Lema 2.1.3 A equação

$$
\dot{z}=\lambda z+\frac{g_{20}}{2} z^{2}+g_{11} z \bar{z}+\frac{g_{02}}{2} \bar{z}^{2}+O\left(|z|^{3}\right)
$$

onde $\lambda=\lambda(\xi)=\gamma(\xi)+i \omega(\xi), \gamma(0)=0, \omega(0)=\omega_{0}>0$, e $g_{i j}=g_{i j}(\xi)$, pode ser transformada, pela mudança de coordenada complexa

$$
z=w+\frac{h_{20}}{2} w^{2}+h_{11} w \bar{w}+\frac{h_{02}}{2} \bar{w}^{2}
$$

para $|\xi|$ suficientemente pequeno, na equação sem termos quadráticos:

$$
\dot{w}=\lambda w+O\left(|w|^{3}\right)
$$

Demonstração 2.1.3 A mudança de variável inversa é dada pela expressão:

$$
w=z-\frac{h_{20}}{2} z^{2}-h_{11} z \bar{z}-\frac{h_{02}}{2} \bar{z}^{2}+O\left(|z|^{3}\right) .
$$

Assim sendo,

$$
\begin{aligned}
\dot{w} & =\dot{z}-h_{20} z \dot{z}-h_{11}(\bar{z} \dot{z}+z \dot{\bar{z}})-h_{02} \bar{z} \dot{\bar{z}}+\ldots \\
& =\lambda z+\left(\frac{g_{20}}{2}-\lambda h_{20}\right) z^{2}+\left(g_{11}-\lambda h_{11}-\bar{\lambda} h_{11}\right) z \bar{z}+\left(\frac{g_{02}}{2}-\bar{\lambda} h_{02}\right) \bar{z}^{2}+\ldots \\
& =\lambda w+\frac{1}{2}\left(g_{20}-\lambda h_{20}\right) w^{2}+\left(g_{11}-\bar{\lambda} h_{11}\right) w \bar{w}+\frac{1}{2}\left(g_{02}-(2 \bar{\lambda}-\lambda) h_{02}\right) \bar{w}^{2}+O\left(|w|^{3}\right) .
\end{aligned}
$$


Escolhendo, então,

$$
h_{20}=\frac{g_{20}}{\lambda}, h_{11}=\frac{g_{11}}{\bar{\lambda}}, h_{02}=\frac{g_{02}}{2 \bar{\lambda}-\lambda},
$$

eliminamos os termos quadráticos de (2.18). Essas substituições são sempre possíveis pois, para $|\xi|$ suficientemente pequeno, os denominadores nunca se anulam, afinal $\lambda(0)=i \omega_{0}$ com $\omega_{0}>0$.

Lema 2.1.4 A equação

$$
\dot{z}=\lambda z+\frac{g_{30}}{6} z^{3}+\frac{g_{21}}{2} z^{2} \bar{z}+\frac{g_{12}}{2} z \bar{z}^{2}+\frac{g_{03}}{6} \bar{z}^{3}+O\left(|z|^{4}\right)
$$

onde $\lambda=\lambda(\xi)=\gamma(\xi)+i \omega(\xi), \gamma(0)=0, \omega(0)=\omega_{0}>0$, e $g_{i j}=g_{i j}(\xi)$, pode ser transformada, pela mudança de coordenada complexa

$$
z=w+\frac{h_{30}}{6} w^{3}+\frac{h_{21}}{2} w^{2} \bar{w}+\frac{h_{12}}{2} w \bar{w}^{2}+\frac{h_{03}}{6} \bar{w}^{3}
$$

para $|\xi|$ suficientemente pequeno, na equação com somente um termo cúbico:

$$
\dot{w}=\lambda w+c_{1} w^{2} \tilde{w}+O\left(|w|^{4}\right)
$$

onde $c_{1}=c_{1}(\xi)$.

Demonstração 2.1.4 A transformação inversa é

$$
w=z-\frac{h_{30}}{6} z^{3}-\frac{h_{21}}{2} z^{2} \bar{z}-\frac{h_{12}}{2} z \bar{z}^{2}-\frac{h_{03}}{6} \bar{z}^{3}+O\left(|z|^{4}\right) .
$$

Temos então,

$$
\begin{aligned}
\dot{w}= & \dot{z}-\frac{h_{30}}{2} z^{2} \dot{z}-\frac{h_{21}}{2}\left(2 z \bar{z} \dot{z}+z^{2} \dot{\bar{z}}\right)-\frac{h_{12}}{2}\left(\bar{z}^{2} \dot{z}+2 z \bar{z} \dot{\bar{z}}\right)-\frac{h_{03}}{2} \bar{z}^{2} \dot{\bar{z}}+\ldots \\
= & \lambda z+\left(\frac{g_{30}}{6}-\frac{\lambda h_{30}}{2}\right) z^{3}+\left(\frac{g_{21}}{2}-\lambda h_{21}-\frac{\bar{\lambda} h_{21}}{2}\right) z^{2} \bar{z}+\left(\frac{g_{12}}{2}-\frac{\lambda h_{12}}{2}-\bar{\lambda} h_{12}\right) z \bar{z}^{2} \\
& +\left(\frac{g_{03}}{6}-\frac{\bar{\lambda} h_{03}}{2}\right) \bar{z}^{3}+\ldots \\
= & \lambda w+\frac{1}{6}\left(g_{30}-2 \lambda h_{30}\right) w^{3}+\frac{1}{2}\left(g_{21}-(\lambda+\bar{\lambda}) h_{21}\right) w^{2} \bar{w}+\frac{1}{2}\left(g_{12}-2 \bar{\lambda} h_{12}\right) w \bar{w}^{2} \\
& +\frac{1}{6}\left(g_{03}+(\lambda-3 \bar{\lambda}) h_{03}\right) \bar{w}^{3}+O\left(|w|^{4}\right) .
\end{aligned}
$$

Fazendo, portanto,

$$
h_{30}=\frac{g_{30}}{2 \lambda}, h_{12}=\frac{g_{12}}{2 \bar{\lambda}}, h_{03}=\frac{g_{03}}{3 \bar{\lambda}-\lambda},
$$


eliminamos todos os termos cúbicos com exceção do termo $w^{2} \bar{w}$, que será tratado separadamente. As substituições são válidas pois os denominadores envolvidos são diferentes de zero para todo $|\xi|$ suficientemente pequeno.

Uma tentativa de eliminar o termo $w^{2} \bar{w}$ seria escolher

$$
h_{21}=\frac{g_{21}}{\lambda+\bar{\lambda}}
$$

Isso é possivel para $\xi \neq 0$ pequeno, mas quando $\xi=0$ o denominador se anula, pois $\lambda(0)+$ $\bar{\lambda}(0)=i \omega_{0}-i \omega_{0}=0$. Para obtermos então uma transformação que dependa suavemente de $\xi$, escolhemos $h_{21}=0$, no que resulta:

$$
c_{1}=\frac{g_{21}}{2}
$$

O termo $w^{2} \bar{w}$ é chamado de termo ressonante. Note que o seu coeficiente é o mesmo coeficiente do termo cúbico $z^{2} \bar{z}$ na equação $(2.19)$.

Lema 2.1.5 A equação

$$
\dot{z}=\lambda z+\sum_{2 \leq k+l \leq 3} \frac{1}{k ! l !} g_{k l} z^{k} \bar{z}^{l}+O\left(|z|^{4}\right),
$$

onde $\lambda=\lambda(\xi)=\gamma(\xi)+i \omega(\xi), \gamma(0)=0, \omega(0)=\omega_{0}>0$, e $g_{i j}=g_{i j}(\xi)$, pode ser transformada, pela mudança de coordenada complexa

$$
z=w+\frac{h_{20}}{2} w^{2}+h_{11} w \bar{w}+\frac{h_{02}}{2} \bar{w}^{2}+\frac{h_{30}}{6} w^{3}+\frac{h_{12}}{2} w \bar{w}^{2}+\frac{h_{03}}{6} \bar{w}^{3},
$$

para $|\xi|$ suficientemente pequeno, na equação com apenas um termo cúbico:

$$
\dot{w}=\lambda w+c_{1} w^{2} \bar{w}+O\left(|w|^{4}\right)
$$

$\operatorname{com} c_{1}=c_{1}(\xi)$

Demonstração 2.1.5 Claramente os lemas 2.1.3 e 2.1.4 aplicam-se aqui e executam o serviço. Primeiramente a transformação

$$
z=w+\frac{h_{20}}{2} w^{2}+h_{11} w \bar{w}+\frac{h_{02}}{2} \bar{w}^{2}
$$


com

$$
h_{20}=\frac{g_{20}}{\lambda}, h_{11}=\frac{g_{11}}{\bar{\lambda}}, h_{02}=\frac{g_{02}}{2 \bar{\lambda}-\lambda},
$$

aniquila os termos quadráticos como vimos no lema 2.1.3, mas lembremos que ela também altera os coeficientes dos termos cúbicos. O coeficiente $\frac{1}{2} g_{21}$ do termo $z^{2} \bar{z}$ na equação (2.20) foi, portanto, modificado pela transformação (2.2Q). Representemos, então, o coeficiente de $w^{2} \bar{w}$ (termo ressonante) por $\frac{1}{2} g_{21}^{*}$. Quando aplicarmos a transformação do lema 2.1 .4 eliminaremos todos os termos cúbicos com exceção do termo ressonante. O coeficiente deste termo continuará sendo $\frac{1}{2} g_{21}^{*}$, como vimos no lema 2.1 .4 .

Necessitamos, agora, computar o coeficiente $c_{1}$ em termos da equação (2.20). $O$ valor de $c_{1}$ será dado pelo novo coeficiente $\frac{1}{2} g_{21}^{*}$ do termo $w^{2} \bar{w}$ após a transformação quadrática (2.22). Segue então o lema:

Lema 2.1.6 O coeficiente $c_{1}(\xi)$, da equação (2.21), para $\xi=0$, é dado por

$$
c_{1}(0)=\frac{i}{2 \omega_{0}}\left(g_{20} g_{11}-2\left|g_{11}\right|^{2}-\frac{1}{3}\left|g_{02}\right|^{2}\right)+\frac{g_{21}}{2} \text {. }
$$

Demonstração 2.1.6 Diferenciando (2.22), obtemos:

$$
\dot{z}=\dot{w}+h_{20} w \dot{w}+h_{11}(w \dot{\bar{w}}+\bar{w} \dot{w})+h_{02} \bar{w} \dot{\bar{w}}
$$

Substituindo $\dot{w}$ e seu complexo conjugado $\dot{\bar{w}}$, usando (2.21), obtemos

$$
\dot{z}=\lambda w+\lambda h_{20} w^{2}+(\lambda+\bar{\lambda}) h_{11} w \bar{w}+\bar{\lambda} h_{02} \bar{w}^{2}+c_{1} w^{2} \bar{w}+\ldots
$$

Por outro lado, na equação (2.20),

$$
\dot{z}=\lambda z+\frac{1}{2} g_{20} z^{2}+g_{11} z \bar{z}+\frac{1}{2} g_{02} \bar{z}^{2}+\frac{1}{6} g_{30} z^{3}+\frac{1}{2} g_{21} z^{2} \bar{z}+\frac{1}{2} g_{12} z \bar{z}^{2}+\frac{1}{6} g_{03} \bar{z}^{3}+\ldots
$$

se substituirmos $z$ e $\bar{z}$, dados por (2.2Q), escrevendo apenas os termos que nos interessam, temos

$$
\begin{aligned}
\dot{z}= & \lambda w+\frac{1}{2}\left(\lambda h_{20}+g_{20}\right) w^{2}+\left(\lambda h_{11}+g_{11}\right) w \bar{w}+\frac{1}{2}\left(\lambda h_{02}+g_{02}\right) \bar{w}^{2}+ \\
& \left(g_{20} h_{11}+g_{11}\left(\frac{h_{20}}{2}+\bar{h}_{11}\right)+\frac{g_{02} \bar{h}_{02}}{2}+\frac{g_{21}}{2}\right) w^{2} \bar{w}+\ldots
\end{aligned}
$$

Comparando, então, os coeficientes do termo $w^{2} \bar{w}$ nas duas equações obtidas, utilizando os valores encontrados para $h_{20}, h_{11}$ e $h_{02}$,

$$
h_{20}=\frac{g_{20}}{\lambda}, h_{11}=\frac{g_{11}}{\bar{\lambda}}, h_{02}=\frac{g_{02}}{2 \bar{\lambda}-\lambda},
$$


temos:

$$
\begin{aligned}
c_{1} & =g_{20} \frac{g_{11}}{\bar{\lambda}}+g_{11}\left(\frac{g_{20}}{2 \lambda}+\frac{\bar{g}_{11}}{\lambda}\right)+\frac{g_{02} \bar{g}_{02}}{2(2 \lambda-\bar{\lambda})}+\frac{g_{21}}{2} \\
\Rightarrow \quad c_{1} & =\frac{g_{20} g_{11}(2 \lambda+\bar{\lambda})}{2|\lambda|^{2}}+\frac{\left|g_{11}\right|^{2}}{\lambda}+\frac{\left|g_{02}\right|^{2}}{2(2 \lambda-\lambda)}+\frac{g_{21}}{2} .
\end{aligned}
$$

Essa fórmula nos dá a dependência de $c_{1}$ em relação a $\xi$, lembrando que $\lambda$ e $g_{i j}$ são funções suaves do parâmetro. No valor da bifurcação $\xi=0$, a última equação se reduz a

$$
c_{1}(0)=\frac{g_{20} g_{11}\left(2 i \omega_{0}-i \omega_{0}\right)}{2 \omega_{0}^{2}}+\frac{\left|g_{11}\right|^{2}}{i \omega_{0}}+\frac{\left|g_{02}\right|^{2}}{2\left(2 i \omega_{0}+i \omega_{0}\right)}+\frac{g_{21}}{2}
$$

concluido, finalmente, o resultado

$$
c_{1}(0)=\frac{i}{2 \omega_{0}}\left(g_{20} g_{11}-2\left|g_{11}\right|^{2}-\frac{1}{3}\left|g_{02}\right|^{2}\right)+\frac{g_{21}}{2} .
$$

Lema 2.1.7 Considere a equação

$$
\frac{d w}{d t}=(\gamma(\xi)+i \omega(\xi)) w+c_{1}(\xi) w|w|^{2}+O\left(|w|^{4}\right)
$$

onde $\gamma(0)=0$ e $\omega(0)=\omega_{0}>0$.

Suponha $\gamma^{\prime}(0) \neq 0$ e Re $c_{1}(0) \neq 0$. Então a equação acima poderá ser transformada, por mudanças de coordenadas, na equação:

$$
\frac{d u}{d \theta}=(\chi+i) u+s u|u|^{2}+O\left(|u|^{4}\right)
$$

onde u é a nova coordenada complexa, $\theta$ e $\chi$ são, respectivamente, os novos tempo e parâmetro es $=$ sinal Re $c_{1}(0)= \pm 1$.

Demonstração 2.1.7 Introduzindo o novo tempo $\tau=\omega(\xi) t$, que preserva a direção pois $\omega(\xi)>0$ para todo $|\xi|$ suficientemente pequeno, obtemos:

$$
\begin{gathered}
\frac{d w}{d \tau}=\frac{\gamma(\xi)+i \omega(\xi)}{\omega(\xi)} w+\frac{c_{1}(\xi)}{\omega(\xi)} w|w|^{2}+O\left(|w|^{4}\right) \\
\Rightarrow \frac{d w}{d \tau}=(\chi+i) w+d_{1}(\chi) w|w|^{2}+O\left(|w|^{4}\right)
\end{gathered}
$$

onde

$$
\chi=\chi(\xi)=\frac{\gamma(\xi)}{\omega(\xi)}, d_{1}(\chi)=\frac{c_{1}(\xi(\chi))}{\omega(\xi(\chi))}
$$


Podemos considerar $\chi$ como um novo parâmetro porque

$$
\chi(0)=0, \chi^{\prime}(0)=\frac{\gamma^{\prime}(0)}{\omega(0)} \neq 0,
$$

e, portanto, o Teorema da Função Inversa nos garante a existência local e suave de $\xi$ como função de $\chi$.

Vamos agora reparametrizar o tempo ao longo das órbitas com a nova mudança de tempo $\theta=\theta(\tau, \chi)$, onde

$$
d \theta=\left(1+e_{1}(\chi)|w|^{2}\right) d \tau
$$

com $e_{1}(\chi)=I m d_{1}(\chi)$. Essa mudança é próxima da identidade numa pequena vizinhança da origem. Usando esse novo valor de tempo definido, obtemos

$$
\frac{d w}{d \theta}=(\chi+i) w+l_{1}(\chi) w|w|^{2}+O\left(|w|^{4}\right)
$$

onde $l_{1}(\chi)=\operatorname{Re} d_{1}(\chi)-\chi e_{1}(\chi)$ é real e

$$
l_{1}(0)=\frac{\operatorname{Re} c_{1}(0)}{\omega(0)}
$$

De fato,

$$
\begin{aligned}
\frac{d w}{d \theta}= & \frac{d w}{\left(1+e_{1}(\chi)|w|^{2}\right) d \tau}=(\chi+i) w+l_{1}(\chi) w|w|^{2}+\ldots \\
\Leftrightarrow \frac{d w}{d \tau} & =\left(1+e_{1}(\chi)|w|^{2}\right)\left[(\chi+i) w+l_{1}(\chi) w|w|^{2}+\ldots\right] \\
& =(\chi+i) w+\left[l_{1}(\chi)+e_{1}(\chi)(\chi+i)\right] w|w|^{2}+\ldots \\
& =(\chi+i) w+\left[\operatorname{Re} d_{1}-\chi e_{1}+\chi e_{1}+i e_{1}\right] w|w|^{2}+\ldots \\
& =(\chi+i) w+\left[\operatorname{Re} d_{1}+i I m d_{1}\right] w|w|^{2}+\ldots \\
& =(\chi+i) w+d_{1}(\chi) w|w|^{2}+\ldots
\end{aligned}
$$

Finalmente, introduzindo a nova variável complexa u:

$$
w=\frac{u}{\sqrt{\left|l_{1}(\chi)\right|}}
$$

que é possivel pois $\operatorname{Re} c_{1}(0) \neq 0$ e, portanto, $l_{1}(0) \neq 0$. A equação toma então a forma:

$$
\frac{1}{\sqrt{\left|l_{1}\right|}} \frac{d u}{d \theta}=(\chi+i) \frac{u}{\sqrt{\left|l_{1}\right|}}+l_{1} \frac{u}{\sqrt{\left|l_{1}\right|}}\left|\frac{u}{\sqrt{\left|l_{1}\right|}}\right|^{2}+\ldots
$$




$$
\Rightarrow \frac{d u}{d \theta}=(\chi+i) u+\frac{l_{1}(\chi)}{\left|l_{1}(\chi)\right|} u|u|^{2}+O\left(|u|^{4}\right)=(\chi+i) u+s u|u|^{2}+O\left(|u|^{4}\right),
$$

$\operatorname{com} s=\operatorname{sinal} l_{1}(0)=\operatorname{sinal} \operatorname{Re} c_{1}(0)$.

Definição 2.1.1 A função $l_{1}(\chi)$ é chamada de primeiro coeficiente de Lyapunov.

O que (2.25) nos diz é que o primeiro coeficiente de Lyapunov, para $\chi=0$, pode ser calculado pela fórmula

$$
l_{1}(0)=\frac{1}{2 \omega_{0}^{2}} \operatorname{Re}\left(i g_{20} g_{11}+\omega_{0} g_{21}\right)
$$

Isto significa que necessitamos somente das segunda e terceira derivadas no ponto de bifurcação para calcularmos $l_{1}(0)$.

Observação 2.1.1 O valor de $l_{1}(0)$ dependerá da normalização dos autovetores $q$ e $p$, en-

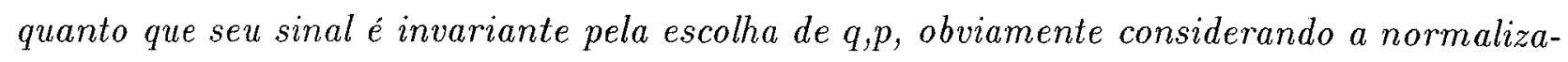
f̧ão $\langle p, q\rangle=1$.

Note que se a equação (2.24) com sinal $s=-1$ for escrita na sua forma real ela coincidirá com o sistema (2.9). Podemos agora resumir os resultados obtidos no seguinte teorema.

Teorema 2.1.1 Qualquer sistema em duas dimensões

$$
\frac{d x}{d t}=f(x, \xi), x \in \mathbb{R}^{2}, \xi \in \mathbb{R}
$$

com $f$ suave, tendo para todo $|\xi|$ suficientemente pequeno o equilíbrio $x=0$ com autovalores

$$
\lambda_{1,2}(\xi)=\gamma(\xi) \pm i \omega(\xi)
$$

onde $\gamma(0)=0, \omega(0)=\omega_{0}>0$, satisfazendo as seguintes condições:

(1) $l_{1}(0) \neq 0$ (não degenerescência);

(2) $\gamma^{\prime}(0) \neq 0$ (transversalidade),

é localmente topologicamente equivalente em torno da origem a uma das seguintes formas normais:

$$
\left(\begin{array}{l}
\dot{y_{1}} \\
\dot{y_{2}}
\end{array}\right)=\left(\begin{array}{cc}
\zeta & -1 \\
1 & \zeta
\end{array}\right)\left(\begin{array}{l}
y_{1} \\
y_{2}
\end{array}\right) \pm\left(y_{1}^{2}+y_{2}{ }^{2}\right)\left(\begin{array}{l}
y_{1} \\
y_{2}
\end{array}\right)
$$


Demonstração: Basta utilizar os lemas 2.1.3 a 2.1.7, para transformar o sistema (2.27) na equação (2.24) e então, usando o lema 2.1.1, concluímos o resultado.

Portanto, o que o Teorema 2.1.1 nos garante é que um sistema em duas dimensões que possui autovalores imaginários puros e satisfaz as condições (1) e (2) desse mesmo Teorema possui uma bifurcação de Hopf.

\subsection{Método da projeção}

Estudada a bifurcação de Hopf em sistemas de duas dimensões, nosso objetivo agora é apresentar um método para estudá-la em sistemas $n$-dimensionais. Tal método baseia-se em transformar o sistema, escrevendo-o em uma base formada pelos seus autovetores. Porém, somente autovetores correspondentes aos autovalores críticos (responsáveis pela bifurcação) são usados para se projetar o sistema e restringí-lo ao caso bidimensional.

Inicialmente faremos um breve resumo de alguns resultados da Âlgebra Linear que serão necessários para a seção.

Seja $A$ uma matriz quadrada e $\lambda$ um autovalor de $A$ com multiplicidade algébrica $m$, com $v_{1}, v_{2}, \ldots, v_{l}, 1 \leq l \leq m$, autovetores linearmente independentes correspondentes a $\lambda$. Para cada autovetor $v_{j}$, existe uma escolha maximal de vetores $w_{1}^{(j)}, w_{2}^{(j)}, \ldots, w_{k}^{(j)}$, onde $k=k(j) \in \mathbb{N}$, tal que:

$$
\begin{aligned}
A w_{1} & =\lambda w_{1}, \\
A w_{2} & =\lambda w_{2}+w_{1}, \\
\ldots & \\
A w_{k} & =\lambda w_{k}+w_{k-1} .
\end{aligned}
$$

Note que podemos escolher o vetor $w_{1}=w_{1}^{(j)}$ como sendo o próprio autovetor $v_{j}$.

Definição 2.2.1 Os vetores $w_{i}^{(j)}, i \geq 2$, são chamados autovetores generalizados de $A$, correspondentes ao autovalor $\lambda$.

Os autovetores generalizados $w_{1}^{(j)}, w_{2}^{(j)}, \ldots, w_{k_{j}}^{(j)}$ relativos a um autovalor múltiplo $\lambda$ são sempre linearmente independentes e, o subespaço

$$
X=\left\{x \in \mathbb{C}^{n}: x=\alpha_{1} w_{1}^{(j)}+\alpha_{2} w_{2}^{(j)}+\ldots+\alpha_{k} w_{k}^{(j)}, \alpha_{i} \in \mathbb{C}\right\}
$$


é $A$-invariante.

O estudo das formas normais de Jordan nos garante que o espaço $\mathbb{C}^{n}$ pode ser decomposto em subespaços $A$-invariantes correspondentes aos autovalores de $A$ e gerados pelos respectivos autovetores e autovetores generalizados. Esses subespaços são chamados de autoespaços generalizados de $A$. Se a matriz $A$ é real, esses subespaços $A$-invariantes do $\mathbb{R}^{n}$ serão gerados pelos autovetores e autovetores generalizados de $A$, correspondentes aos autovalores reais e às partes real e imaginária dos autovalores complexos com, por exemplo, parte imaginária positiva. Ver Kuznetsov [12] e Pontryagin [15].

Consideremos o sistema de equações diferenciais ordinárias

$$
\dot{x}=A x+F(x), x \in \mathbb{R}^{n},
$$

onde $F(x)=O\left(\|x\|^{2}\right)$ é uma função suave e $A$ corresponde à parte linear do sistema, com $x_{0}=0$ sendo ponto de equilíbrio não hiperbólico, com um par de autovalores puramente imaginários, $\lambda$ e $\bar{\lambda}$, onde $\lambda=i \omega_{0}, \omega_{0}>0, \omega_{0}$ real. Suponha que estes sejam os únicos autovalores de $A$ com parte real nula. Seja $q \in \mathbb{C}^{n}$ um autovetor correspondente a $\lambda$. Então

$$
A q=i \omega_{0} q, A \bar{q}=-i \omega_{0} \bar{q}
$$

Introduzimos agora o autovetor adjunto $p \in \mathbb{C}^{n}$ com a propriedade:

$$
A^{t} p=-i \omega_{0} p, A^{t} \bar{p}=i \omega_{0} \bar{p}
$$

e satisfazendo à normalização:

$$
<p, q>=1
$$

onde $\langle p, q\rangle=\sum_{i=1}^{n} \bar{p}_{i} q_{i}$ é o produto escalar padrão em $\mathbb{C}^{n}$ (linear com respeito ao segundo argumento). Considere o autoespaço real $T^{c}$, correspondente a $\lambda$ e $\bar{\lambda}$. $T^{c}$ tem dimensão dois e é gerado por $\{\operatorname{Re} q, \operatorname{Im} q\}$. O autoespaço real generalizado $T^{s u}$, correspondente a todos os outros autovalores de $A$, tem dimensão $(n-2)$.

Sempre podemos decompor $x \in \mathbb{R}^{n} \mathrm{em}$

$$
x=z q+\bar{z} \bar{q}+y_{s u}
$$

onde $z \in \mathbb{C}, z q+\bar{z} \bar{q} \in T^{c}$ e $y_{s u} \in T^{s u}$, uma vez que $T^{s u} \oplus T^{c}=\mathbb{R}^{n}$. 
Lema 2.2.1 Seja $y \in \mathbb{R}^{n} . y \in T^{s u}$ se e somente se $<p, y>=0$.

\section{Demonstração 2.2.1}

PARTE $1\left(y \in T^{s u} \Rightarrow\langle p, y\rangle=0\right)$.

Sejam $\mu_{1}, \mu_{2}, \ldots, \mu_{l}$, os autovalores reais de $A$ e $\eta_{1}, \bar{\eta}_{1} ; \eta_{2}, \bar{\eta}_{2} ; \ldots ; \eta_{k}, \bar{\eta}_{k}$, os autovalores complexos (não reais) de $A$, diferentes de $\lambda$ e $\bar{\lambda}$.

Seja $T_{\mu_{i}}$ o autoespaço generalizado correspondente ao autovalor $\mu_{i}$ e $T_{\eta_{j}, \bar{\eta}_{j}}$ o autoespaço real generalizado correspondente aos autovalores $\eta_{j}, \bar{\eta}_{j}$.

Temos, então, que

$$
T^{s u}=T_{\mu_{1}} \oplus T_{\mu_{2}} \oplus \ldots \oplus T_{\mu_{l}} \oplus T_{\eta_{1}, \bar{\eta}_{1}} \oplus T_{\eta_{2}, \bar{\eta}_{2}} \oplus \ldots \oplus T_{\eta_{k}, \bar{\eta}_{k}}
$$

Como $T_{\mu_{i}}$ são espaços generalizados, é fato que para cada $i$ existe um $N_{\mu_{i}} \in \mathbb{N}$, tal que, se $y \in T_{\mu_{i}}$, então $\left(A-\mu_{i} I\right)^{N_{\mu_{i}}} y=0$. Portanto,

$$
\begin{aligned}
0= & <p,\left(A-\mu_{i} I\right)^{N_{\mu_{i}}} y>=<\left(A^{t}-\bar{\mu}_{i} I\right)^{N_{\mu_{i}}} p, y>= \\
& =<\left(\bar{\lambda}-\bar{\mu}_{i}\right)^{N_{\mu_{i}}} p, y>=\left(\lambda-\mu_{i}\right)^{N_{\mu_{i}}}<p, y>
\end{aligned}
$$

e, como $\lambda \neq \mu_{i}$, temos que

$$
<p, y>=0
$$

Do mesmo modo, como $T_{\eta_{j}, \bar{\eta}_{j}}$ são espaços generalizados, para cada $j$ existe um $N_{\eta_{j}} \in \mathbb{N}$, tal que, se $y \in T_{\eta_{j}, \bar{\eta}_{j}}$, então $\left(A-\eta_{j} I\right)^{N_{\eta_{j}}}\left(A-\bar{\eta}_{j} I\right)^{N_{\eta_{j}}} y=0$. Portanto,

$$
\begin{gathered}
0=<p,\left(A-\eta_{j} I\right)^{N_{\eta_{j}}}\left(A-\bar{\eta}_{j} I\right)^{N_{\eta_{j}}} y>=<\left(A^{t}-\bar{\eta}_{j} I\right)^{N_{\eta_{j}}} p,\left(A-\bar{\eta}_{j} I\right)^{N_{\eta_{j}}} y>= \\
=<\left(A^{t}-\eta_{j} I\right)^{N_{\eta_{j}}}\left(A^{t}-\bar{\eta}_{j} I\right)^{N_{\eta_{j}}} p, y>=<\left(\bar{\lambda}-\eta_{j}\right)^{N_{\eta_{j}}}\left(\bar{\lambda}-\bar{\eta}_{j}\right)^{N_{\eta_{j}}} p, y>= \\
=\left(\lambda-\bar{\eta}_{j}\right)^{N_{\eta_{j}}}\left(\lambda-\eta_{j}\right)^{N_{\eta_{j}}}<p, y>.
\end{gathered}
$$

e, como $\lambda \neq \eta_{j}$ e $\lambda \neq \bar{\eta}_{j}$, temos que

$$
<p, y>=0
$$

Portanto, para qualquer $y \in T^{s u}$, como podemos escrever

$$
y=\sum_{i=1}^{l} y_{\mu_{i}}+\sum_{j=1}^{k} y_{\eta_{j}}
$$


com $y_{\mu_{i}} \in T_{\mu_{i}}$ para $i=1, \ldots, l$ e $y_{\eta_{j}} \in T_{\eta_{j}, \bar{n}_{j}}$ para $j=1, \ldots, k$, podemos concluir então que

$$
\begin{gathered}
<p, y>=<p, y_{\mu_{1}}+\ldots+y_{\mu_{l}}+y_{\eta_{1}}+\ldots+y_{\eta_{k}}>= \\
=<p, y_{\mu_{1}}>+\ldots+<p, y_{\mu_{l}}>+<p, y_{\eta_{1}}>+\ldots+<p, y_{\eta_{k}}>=0 .
\end{gathered}
$$

$\underline{\operatorname{PARTE} 2}\left(<p, y>=0, y \in \mathbb{R}^{n} \Rightarrow y \in T^{s u}\right)$.

Seja y qualquer, tal que $y \in T^{s u} \oplus T^{\mathcal{c}} \subset \mathbb{R}^{n}$. Portanto podemos escrever

$$
y=y_{s u}+y_{c}
$$

com $y_{s u} \in T^{s u}$ e $y_{c} \in T^{c}$. Como $T^{c}$ é gerado por $q, \bar{q}$ mas $y_{c} \in \mathbb{R}^{n}$,

$$
y_{c}=\alpha q+\bar{\alpha} \bar{q}
$$

$\operatorname{com} \alpha \in \mathbb{C}$, concluindo que

$$
y=y_{s u}+\alpha q+\bar{\alpha} \bar{q} .
$$

Queremos mostrar aqui que $y_{c}=0$, o que será feito mostrando que $\alpha=0$.

Da hipótese temos

$$
0=<p, y>=<p, y_{s u}+y_{c}>=<p, y_{s u}>+<p, y_{c}>
$$

Do inicio deste lema (Parte 1), temos que $<p, y_{s u}>=0$. Portanto

$$
\begin{array}{ll} 
& <p, y_{c}>=0 \\
\Rightarrow \quad & <p, \alpha q+\bar{\alpha} \bar{q}>=0 \\
\Rightarrow \quad & \alpha<p, q>+\bar{\alpha}<p, \bar{q}>=0 \\
\Rightarrow \quad & \alpha=0,
\end{array}
$$

pois $\langle p, q\rangle=1$ e $<p, \bar{q}>=0$. De fato,

$$
\begin{gathered}
<p, \bar{q}>=<p, \frac{1}{\bar{\lambda}} A \bar{q}>=\frac{1}{\bar{\lambda}}<A^{t} p, \bar{q}>=\frac{\lambda}{\bar{\lambda}}<p, \bar{q}> \\
\left(1-\frac{\lambda}{\bar{\lambda}}\right)<p, \bar{q}>=0
\end{gathered}
$$

Como $\lambda$ não é real, temos $\lambda \neq \bar{\lambda}$ e, portanto $<p, \bar{q}>=0$. 
Utilizando o lema anterior, podemos agora explicitar $z$ e $y$ com relação a $x$. Sendo $x=z q+\bar{z} \bar{q}+y \in \mathbb{R}^{n}, \operatorname{com} z q+\bar{z} \bar{q} \in T^{c}$ e $y \in T^{s u}$, vale que

$$
<p, x>=<p, z q+\bar{z} \bar{q}+y>=<p, z q>+<p, \bar{z} \bar{q}>+<p, y>
$$

Como $<p, y>=0$, pois $y \in T^{s u}$ (lema 2.2.1),

$$
<p, x>=<p, z q>+<p, \bar{z} \bar{q}>=z<p, q>+\bar{z}<p, \bar{q}>
$$

e, lembrando que $\langle p, q\rangle=1$ e $<p, \bar{q}\rangle=0$, como visto na demonstração do lema anterior (parte 2), concluímos que

$$
\left\{\begin{array}{l}
z=<p, x> \\
y=x-<p, x>q-<\bar{p}, x>\bar{q}
\end{array}\right.
$$

Lema 2.2.2 Nas coordenadas de (2.30), o sistema (2.28) tem a forma

$$
\left\{\begin{array}{l}
\dot{z}=i \omega_{0} z+<p, F(z q+\bar{z} \bar{q}+y)> \\
\dot{y}=A y+F(z q+\bar{z} \bar{q}+y)-<p, F(z q+\bar{z} \bar{q}+y)>q-<\bar{p}, F(z q+\bar{z} \bar{q}+y)>\bar{q}
\end{array} .\right.
$$

\section{Demonstração 2.2 .2}

$$
\begin{aligned}
\dot{z}=<p, \dot{x}> & =<p, A x+F(x)> \\
& =<p, A x>+<p, F(x)> \\
& =<p, A(z q+\bar{z} \bar{q}+y)>+<p, F(z q+\bar{z} \bar{q}+y)> \\
& =<p, z A q>+<p, \bar{z} A \bar{q}>+<p, A y>+<p, F(z q+\bar{z} \bar{q}+y)> \\
& =<p, z i \omega_{0} q>+<p, \bar{z}\left(-i \omega_{0} \bar{q}\right)>+<p, A y>+<p, F(z q+\bar{z} \bar{q}+y)>
\end{aligned}
$$

$<p, A y>=0$, pois $A y \in T^{s u}$ (lema 2.2.1). Portanto,

$$
\begin{aligned}
\dot{z} & =<p, z i \omega_{0} q>+<p, \bar{z}\left(-i \omega_{0} \bar{q}\right)>+<p, F(z q+\bar{z} \bar{q}+y)> \\
& =i \omega_{0} z<p, q>-i \omega_{0} \bar{z}<p, \bar{q}>+<p, F(z q+\bar{z} \bar{q}+y)> \\
& =i \omega_{0} z+<p, F(z q+\bar{z} \bar{q}+y)>
\end{aligned}
$$


$e$

$$
\begin{aligned}
\dot{y}= & \dot{x}-<p, \dot{x}>q-<\bar{p}, \dot{x}>\bar{q} \\
= & A x+F(x)-<p, A x+F(x)>q-<\bar{p}, A x+F(x)>\bar{q} \\
= & A x+F(x)-<p, A x>q-<p, F(x)>q-<\bar{p}, A x>\bar{q}-<\bar{p}, F(x)>\bar{q} \\
= & A(z q+\bar{z} \bar{q}+y)+F(x)-<p, A(z q+\bar{z} \bar{q}+y)>q-<p, F(x)>q \\
& \quad-<\bar{p}, A(z q+\bar{z} \bar{q}+y)>\bar{q}-<\bar{p}, F(x)>\bar{q} \\
= & A z q+A \bar{z} \bar{q}+A y+F(x)-<p, z A q>q-<p, \bar{z} A \bar{q}>q-<p, A y>q-<p, F(x)>q \\
& \quad-<\bar{p}, z A q>\bar{q}-<\bar{p}, \bar{z} A \bar{q}>\bar{q}-<\bar{p}, A y>\bar{q}-<\bar{p}, F(x)>\bar{q} \\
= & i \omega_{0} z q-i \omega_{0} \bar{z} \bar{q}+A y+F(x)-i \omega_{0} z<p, q>q+i \omega_{0} \bar{z}<p, \bar{q}>q-<p, A y>q \\
& \quad-<p, F(x)>q-i \omega_{0} z<\bar{p}, q>\bar{q}+i \omega_{0} \bar{z}<\bar{p}, \bar{q}>\bar{q}-<\bar{p}, A y>\bar{q}-<\bar{p}, F(x)>\bar{q} \\
= & i \omega_{0} z q-i \omega_{0} \bar{z} \bar{q}+A y+F(x)-i \omega_{0} z q-<p, F(x)>q+i \omega_{0} \bar{z} \bar{q}-<\bar{p}, F(x)>\bar{q} \\
= & +A y+F(z q+\bar{z} \bar{q}+y)-<p, F(z q+\bar{z} \bar{q}+y)>q-<\bar{p}, F(z q+\bar{z} \bar{q}+y)>\bar{q} .
\end{aligned}
$$

O sistema (2.31) pode ser escrito, usando a expansão de Taylor em $z, \bar{z}$ e $y$, na forma:

$$
\left\{\begin{array}{l}
\dot{z}=i \omega_{0} z+\frac{1}{2} G_{20} z^{2}+G_{11} z \bar{z}+\frac{1}{2} G_{02} \bar{z}^{2}+\frac{1}{2} G_{21} z^{2} \bar{z}+<G_{10}, y>z+<G_{01}, y>\bar{z}+\ldots \\
\dot{y}=A y+\frac{1}{2} H_{20} z^{2}+H_{11} z \bar{z}+\frac{1}{2} H_{02} \bar{z}^{2}+\ldots
\end{array}\right.
$$

onde $G_{20}, G_{11}, G_{02}, G_{21} \in \mathbb{C} ; G_{01}, G_{10}, H_{i j} \in \mathbb{C}^{n}$. Números complexos e vetores envolvendo (2.32) podem ser computados pelas fórmulas:

$$
\begin{gathered}
G_{i j}=\frac{\partial^{i+j}}{\partial z^{i} \partial \bar{z}^{j}}<p, F(z q+\bar{z} \bar{q})>\left.\right|_{z=0}, i+j \geq 2, \\
\bar{G}_{10, i}=\frac{\partial^{2}}{\partial y_{i} \partial z}<p, F(z q+\bar{z} \bar{q}+y)>\left.\right|_{z=0, y=0}, i=1,2, \ldots, n, \\
\bar{G}_{01, i}=\frac{\partial^{2}}{\partial y_{i} \partial \bar{z}}<p, F(z q+\bar{z} \bar{q}+y)>\left.\right|_{z=0, y=0}, i=1,2, \ldots, n, \\
H_{i j}=\left.\frac{\partial^{i+j}}{\partial z^{i} \partial \bar{z}^{j}} F(z q+\bar{z} \bar{q})\right|_{z=0}-G_{i j} q-\bar{G}_{j i} \bar{q}, i+j=2,
\end{gathered}
$$

onde

$$
<G, y>=\sum_{i=1}^{n} \bar{G}_{i} y_{i}
$$


Teorema 2.2.1 Teorema da Variedade Central Localmente, existe um conjunto invariante $W^{c}(0)$ de (2.28) que é tangente a $T^{c}$ em $x_{0}=0$. Tal conjunto é o gráfico de uma aplicação suave, cujas derivadas parciais de todas as ordens são unicamente determinadas.

Se $\psi^{t}$ denota o fluxo associado a (2.28), então existe uma vizinhança $U$ de $x_{0}=0$, tal que, se $\psi^{t} x \in U$ para todo $t \geq 0(t \leq 0)$, então $\psi^{t} x \rightarrow W^{c}(0)$ para $t \rightarrow+\infty(t \rightarrow-\infty)$. Ver Kuznetsov [12].

Definição 2.2.2 $W^{c}$ é chamado de variedade central.

Lema 2.2.3 O sistema (2.32), restrito a variedade central, fica dado pela equaçẫo

$$
\begin{aligned}
\dot{z}= & i \omega_{0} z+\frac{1}{2} G_{20} z^{2}+G_{11} z \bar{z}+\frac{1}{2} G_{02} \bar{z}^{2} \\
& +\frac{1}{2}\left(G_{21}-2<G_{10}, A^{-1} H_{11}>+<G_{01},\left(2 i \omega_{0} I-A\right)^{-1} H_{20}>\right) z^{2} \bar{z}+\ldots,
\end{aligned}
$$

onde I representa a matriz identidade.

Demonstração 2.2.3 A variedade central $W^{c}$ do sistema (2.32) pode ser localmente representada por um gráfico de uma função:

$$
W^{c}=\{(z, \bar{z}, y): y=V(z, \bar{z})\}
$$

Aqui $V: \mathbb{C}^{2} \rightarrow \mathbb{R}^{n}$ e satisfaz as duas propriedades de tangência de $W^{c}$,

$$
V(0,0)=0, \frac{d V}{d z}(0,0)=0, \frac{d V}{d \bar{z}}(0,0)=0,
$$

portanto $V(z, \bar{z})=O\left(|z|^{2}\right)$ e então

$$
y=V(z, \bar{z})=\frac{1}{2} w_{20} z^{2}+w_{11} z \bar{z}+\frac{1}{2} w_{02} \bar{z}^{2}+O\left(|z|^{3}\right),
$$

onde $\left\langle p, w_{i j}\right\rangle=0$. Derivando (2.34) obtemos:

$$
\dot{y}=w_{20} z \dot{z}+w_{11}(\bar{z} \dot{z}+z \dot{\bar{z}})+w_{02} \bar{z} \dot{\bar{z}}+\ldots
$$

Substituindo nessa equação as expressões de $\dot{z}$ e $\dot{\bar{z}}$, dadas pela primeira equação do sistema (2.32), obtemos:

$$
\begin{aligned}
\dot{y} & =w_{20} z\left(i \omega_{0} z+\ldots\right)+w_{11} \bar{z}\left(i \omega_{0} z+\ldots\right)+w_{11} z\left(-i \omega_{0} \bar{z}+\ldots\right)+w_{02} \bar{z}\left(-i \omega_{0} \bar{z}+\ldots\right)+\ldots \\
& =i \omega_{0} w_{20} z^{2}+i \omega_{0} w_{11} z \bar{z}-i \omega_{0} w_{11} z \bar{z}-i \omega_{0} w_{02} \bar{z}^{2}+\ldots \\
& =i \omega_{0} w_{20} z^{2}-i \omega_{0} w_{02} \bar{z}^{2}+\ldots
\end{aligned}
$$


Por outro lado, substituindo a expressão de y, dada em (2.34), na segunda equação do sistema (2.32), temos que

$$
\begin{aligned}
\dot{y} & =A\left(\frac{1}{2} w_{20} z^{2}+w_{11} z \bar{z}+\frac{1}{2} w_{02} \bar{z}^{2}+\ldots\right)+\frac{1}{2} H_{20} z^{2}+H_{11} z \bar{z}+\frac{1}{2} H_{02} \bar{z}^{2}+\ldots \\
& =\frac{1}{2}\left(A w_{20}+H_{20}\right) z^{2}+\left(A w_{11}+H_{11}\right) z \bar{z}+\frac{1}{2}\left(A w_{02}+H_{02}\right) \bar{z}^{2}+\ldots
\end{aligned}
$$

Comparando, então, as duas equações obtidas acima para $\dot{y}$, dos termos quadráticos obtemos:

$$
\left\{\begin{array}{rl}
\left(2 i \omega_{0} I-A\right) w_{20} & =H_{20} \\
-A w_{11} & =H_{11} \\
\left(-2 i \omega_{0} I-A\right) w_{02} & =H_{02}
\end{array} .\right.
$$

Estas equações tem solução única já que as matrizes que aparecem do lado esquerdo do

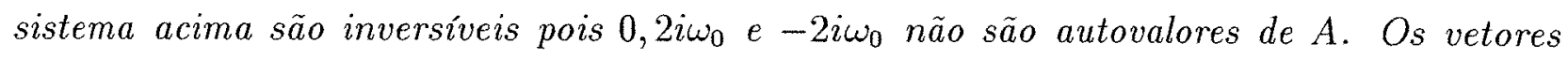
$w_{i j} \in \mathbb{C}^{n}$ podem, então, ser encontrados pelas equações lineares:

$$
\left\{\begin{array}{l}
w_{20}=\left(2 i \omega_{0} I-A\right)^{-1} H_{20} \\
w_{11}=-A^{-1} H_{11} \\
w_{02}=\left(-2 i \omega_{0} I-A\right)^{-1} H_{02}
\end{array} .\right.
$$

Calculados os coeficientes dos termos quadráticos na variedade central, devemos, agora, calcular o termo ressonante na primeira equação do sistema (2.32), restrita à variedade central. Note, então, que

$$
\begin{aligned}
<G, y> & =<G, \frac{1}{2} w_{20} z^{2}+w_{11} z \bar{z}+\frac{1}{2} w_{02} \bar{z}^{2}+\ldots> \\
& =\frac{1}{2}<G, w_{20}>z^{2}+<G, w_{11}>z \bar{z}+\frac{1}{2}<G, w_{02}>\bar{z}^{2}+\ldots
\end{aligned}
$$

Portanto, do produto $\left\langle G_{10}, y>z e<G_{01}, y>\bar{z}\right.$ do sistema (2.32), aparecem os termos $z^{2} \bar{z}$ com os seguintes coeficientes:

$$
\begin{aligned}
<G_{10}, y>z & =<G_{10}, w_{11}>z^{2} \bar{z}+\ldots \\
& =<G_{10},-A^{-1} H_{11}>z^{2} \bar{z}+\ldots \\
& =-<G_{10}, A^{-1} H_{11}>z^{2} \bar{z}+\ldots
\end{aligned}
$$

$e$

$$
\begin{aligned}
<G_{01}, y>\bar{z} & =\frac{1}{2}<G_{01}, w_{20}>z^{2} \bar{z}+\ldots \\
& =\frac{1}{2}<G_{01},\left(2 i \omega_{0} I-A\right)^{-1} H_{20}>z^{2} \bar{z}+\ldots
\end{aligned}
$$


Portanto,

$$
\begin{aligned}
\dot{z}= & i \omega_{0} z+\frac{1}{2} G_{20} z^{2}+G_{11} z \bar{z}+\frac{1}{2} G_{02} \bar{z}^{2} \\
& +\frac{1}{2} G_{21} z^{2} \bar{z}+<G_{10}, y>z+<G_{01}, y>\bar{z}+\ldots \\
= & i \omega_{0} z+\frac{1}{2} G_{20} z^{2}+G_{11} z \bar{z}+\frac{1}{2} G_{02} \bar{z}^{2} \\
& +\frac{1}{2} G_{21} z^{2} \bar{z}-<G_{10}, A^{-1} H_{11}>z^{2} \bar{z}+\frac{1}{2}<G_{01},\left(2 i \omega_{0} I-A\right)^{-1} H_{20}>z^{2} \bar{z}+\ldots
\end{aligned}
$$

O próximo lema exibirá um modo de calcularmos o primeiro coeficiente de Lyapunov $l_{1}$ utilizando apenas o autovetor $q$, respectivo ao autovalor $\omega_{0} i$ e o seu autovetor adjunto $p$.

\section{Lema 2.2.4 Condição de não degenerescência}

O primeiro coeficiente de Lyapunov do sistema (2.28) fica dado pela expressão:

$$
\begin{array}{r}
l_{1}(0)=\frac{1}{2 \omega_{0}} \operatorname{Re}\left[<p, C(q, q, \bar{q})>-2<p, B\left(q, A^{-1} B(q, \bar{q})\right)>\right. \\
\left.+<p, B\left(\bar{q},\left(2 i \omega_{0} I-A\right)^{-1} B(q, q)\right)>\right]
\end{array}
$$

Demonstração 2.2.4 Escrevemos $F(x)$ em termos de funções multilineares $B(x, y)$ e $C(x, y, z)$ :

$$
F(x)=\frac{1}{2} B(x, x)+\frac{1}{6} C(x, x, x)+O\left(\|x\|^{4}\right) .
$$

Podemos expressar então:

$$
<G_{10}, y>=<p, B(q, y)>, \quad<G_{01}, y>=<p, B(\bar{q}, y)>
$$

e a equação restrita (2.33) fica escrita na forma:

$$
\begin{aligned}
\dot{z}= & i \omega_{0} z+\frac{1}{2} G_{20} z^{2}+G_{11} z \bar{z}+\frac{1}{2} G_{02} \bar{z}^{2}+ \\
& \frac{1}{2}\left(G_{21}-2<p, B\left(q, A^{-1} H_{11}\right)>+<p, B\left(\bar{q},\left(2 i \omega_{0} I-A\right)^{-1} H_{20}\right)>\right) z^{2} \bar{z}+\ldots,
\end{aligned}
$$

onde

$$
\begin{gathered}
G_{20}=<p, B(q, q)>, G_{11}=<p, B(q, \bar{q})>, G_{02}=<p, B(\bar{q}, \bar{q})> \\
G_{21}=<p, C(q, q, \bar{q})>
\end{gathered}
$$

e

$$
\left\{\begin{array}{l}
H_{20}=B(q, q)-<p, B(q, q)>q-<\bar{p}, B(q, q)>\bar{q} \\
H_{11}=B(q, \bar{q})-<p, B(q, \bar{q})>q-<\bar{p}, B(q, \bar{q})>\bar{q}
\end{array} .\right.
$$


Substituindo de (2.38) a (2.40) em (2.37), e tomando

$$
\begin{aligned}
A^{-1} q & =\frac{1}{i \omega_{0}} q, A^{-1} \bar{q}=\frac{-1}{i \omega_{0}} \bar{q}, \\
\left(2 i \omega_{0} I-A\right)^{-1} q & =\frac{1}{i \omega_{0}} q,\left(2 i \omega_{0} I-A\right)^{-1} \bar{q}=\frac{1}{3 i \omega_{0}} \bar{q},
\end{aligned}
$$

transformamos (2.33) na equação

$$
\dot{z}=i \omega_{0} z+\frac{1}{2} g_{20} z^{2}+g_{11} z \bar{z}+\frac{1}{2} g_{02} \bar{z}^{2}+\frac{1}{2} g_{21} z^{2} \bar{z}+\ldots
$$

onde,

$$
g_{20}=<p, B(q, q)>, g_{11}=<p, B(q, \bar{q})>
$$

e

$$
\begin{aligned}
g_{21}= & <p, C(q, q, \bar{q})> \\
& -2<p, B\left(q, A^{-1} B(q, \bar{q})\right)>+<p, B\left(\bar{q},\left(2 i \omega_{0} I-A\right)^{-1} B(q, q)\right)> \\
& +\frac{1}{i \omega_{0}}<p, B(q, q)><p, B(q, \bar{q})> \\
& -\frac{2}{i \omega_{0}}|<p, B(q, \bar{q})>|^{2}-\frac{1}{3 i \omega_{0}}|<p, B(\bar{q}, \bar{q})>|^{2} .
\end{aligned}
$$

Note que os termos na última linha são imaginários puros enquanto os termos na terceira linha são dados pelo produto escalar $g_{20} g_{11}$. Aplicando então a fórmula

$$
l_{1}(0)=\frac{1}{2 \omega_{0}^{2}} \operatorname{Re}\left(i g_{20} g_{11}+\omega_{0} g_{21}\right)
$$

dada em (2.26), temos a seguinte expressão para o primeiro coeficiente de Lyapunov:

$$
\begin{array}{r}
l_{1}(0)=\frac{1}{2 \omega_{0}} \operatorname{Re}\left[<p, C(q, q, \bar{q})>-2<p, B\left(q, A^{-1} B(q, \bar{q})\right)>\right. \\
\left.+<p, B\left(\bar{q},\left(2 i \omega_{0} I-A\right)^{-1} B(q, q)\right)>\right] .
\end{array}
$$

Consideremos, agora, a família de sistemas de equações diferenciais a um parâmetro

$$
\dot{x}=A(\xi) x+F(x, \xi), \quad x \in \mathbb{R}^{n}, \xi \in \mathbb{R}
$$

onde $F(x, \xi)=O\left(\|x\|^{2}\right)$ é uma função suave de $x$, com sua expansão de Taylor iniciando com pelo menos termos quadráticos, e depende suavemente de $\xi$ e $A(\xi)$ corresponde à parte linear do sistema com um par de autovalores complexos

$$
\lambda_{1}(\xi)=\lambda(\xi), \quad \lambda_{2}(\xi)=\bar{\lambda}(\xi)
$$


onde

$$
\lambda(\xi)=\gamma(\xi)+i \omega(\xi)
$$

satisfazendo a condição de Hopf para $\xi=0$ :

$$
\gamma(0)=0, \omega(0)=\omega_{0}>0 .
$$

O próximo lema nos mostra como calcular $\gamma^{\prime}(0)$ em termos de $p$ e $q$.

\section{Lema 2.2.5 Condição de transversalidade}

Considere o sistema (2.41), cuja matriz Jacobiana $A(\xi)$ possui um par de autovalores puramente imaginários para $\xi=0, \lambda_{1,2}(\xi)=\gamma(\xi) \pm i \omega(\xi), \gamma(0)=0, \omega(0)=\omega_{0}>0$. Então,

$$
\gamma^{\prime}(0)=R e<p, A^{\prime}(0) q>
$$

onde $p, q \in \mathbb{C}^{n}$ satisfazem

$$
A(0) q=i \omega_{0} q, \quad A^{t}(0) p=-i \omega_{0} p, \quad<p, q>=1 .
$$

Demonstração 2.2.5 Derivando ambos os lados da equação

$$
A(\xi) q(\xi)=\lambda(\xi) q(\xi)
$$

com relação a $\xi$ obtemos

$$
A^{\prime}(\xi) q(\xi)+A(\xi) q^{\prime}(\xi)=\lambda^{\prime}(\xi) q(\xi)+\lambda(\xi) q^{\prime}(\xi)
$$

Aplicando, agora, o produto escalar por $p$ em ambos os lados, temos

$$
\begin{gathered}
<p, A^{\prime} q+A q^{\prime}>=<p, \lambda^{\prime} q+\lambda q^{\prime}> \\
\Rightarrow<p, A^{\prime} q>+<p, A q^{\prime}>=<p, \lambda^{\prime} q>+<p, \lambda q^{\prime}> \\
\Rightarrow<p, A^{\prime} q>+<A^{t} p, q^{\prime}>=\lambda^{\prime}<p, q>+\lambda<p, q^{\prime}>.
\end{gathered}
$$

Para $\xi=0, A^{t} p=-i \omega_{0} p$, portanto

$$
\begin{gathered}
<p, A^{\prime}(0) q>+i \omega_{0}<p, q^{\prime}>=\left(\gamma^{\prime}(0)+i \omega^{\prime}(0)\right)<p, q>+i \omega_{0}<p, q^{\prime}> \\
\Rightarrow<p, A^{\prime}(0) q>=\left(\gamma^{\prime}(0)+i \omega^{\prime}(0)\right)<p, q>
\end{gathered}
$$

e, finalmente, como $<p, q>=1$,

$$
<p, A^{\prime}(0) q>=\gamma^{\prime}(0)+i \omega^{\prime}(0) .
$$




\section{Capítulo 3}

\section{Estudo Qualitativo do Modelo}

Neste capítulo nos interessaremos em estudar o modelo proposto no capítulo 1 com respeito aos seus pontos de equilíbrio, a dinâmica das populações e o surgimento de uma bifurcação de Hopf. Verificaremos em que condições a bifurcação poderá aparecer para, no capítulo 4, comprovarmos o seu surgimento em pelo menos alguns casos.

Consideremos o sistema

$$
\dot{x}=F(x)
$$

$\operatorname{com} F(x)$ suave, $x \in \mathbb{R}^{n}$. Seguem algumas definições que utilizaremos adiante:

Definição 3.0.3 Um ponto singular $x_{0}$ do sistema (3.1) é chamado de hiperbólico se todos os autovalores de $J\left(x_{0}\right)$ têm parte real diferente de zero, onde $J\left(x_{0}\right)=D F\left(x_{0}\right)$ representa a matriz Jacobiana de $F(x)$ no ponto $x_{0}$. Utilizaremos a notação hiperbólico $\mathrm{n}-\mathrm{p}$ para designar um ponto hiperbólico com $n$ autovalores com parte real negativa e $p$ autovalores com parte real positiva.

Definição 3.0.4 Um ponto singular hiperbólico $x_{0}$ do sistema (3.1) chama-se um atrator se todos os autovalores de $J\left(x_{0}\right)$ têm parte real negativa. Se todos os autovalores de um atrator forem reais ele será chamado de um nó atrator e se todos os autovalores forem complexos de um foco atrator.

Definição 3.0.5 Um ponto singular hiperbólico $x_{0}$ do sistema (3.1) chama-se um repulsor se todos os autovalores de $J\left(x_{0}\right)$ têm parte real positiva. Se todos os autovalores de um 
repulsor forem reais ele será chamado de um nó repulsor e se todos os autovalores forem complexos de um foco repulsor.

Definição 3.0.6 Um ponto singular hiperbólico $x_{0}$ do sistema (3.1) chama-se sela se todos os autovalores de $J\left(x_{0}\right)$ forem reais e pelo menos dois deles possuirem sinais opostos. Utilizaremos, entãa, a notação sela $\mathbf{n - p}$, para indicar uma sela com $n$ autovalores negativos e $p$ autovalores positivos.

Definição 3.0.7 O número de autovalores de $J\left(x_{0}\right)$ que têm parte real negativa chama-se índice de estabilidade do ponto $x_{0}$ e será representado pela letra $\sigma$.

Definição 3.0.8 O ponto singular $x_{0}$ é uma sela-nó de (3.1), com $x \in \mathbb{R}^{2}$, se $J\left(x_{0}\right)=$ $D F\left(x_{0}\right)$ tem um autovalor $\lambda_{1}=0$ e o outro $\lambda_{2} \neq 0$. Se $v_{1}$ e $v_{2}$ são os autovetores correspondentes a $\lambda_{1}$ e $\lambda_{2}$, respectivamente, então $D^{2} F\left(x_{0}\right)\left(v_{1}^{2}\right)=a_{1} v_{1}+a_{2} v_{2}$, com $a_{1} \neq 0$. Ver Sotomayor [18].

Relembramos aqui que todos os parâmetros, $\alpha, \beta, \mu, \phi, c, k, \alpha^{\prime}, \beta^{\prime}, \mu^{\prime}, \phi^{\prime}, c^{\prime}, k^{\prime}$, definidos na tabela (1.1), são positivos. Esse fato será utilizado no decorrer deste capítulo sem novas menções à respeito.

\subsection{Estudo das equações desacopladas}

Nesta seção investigaremos o modelo

$$
\left\{\begin{array}{l}
\frac{d P}{d t}=\phi\left(1-\frac{M}{c}\right) M-(\alpha+\beta) P-k P G \\
\frac{d M}{d t}=\alpha P-\mu M \\
\frac{d L}{d t}=\phi^{\prime}\left(1-\frac{G}{c^{\prime}}\right) G-\left(\alpha^{\prime}+\beta^{\prime}\right) L+k^{\prime} P G \\
\frac{d G}{d t}=\alpha^{\prime} L-\mu^{\prime} G
\end{array},\right.
$$

com as equações desacopladas $\left(k=k^{\prime}=0\right)$, para obtermos informações que nos auxiliem a, posteriormente, analisar este por completo.

O sistema desacoplado fica, então, dado por:

$$
\left\{\begin{array}{l}
\frac{d x}{d t}=\phi\left(1-\frac{y}{c}\right) y-(\alpha+\beta) x \\
\frac{d y}{d t}=\alpha x-\mu y
\end{array}\right.
$$


Lema 3.1.1 Sendo $R=\frac{\alpha \phi}{\mu(\alpha+\beta)}$, o sistema apresenta dois pontos de equilíbrio dados por

$$
\begin{aligned}
& \left(x_{1}, y_{1}\right)=(0,0) e \\
& \left(x_{2}, y_{2}\right)=\left(\frac{\mu c}{\alpha}\left(1-\frac{1}{R}\right), c\left(1-\frac{1}{R}\right)\right) .
\end{aligned}
$$

Demonstração 3.1.1 De fato, da segunda equação do sistema

$$
\left\{\begin{array}{l}
\phi\left(1-\frac{y}{c}\right) y-(\alpha+\beta) x=0 \\
\alpha x-\mu y=0
\end{array}\right.
$$

obtemos a relação

$$
x=\frac{\mu}{\alpha} y
$$

que, substituindo na primeira equação nos dá

$$
\begin{aligned}
& \phi\left(1-\frac{y}{c}\right) y-(\alpha+\beta) \frac{\mu}{\alpha} y=0 \\
& y\left[\phi\left(1-\frac{y}{c}\right)-(\alpha+\beta) \frac{\mu}{\alpha}\right]=0
\end{aligned}
$$

$e$, portanto, temos que

$$
y=0 \text { ou } y=c\left(1-\frac{\mu(\alpha+\beta)}{\alpha \phi}\right)
$$

De (3.4) temos:

$$
y=0 \Rightarrow x=0
$$

$e$

$$
y=c\left(1-\frac{\mu(\alpha+\beta)}{\alpha \phi}\right) \Rightarrow x=\frac{\mu c}{\alpha}\left(1-\frac{\mu(\alpha+\beta)}{\alpha \phi}\right) .
$$

Observação 3.1.1 Para que o ponto $\left(x_{2}, y_{2}\right)$, definido em (3.3), exista, no sentido biológico, é necessário que $R \geq 1$, caso contrário as populações ficariam determinadas por um número negativo de individuos.

Lema 3.1.2 A origem $(0,0)$ é:
(a) uma sela se $R>1$,
(b) um nó atrator se $R<1$,
(c) uma sela nó se $R=1$. 
Demonstração 3.1.2 Sendo $J(x, y)$ a matriz Jacobiana do sistema desacoplado (3.2), calculada no ponto $(x, y)$, temos:

$$
J(x, y)=\left(\begin{array}{cc}
-(\alpha+\beta) & \phi-\frac{2 \phi}{c} y \\
\alpha & -\mu
\end{array}\right)
$$

$e$, portanto,

$$
J\left(x_{1}, y_{1}\right)=J(0,0)=\left(\begin{array}{cc}
-(\alpha+\beta) & \phi \\
\alpha & -\mu
\end{array}\right)
$$

Analisando, agora, o traço e o determinante de $J(0,0)$, obtemos, respectivamente:

$$
\operatorname{tr} J(0,0)=-(\alpha+\beta+\mu)<0
$$

$e$

$$
\operatorname{det} J(0,0)=\mu(\alpha+\beta)-\alpha \phi
$$

Portanto os autovalores ficam dados pela fórmula:

$$
\lambda=\frac{1}{2}\left(t r \pm \sqrt{t r^{2}-4 d e t}\right)
$$

resultando $\mathrm{em}$,

$$
\begin{aligned}
& \lambda=-\frac{1}{2}(\alpha+\beta+\mu) \pm \frac{1}{2} \sqrt{(\alpha+\beta+\mu)^{2}-4[\mu(\alpha+\beta)-\alpha \phi]} \\
& \Rightarrow \lambda=-\frac{1}{2}(\alpha+\beta+\mu) \pm \frac{1}{2} \sqrt{(\alpha+\beta+\mu)^{2}+4 \alpha \phi\left[1-\frac{1}{R}\right]} .
\end{aligned}
$$

(a) se $R>1$ (i.é., $\mu(\alpha+\beta)<\alpha \phi) \Rightarrow \operatorname{det} J(0,0)<0 \Rightarrow$ sela.

(b) se $R<1$ (i.é., $\mu(\alpha+\beta)>\alpha \phi) \Rightarrow \operatorname{det} J(0,0)>0$. 
Como

$$
\begin{aligned}
\Delta=t r^{2}-4 \text { det } & =(\alpha+\beta+\mu)^{2}-4[\mu(\alpha+\beta)-\alpha \phi] \\
& =(\alpha+\beta-\mu)^{2}+4 \alpha \phi>0
\end{aligned}
$$

$\Rightarrow$ nó atrator.

(c) se $R=1, \lambda_{1}=0$ e $\lambda_{2}=-(\alpha+\beta+\mu)<0$.

Queremos $v_{1}$ tal que $J(0,0) v_{1}=\lambda_{1} v_{1}$, o que implica

$$
\left(\begin{array}{cc}
-(\alpha+\beta) & \phi \\
\alpha & -\mu
\end{array}\right)\left(\begin{array}{l}
x_{1} \\
x_{2}
\end{array}\right)=\left(\begin{array}{l}
0 \\
0
\end{array}\right)
$$

Lembrando que $R=1 \Rightarrow \phi=\frac{\mu(\alpha+\beta)}{\alpha}$, fazendo $x_{1}=\mu$ obtemos $x_{2}=\alpha$. Portanto,

$$
v_{1}=\left(\begin{array}{l}
\mu \\
\alpha
\end{array}\right)
$$

Queremos, agora, $v_{2}$ tal que $J(0,0) v_{2}=\lambda_{2} v_{2}$, o que implica

$$
\left(\begin{array}{cc}
\mu & \phi \\
\alpha & \alpha+\beta
\end{array}\right)\left(\begin{array}{l}
x_{1} \\
x_{2}
\end{array}\right)=\left(\begin{array}{l}
0 \\
0
\end{array}\right)
$$

Lembrando que $R=1 \Rightarrow \phi=\frac{\mu(\alpha+\beta)}{\alpha}$, fazendo $x_{1}=\phi$ obtemos $x_{2}=-\mu$. Portanto,

$$
v_{2}=\left(\begin{array}{c}
\phi \\
-\mu
\end{array}\right)
$$

Verifiquemos agora, conforme a definição 3.0.8, a relação $D^{2} F\left(x_{0}\right)\left(v_{1}^{2}\right)=a_{1} v_{1}+a_{2} v_{2}$. 
Primeiramente calculamos

$$
D F(x)\left(v_{1}\right)=\left(\begin{array}{cc}
-(\alpha+\beta) & \phi-\frac{2 \phi}{c} y \\
\alpha & -\mu
\end{array}\right)\left(\begin{array}{l}
\mu \\
\alpha
\end{array}\right)=\left(\begin{array}{c}
-(\alpha+\beta) \mu+\alpha \phi-\frac{2 \alpha \phi}{c} y \\
0
\end{array}\right) .
$$

Temos, então, que

$$
D^{2} F(x)\left(v_{1}\right)=\left(\begin{array}{cc}
0 & -2 \frac{\alpha \phi}{c} \\
0 & 0
\end{array}\right)
$$

Portanto,

$$
D^{2} F(x)\left(v_{1}^{2}\right)=\left(\begin{array}{cc}
0 & -2 \frac{\alpha \phi}{c} \\
0 & 0
\end{array}\right)\left(\begin{array}{l}
\mu \\
\alpha
\end{array}\right)=\left(\begin{array}{c}
-2 \frac{\alpha^{2} \phi}{c} \\
0
\end{array}\right)=D^{2} F\left(x_{0}\right)\left(v_{1}^{2}\right) .
$$

Desse modo, temos que

$$
\begin{gathered}
\left(\begin{array}{c}
-2 \frac{\alpha^{2} \phi}{c} \\
0
\end{array}\right)=a_{1}\left(\begin{array}{l}
\mu \\
\alpha
\end{array}\right)+a_{2}\left(\begin{array}{c}
\phi \\
-\mu
\end{array}\right) \\
\Rightarrow\left\{\begin{array}{l}
a_{1} \mu+a_{2} \phi=-2 \frac{\alpha^{2} \phi}{c} \\
a_{1} \alpha-a_{2} \mu=0
\end{array} .\right.
\end{gathered}
$$

Da segunda equação temos $a_{1}=a_{2} \frac{\mu}{\alpha}$ que, substituindo então na primeira, obtemos:

$$
\begin{gathered}
a_{2} \frac{\mu^{2}}{\alpha}+a_{2} \phi=-2 \frac{\alpha^{2} \phi}{c} \\
a_{2}\left(\frac{\mu^{2}}{\alpha}+\frac{\mu(\alpha+\beta)}{\alpha}\right)=-2 \frac{\alpha \mu(\alpha+\beta)}{c} \\
a_{2}\left(\frac{\mu(\alpha+\beta+\mu)}{\alpha}\right)=-2 \frac{\alpha \mu(\alpha+\beta)}{c} \\
a_{2}=-2 \frac{\alpha^{2}(\alpha+\beta)}{c(\alpha+\beta+\mu)}
\end{gathered}
$$

e, portanto, 


$$
a_{1}=a_{2} \frac{\mu}{\alpha}=-2 \frac{\alpha^{2}(\alpha+\beta)}{c(\alpha+\beta+\mu)} \frac{\mu}{\alpha}=-2 \frac{\alpha^{2} \phi}{c(\alpha+\beta+\mu)}<0 .
$$

Lema 3.1.3 O ponto $\left(x_{2}, y_{2}\right)=\left(\frac{\mu c}{\alpha}\left(1-\frac{1}{R}\right), c\left(1-\frac{1}{R}\right)\right)$ é

$\left(\mathbf{a}_{1}\right) u m$ foco atrator se $R>1$ e $\phi>\frac{1}{4 \alpha}\left[(\alpha+\beta+\mu)^{2}+4 \mu(\alpha+\beta)\right]$,

(a $\left.\mathbf{a}_{2}\right)$ um nó atrator se $R>1$ e $\phi \leq \frac{1}{4 \alpha}\left[(\alpha+\beta+\mu)^{2}+4 \mu(\alpha+\beta)\right]$,

(b) uma sela se $R<1$,

(c) uma sela-nó se $R=1$.

Demonstração 3.1.3 Calculando a matriz Jacobiana (3.5) no ponto $\left(x_{2}, y_{2}\right)$, dado em (3.3), obtemos:

$$
J\left(x_{2}, y_{2}\right)=\left(\begin{array}{cc}
-(\alpha+\beta) & \phi-\frac{2 \phi}{c} c\left(1-\frac{1}{R}\right) \\
\alpha & -\mu
\end{array}\right)
$$

e, portanto,

$$
J\left(x_{2}, y_{2}\right)=\left(\begin{array}{cc}
-(\alpha+\beta) & \phi\left(\frac{2}{R}-1\right) \\
\alpha & -\mu
\end{array}\right)
$$

De fato,

$$
\begin{gathered}
\operatorname{tr} J\left(x_{2}, y_{2}\right)=-(\alpha+\beta+\mu)<0 \\
\operatorname{det} J\left(x_{2}, y_{2}\right)=\mu(\alpha+\beta)-\alpha \phi\left(\frac{2}{R}-1\right) \\
\Rightarrow \frac{\operatorname{det} J\left(x_{2}, y_{2}\right)}{\alpha \phi}=\frac{\mu(\alpha+\beta)}{\alpha \phi}-\left(\frac{2}{R}-1\right) \\
\Rightarrow \frac{\operatorname{det} J\left(x_{2}, y_{2}\right)}{\alpha \phi}=\frac{1}{R}-\frac{2}{R}+1=1-\frac{1}{R} .
\end{gathered}
$$

Portanto, 
(a) se $R>1 \Rightarrow \operatorname{det} J\left(x_{2}, y_{2}\right)>0$.

Como

$$
\begin{aligned}
\Delta=t r^{2}-4 d e t & =(\alpha+\beta+\mu)^{2}-4\left[\mu(\alpha+\beta)-\alpha \phi\left(\frac{2}{R}-1\right)\right] \\
& =(\alpha+\beta+\mu)^{2}+4 \mu(\alpha+\beta)-4 \alpha \phi
\end{aligned}
$$

Temos que:

$\left(\mathbf{a}_{1}\right)$ se $\phi>\frac{1}{4 \alpha}\left[(\alpha+\beta+\mu)^{2}+4 \mu(\alpha+\beta)\right] \Rightarrow \Delta<0 \Rightarrow$ foco atrator.

$\left(\mathbf{a}_{2}\right)$ se $\phi \leq \frac{1}{4 \alpha}\left[(\alpha+\beta+\mu)^{2}+4 \mu(\alpha+\beta)\right] \Rightarrow \Delta \geq 0 \Rightarrow$ nó atrator.

(b) se $R<1 \Rightarrow \operatorname{det} J\left(x_{2}, y_{2}\right)<0 \Rightarrow$ sela.

(c) se $R=1 \Rightarrow\left(x_{2}, y_{2}\right)=(0,0)$, caso já estudado no lema 3.1.2.

A figura (3.1) representa o comportamento das partes real e imaginária dos dois autovalores em $\left(x_{1}, y_{1}\right)$ e dos dois autovalores em $\left(x_{2}, y_{2}\right)$ com relação a $R$ ou, mais especificamente, com relação a $\phi$. No eixo horizontal representamos a parte real de $\lambda$ e no eixo vertical a parte imaginária de $\lambda$. Na figura $\mathrm{B}$, os pontos $\left(x_{1}, y_{1}\right)$ e $\left(x_{2}, y_{2}\right)$ colapsam, caso em que temos o surgimento da sela-nó, e então os autovalores dos dois pontos coincidem. 


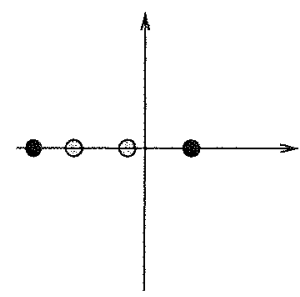

A

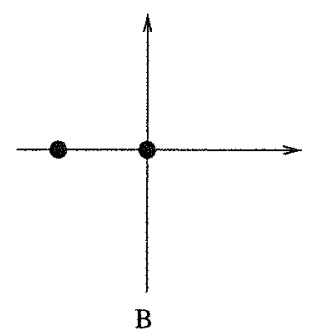

autovalores de $\left(x_{1}, y_{1}\right)$
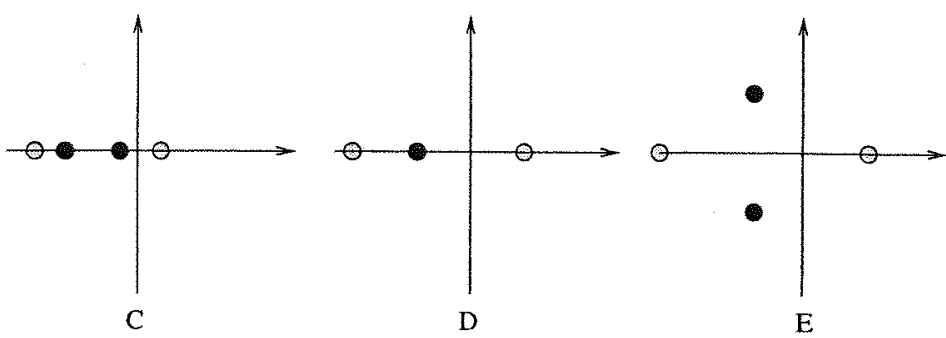

Figura 3.1: $\operatorname{Re} \lambda \times \operatorname{Im} \lambda$

Na figura (3.1) temos:
A) $\phi<\frac{\mu(\alpha+\beta)}{\alpha}$
$(R<1)$
B) $\phi=\frac{\mu(\alpha+\beta)}{\alpha}$
$(R=1)$
C) $\frac{\mu(\alpha+\beta)}{\alpha}<\phi<\frac{1}{4 \alpha}\left[(\alpha+\beta+\mu)^{2}+4 \mu(\alpha+\beta)\right] \quad(R>1)$
D) $\phi=\frac{1}{4 \alpha}\left[(\alpha+\beta+\mu)^{2}+4 \mu(\alpha+\beta)\right]$
E) $\phi>\frac{1}{4 \alpha}\left[(\alpha+\beta+\mu)^{2}+4 \mu(\alpha+\beta)\right]$

\subsection{Os pontos de equilíbrio}

Consideremos, então, o sistema (S), dado por

$$
\left\{\begin{array}{l}
\frac{d P}{d t}=\phi\left(1-\frac{M}{c}\right) M-(\alpha+\beta) P-k P G \\
\frac{d M}{d t}=\alpha P-\mu M \\
\frac{d L}{d t}=\phi^{\prime}\left(1-\frac{G}{c^{\prime}}\right) G-\left(\alpha^{\prime}+\beta^{\prime}\right) L+k^{\prime} P G \\
\frac{d G}{d t}=\alpha^{\prime} L-\mu^{\prime} G
\end{array} .\right.
$$

Lema 3.2.1 Este sistema apresenta quatro pontos de equilibrio dados por:

$$
\left(P_{1}, M_{1}, L_{1}, G_{1}\right)=(0,0,0,0)
$$




$$
\begin{gathered}
\left(P_{2}, M_{2}, L_{2}, G_{2}\right)=\left(\frac{c \mu}{\alpha}\left[1-\frac{1}{R}\right], c\left[1-\frac{1}{R}\right], 0,0\right) \\
\left(P_{3}, M_{3}, L_{3}, G_{3}\right)=\left(0,0, \frac{c^{\prime} \mu^{\prime}}{\alpha^{\prime}}\left[1-\frac{1}{R^{\prime}}\right], c^{\prime}\left[1-\frac{1}{R^{\prime}}\right]\right)
\end{gathered}
$$

$\left(P_{4}, M_{4}, L_{4}, G_{4}\right)$ tal que:

$$
\begin{aligned}
& P_{4}=\frac{c \mu \phi^{\prime}}{\alpha^{2} \phi \phi^{\prime}+\mu^{2} c c^{\prime} k k^{\prime}}\left(\alpha \phi\left[1-\frac{1}{R}\right]-\mu c^{\prime} k\left[1-\frac{1}{R^{\prime}}\right]\right), \\
& M_{4}=\frac{c \alpha \phi^{\prime}}{\alpha^{2} \phi \phi^{\prime}+\mu^{2} c c^{\prime} k k^{\prime}}\left(\alpha \phi\left[1-\frac{1}{R}\right]-\mu c^{\prime} k\left[1-\frac{1}{R^{\prime}}\right]\right), \\
& L_{4}=\frac{c^{\prime} \mu^{\prime} \alpha \phi}{\alpha^{\prime}\left(\alpha^{2} \phi \phi^{\prime}+\mu^{2} c c^{\prime} k k^{\prime}\right)}\left(c \mu k^{\prime}\left[1-\frac{1}{R}\right]+\alpha \phi^{\prime}\left[1-\frac{1}{R^{\prime}}\right]\right), \\
& G_{4}=\frac{c^{\prime} \alpha \phi}{\alpha^{2} \phi \phi^{\prime}+\mu^{2} c c^{\prime} k k^{\prime}}\left(c \mu k^{\prime}\left[1-\frac{1}{R}\right]+\alpha \phi^{\prime}\left[1-\frac{1}{R^{\prime}}\right]\right),
\end{aligned}
$$

onde

$$
R=\frac{\alpha \phi}{\mu(\alpha+\beta)}, \quad R^{\prime}=\frac{\alpha^{\prime} \phi^{\prime}}{\mu^{\prime}\left(\alpha^{\prime}+\beta^{\prime}\right)} \text {. }
$$

Demonstração 3.2.1 Das segunda e quarta equações do sistema

$$
\left\{\begin{aligned}
\phi\left(1-\frac{M}{c}\right) M-(\alpha+\beta) P-k P G & =0 \\
\alpha P-\mu M & =0 \\
\phi^{\prime}\left(1-\frac{G}{c^{\prime}}\right) G-\left(\alpha^{\prime}+\beta^{\prime}\right) L+k^{\prime} P G & =0 \\
\alpha^{\prime} L-\mu^{\prime} G & =0
\end{aligned}\right.
$$

temos

$$
\alpha P-\mu M=0 \Rightarrow P=\frac{\mu}{\alpha} M
$$

$e$

$$
\alpha^{\prime} L-\mu^{\prime} G=0 \Rightarrow L=\frac{\mu^{\prime}}{\alpha^{\prime}} G
$$

Substituindo (3.11) na primeira equação do sistema (3.10) obtemos:

$$
\phi\left(1-\frac{M}{c}\right) M-(\alpha+\beta) \frac{\mu}{\alpha} M-k \frac{\mu}{\alpha} M G=0
$$




$$
\begin{array}{cl}
\Leftrightarrow M\left[\phi\left(1-\frac{M}{c}\right)-\frac{\mu}{\alpha}(\alpha+\beta)-\frac{\mu}{\alpha} k G\right]=0 \\
\Leftrightarrow M=0 \text { ou } \quad \phi\left(1-\frac{M}{c}\right)-\frac{\mu}{\alpha}(\alpha+\beta)-\frac{\mu}{\alpha} k G=0 \\
\Leftrightarrow \frac{\phi}{c} M=\phi-\frac{\mu}{\alpha}(\alpha+\beta)-\frac{\mu}{\alpha} k G \\
\Leftrightarrow M=c\left[1-\frac{1}{R}\right]-\frac{c \mu k}{\alpha \phi} G .
\end{array}
$$

caso $(M=0)$

Da equação (3.11), se $M=0$ temos também que $P=0$. Substituindo (3.11) e (3.12) na terceira equação do sistema (3.10) obtemos:

$$
\begin{aligned}
& \phi^{\prime}\left(1-\frac{G}{c^{\prime}}\right) G-\left(\alpha^{\prime}+\beta^{\prime}\right) \frac{\mu^{\prime}}{\alpha^{\prime}} G+k^{\prime} \frac{\mu}{\alpha} M G=0 \\
& \Leftrightarrow G\left[\phi^{\prime}\left(1-\frac{G}{c^{\prime}}\right)-\frac{\mu^{\prime}}{\alpha^{\prime}}\left(\alpha^{\prime}+\beta^{\prime}\right)+\frac{\mu}{\alpha} k^{\prime} M\right]=0
\end{aligned}
$$

Portanto temos

$G=0$ e, de (3.12), $L=0$, obtendo assim o ponto $\left(P_{1}, M_{1}, L_{1}, G_{1}\right)=(0,0,0,0)$, ou

$$
\begin{gathered}
\phi^{\prime}\left(1-\frac{G}{c^{\prime}}\right)-\frac{\mu^{\prime}}{\alpha^{\prime}}\left(\alpha^{\prime}+\beta^{\prime}\right)=0 \\
\Rightarrow \frac{\phi^{\prime}}{c^{\prime}} G=\phi^{\prime}-\frac{\mu^{\prime}}{\alpha^{\prime}}\left(\alpha^{\prime}+\beta^{\prime}\right) \\
\Rightarrow G=c^{\prime}\left[1-\frac{1}{R^{\prime}}\right]
\end{gathered}
$$

e, portanto, de (3.12),

$$
L=\frac{c^{\prime} \mu^{\prime}}{\alpha^{\prime}}\left[1-\frac{1}{R^{\prime}}\right]
$$

obtendo assim o ponto $\left(P_{3}, M_{3}, L_{3}, G_{3}\right)=\left(0,0, \frac{c^{\prime} \mu^{\prime}}{\alpha^{\prime}}\left[1-\frac{1}{R^{\prime}}\right], c^{\prime}\left[1-\frac{1}{R^{\prime}}\right]\right)$.

caso $\left(M=c\left[1-\frac{1}{R}\right]-\frac{c \mu k}{\alpha \phi} G\right)$

Substituindo (3.11) e (3.12) na terceira equação do sistema (3.10), como no caso anterior, obtemos:

$$
G\left[\phi^{\prime}\left(1-\frac{G}{c^{\prime}}\right)-\frac{\mu^{\prime}}{\alpha^{\prime}}\left(\alpha^{\prime}+\beta^{\prime}\right)+\frac{\mu}{\alpha} k^{\prime} M\right]=0
$$


Portanto temos $G=0 \mathrm{e}$, de (3.12), $L=0 \mathrm{e}$, como $M=c\left[1-\frac{1}{R}\right]-\frac{c \mu k}{\alpha \phi} G$,

$$
M=c\left[1-\frac{1}{R}\right]
$$

$e, \operatorname{de}(3.11)$,

$$
P=\frac{c \mu}{\alpha}\left[1-\frac{1}{R}\right]
$$

obtendo assim o ponto $\left(P_{2}, M_{2}, L_{2}, G_{2}\right)=\left(\frac{c \mu}{\alpha}\left[1-\frac{1}{R}\right], c\left[1-\frac{1}{R}\right], 0,0\right)$, ou temos

$$
\phi^{\prime}\left(1-\frac{G}{c^{\prime}}\right)-\frac{\mu^{\prime}}{\alpha^{\prime}}\left(\alpha^{\prime}+\beta^{\prime}\right)+\frac{\mu}{\alpha} k^{\prime} M=0
$$

que, substituindo $M$ pela sua expressão, obtemos

$$
\begin{gathered}
\phi^{\prime}\left(1-\frac{G}{c^{\prime}}\right)-\frac{\mu^{\prime}}{\alpha^{\prime}}\left(\alpha^{\prime}+\beta^{\prime}\right)+\frac{\mu}{\alpha} k^{\prime}\left(c\left[1-\frac{1}{R}\right]-\frac{c \mu k}{\alpha \phi} G\right)=0 \\
\Leftrightarrow \frac{\phi^{\prime}}{c^{\prime}} G+\frac{c \mu^{2} k k^{\prime}}{\alpha^{2} \phi} G=\phi^{\prime}-\frac{\mu^{\prime}}{\alpha^{\prime}}\left(\alpha^{\prime}+\beta^{\prime}\right)+\frac{\mu}{\alpha} c k^{\prime}\left[1-\frac{1}{R}\right] \\
\Leftrightarrow\left(\frac{\phi^{\prime}}{c^{\prime}}+\frac{c \mu^{2} k k^{\prime}}{\alpha^{2} \phi}\right) G=\phi^{\prime}\left[1-\frac{1}{R^{\prime}}\right]+\frac{\mu}{\alpha} c k^{\prime}\left[1-\frac{1}{R}\right] \\
\Leftrightarrow \quad\left(\frac{\alpha^{2} \phi \phi^{\prime}+\mu^{2} c c^{\prime} k k^{\prime}}{c^{\prime} \alpha^{2} \phi}\right) G=\phi^{\prime}\left[1-\frac{1}{R^{\prime}}\right]+\frac{\mu}{\alpha} c k^{\prime}\left[1-\frac{1}{R}\right] \\
\Leftrightarrow G=\frac{c^{\prime} \alpha^{2} \phi}{\alpha^{2} \phi \phi^{\prime}+\mu^{2} c c^{\prime} k k^{\prime}}\left(\phi^{\prime}\left[1-\frac{1}{R^{\prime}}\right]+\frac{\mu}{\alpha} c k^{\prime}\left[1-\frac{1}{R}\right]\right) \\
\Leftrightarrow G=\frac{c^{\prime} \alpha \phi}{\alpha^{2} \phi \phi^{\prime}+\mu^{2} c c^{\prime} k k^{\prime}}\left(\alpha \phi^{\prime}\left[1-\frac{1}{R^{\prime}}\right]+\mu c k^{\prime}\left[1-\frac{1}{R}\right]\right)
\end{gathered}
$$

$e$, de (3.12),

$$
L=\frac{c^{\prime} \mu^{\prime} \alpha \phi}{\alpha^{\prime}\left(\alpha^{2} \phi \phi^{\prime}+\mu^{2} c c^{\prime} k k^{\prime}\right)}\left(\alpha \phi^{\prime}\left[1-\frac{1}{R^{\prime}}\right]+\mu c k^{\prime}\left[1-\frac{1}{R}\right]\right)
$$

$e$, como $M=c\left[1-\frac{1}{R}\right]-\frac{c \mu k}{\alpha \phi} G$,

$$
M=c\left[1-\frac{1}{R}\right]-\frac{c \mu k}{\alpha \phi} \frac{c^{\prime} \alpha \phi}{\alpha^{2} \phi \phi^{\prime}+\mu^{2} c c^{\prime} k k^{\prime}}\left(\alpha \phi^{\prime}\left[1-\frac{1}{R^{\prime}}\right]+\mu c k^{\prime}\left[1-\frac{1}{R}\right]\right)
$$




$$
\begin{gathered}
\Rightarrow M=c\left[1-\frac{1}{R}\right]-\frac{\mu c c^{\prime} k}{\alpha^{2} \phi \phi^{\prime}+\mu^{2} c c^{\prime} k k^{\prime}}\left(\alpha \phi^{\prime}\left[1-\frac{1}{R^{\prime}}\right]+\mu c k^{\prime}\left[1-\frac{1}{R}\right]\right) \\
\Rightarrow M=\left(\left(\alpha^{2} \phi \phi^{\prime}+\mu^{2} c c^{\prime} k k^{\prime}\right) c\left[1-\frac{1}{R}\right]-\mu c c^{\prime} k \alpha \phi^{\prime}\left[1-\frac{1}{R^{\prime}}\right]-\mu^{2} c^{2} c^{\prime} k k^{\prime}\left[1-\frac{1}{R}\right]\right) \frac{1}{\alpha^{2} \phi \phi^{\prime}+\mu^{2} c c^{\prime} k k^{\prime}} \\
\Rightarrow M=\left(\alpha^{2} \phi \phi^{\prime} c\left[1-\frac{1}{R}\right]+\mu^{2} c^{2} c^{\prime} k k^{\prime}\left[1-\frac{1}{R}\right]-\mu c c^{\prime} k \alpha \phi^{\prime}\left[1-\frac{1}{R^{\prime}}\right]-\mu^{2} c^{2} c^{\prime} k k^{\prime}\left[1-\frac{1}{R}\right]\right) \frac{1}{\alpha^{2} \phi \phi^{\prime}+\mu^{2} c c^{\prime} k k^{\prime}} \\
\Rightarrow M=\left(\alpha^{2} \phi \phi^{\prime} c\left[1-\frac{1}{R}\right]-\mu c c^{\prime} k \alpha \phi^{\prime}\left[1-\frac{1}{R^{\prime}}\right]\right) \frac{1}{\alpha^{2} \phi \phi^{\prime}+\mu^{2} c c^{\prime} k k^{\prime}} \\
\Rightarrow M=\frac{c \alpha \phi^{\prime}}{\alpha^{2} \phi \phi^{\prime}+\mu^{2} c c^{\prime} k k^{\prime}}\left(\alpha \phi\left[1-\frac{1}{R}\right]-\mu c^{\prime} k\left[1-\frac{1}{R^{\prime}}\right]\right)
\end{gathered}
$$

e, de (3.11)

$$
P=\frac{c \mu \phi^{\prime}}{\alpha^{2} \phi \phi^{\prime}+\mu^{2} c c^{\prime} k k^{\prime}}\left(\alpha \phi\left[1-\frac{1}{R}\right]-\mu c^{\prime} k\left[1-\frac{1}{R^{\prime}}\right]\right)
$$

obtendo assim o ponto $\left(P_{4}, M_{4}, L_{4}, G_{4}\right)$.

Observação 3.2.1 Note que $R>0$ e $R^{\prime}>0$, uma vez que todos os parâmetros são estritamente positivos (observação 1.2.1).

Observação 3.2.2 Para que o ponto $\left(P_{2}, M_{2}, L_{2}, G_{2}\right)$, dado por (3.7), exista no sentido biológico, é necessário e suficiente que $R \geq 1$, caso contrário as populações ficariam determinadas por um número negativo de individuos. Do mesmo modo, para o ponto $\left(P_{3}, M_{3}, L_{3}, G_{3}\right)$, dado por (3.8), necessitamos $R^{\prime} \geq 1$.

Observação 3.2.3 Para que tenhamos $P_{4}>0, M_{4}>0, L_{4}>0, G_{4}>0$ em (3.9), das expressões de $L_{4}$ e $G_{4}$, note que é suficiente $R>1$ e $R^{\prime}>1$. Quanto aos valores de $P_{4} e$ $M_{4}$ discutiremos no seguinte lema:

Lema 3.2.2 Sejam $k_{\text {max }}=\frac{\alpha \phi\left[1-\frac{1}{R}\right]}{c^{\prime} \mu\left[1-\frac{1}{R^{\prime}}\right]}$ e $k_{\text {min }}^{\prime}=-\frac{\alpha \phi^{\prime}\left[1-\frac{1}{R^{\prime}}\right]}{c \mu\left[1-\frac{1}{R}\right]}$. Então para que $P_{4}>0, M_{4}>0$, $L_{4}>0$ e $G_{4}>0$, devemos ter:
(a) $R>1, R^{\prime}>1$ ek< $k_{\max }$, ou
(b) $R>1$ e $R^{\prime}=1$, ou
(c) $R>1, R^{\prime}<1 e k^{\prime}>k_{\min }^{\prime}$. 
Demonstração 3.2.2 Notemos inicialmente que $R>1$ é condição necessária para que $P_{4}>0, M_{4}>0, L_{4}>0$ e $G_{4}>0$, como pede o lema.

De fato, se $R \leq 1$, temos

$$
\alpha \phi\left[1-\frac{1}{R}\right] \leq 0
$$

o que implica, das expressões de $P_{4}$ e $M_{4}$, dadas em (3.9), que deveríamos ter

$$
\begin{gathered}
-\mu c^{\prime} k\left[1-\frac{1}{R^{\prime}}\right]>0, \\
\Rightarrow\left[1-\frac{1}{R^{\prime}}\right]<0 \\
\Rightarrow R^{\prime}<1 .
\end{gathered}
$$

Por outro lado, se $R \leq 1$, temos

$$
c \mu k^{\prime}\left[1-\frac{1}{R}\right] \leq 0
$$

o que implica, das expressões de $L_{4}$ e $G_{4}$, dadas em (3.9), que deveríamos ter

$$
\begin{gathered}
\alpha \phi^{\prime}\left[1-\frac{1}{R^{\prime}}\right]>0, \\
\Rightarrow\left[1-\frac{1}{R^{\prime}}\right]>0, \\
\Rightarrow R^{\prime}>1 .
\end{gathered}
$$

Sendo assim, suponha a partir de agora que $R>1$.

\section{caso (a)}

Se $R^{\prime}>1, L_{4}$ e $G_{4}$, dadas em (3.9), são claramente positivas. Para que tenhamos também $P_{4}>0$ e $M_{4}>0$, basta que

$$
\begin{gathered}
\alpha \phi\left[1-\frac{1}{R}\right]-\mu c^{\prime} k\left[1-\frac{1}{R^{\prime}}\right]>0, \\
\Leftrightarrow \mu c^{\prime} k\left[1-\frac{1}{R^{\prime}}\right]<\alpha \phi\left[1-\frac{1}{R}\right], \\
\Leftrightarrow k<\frac{\alpha \phi\left[1-\frac{1}{R}\right]}{c^{\prime} \mu\left[1-\frac{1}{R^{\prime}}\right]} .
\end{gathered}
$$


Note que neste caso, como $R>1$ e $R^{\prime}>1$, temos $k_{\max }=\frac{\alpha \phi\left[1-\frac{1}{R}\right]}{c^{\prime} \mu\left[1-\frac{1}{R^{\prime}}\right]}$ positivo.

caso (b)

Se $R^{\prime}=1$, os termos que poderiam ser negativos nas expressões de $P_{4}$ e $M_{4}$ desaparecem.

caso (c)

Se $R^{\prime}<1, P_{4}$ e $M_{4}$, dadas em (3.9), são claramente positivas. Para que tenhamos também $L_{4}>0$ e $G_{4}>0$, basta que

$$
\begin{gathered}
c \mu k^{\prime}\left[1-\frac{1}{R}\right]+\alpha \phi^{\prime}\left[1-\frac{1}{R^{\prime}}\right]>0, \\
\Leftrightarrow c \mu k^{\prime}\left[1-\frac{1}{R}\right]>-\alpha \phi^{\prime}\left[1-\frac{1}{R^{\prime}}\right], \\
\Leftrightarrow k^{\prime}>-\frac{\alpha \phi^{\prime}\left[1-\frac{1}{R^{\prime}}\right]}{c \mu\left[1-\frac{1}{R}\right]} .
\end{gathered}
$$

Note que neste caso, como $R>1$ e $R^{\prime}<1$, temos $k_{\min }^{\prime}=-\frac{\alpha \phi^{\prime}\left[1-\frac{1}{R^{\prime}}\right]}{c \mu\left[1-\frac{1}{R}\right]}$ positivo.

\subsection{Análise do espectro}

Seja $J(x), x=(P, M, L, G) \in \mathbb{R}^{4}$, a matriz Jacobiana de (S), dada por

$$
J(x)=\left(\begin{array}{cccc}
-\alpha-\beta-k G & \phi-\frac{2 \phi M}{c} & 0 & -k P \\
\alpha & -\mu & 0 & 0 \\
k^{\prime} G & 0 & -\alpha^{\prime}-\beta^{\prime} & \phi^{\prime}-\frac{2 \phi^{\prime} G}{c^{\prime}}+k^{\prime} P \\
0 & 0 & \alpha^{\prime} & -\mu^{\prime}
\end{array}\right)
$$

\subsection{1 $\left(\mathrm{P}_{1}, \mathrm{M}_{1}, \mathrm{~L}_{1}, \mathrm{G}_{1}\right)$}

Lema 3.3.1 O sistema $(\mathrm{S})$ no ponto $\left(P_{1}, M_{1}, L_{1}, G_{1}\right)=(0,0,0,0)$ apresenta quatro autovalores dados por: 


$$
\begin{aligned}
& \lambda_{1}=-\frac{1}{2}(\alpha+\beta+\mu)+\frac{1}{2} \sqrt{(\alpha+\beta+\mu)^{2}+4 \alpha \phi\left[1-\frac{1}{R}\right]}, \\
& \lambda_{2}=-\frac{1}{2}(\alpha+\beta+\mu)-\frac{1}{2} \sqrt{(\alpha+\beta+\mu)^{2}+4 \alpha \phi\left[1-\frac{1}{R}\right]}, \\
& \lambda_{3}=-\frac{1}{2}\left(\alpha^{\prime}+\beta^{\prime}+\mu^{\prime}\right)+\frac{1}{2} \sqrt{\left(\alpha^{\prime}+\beta^{\prime}+\mu^{\prime}\right)^{2}+4 \alpha^{\prime} \phi^{\prime}\left[1-\frac{1}{R^{\prime}}\right]} \\
& \lambda_{4}=-\frac{1}{2}\left(\alpha^{\prime}+\beta^{\prime}+\mu^{\prime}\right)-\frac{1}{2} \sqrt{\left(\alpha^{\prime}+\beta^{\prime}+\mu^{\prime}\right)^{2}+4 \alpha^{\prime} \phi^{\prime}\left[1-\frac{1}{R^{\prime}}\right]} .
\end{aligned}
$$

Demonstração 3.3.1 Calculando $J(x)$ para $x=(0,0,0,0)$, obtemos

$$
J(0,0,0,0)=\left(\begin{array}{cccc}
-\alpha-\beta & \phi & 0 & 0 \\
\alpha & -\mu & 0 & 0 \\
0 & 0 & -\alpha^{\prime}-\beta^{\prime} & \phi^{\prime} \\
0 & 0 & \alpha^{\prime} & -\mu^{\prime}
\end{array}\right)
$$

Basta agora resolver a equação

$$
\operatorname{det}(J(0,0,0,0)-\lambda I)=0
$$

onde $I$ representa a matriz identidade, obtendo a equação:

$$
\begin{gathered}
{[(\alpha+\beta+\lambda)(\mu+\lambda)-\alpha \phi]\left[\left(\alpha^{\prime}+\beta^{\prime}+\lambda\right)\left(\mu^{\prime}+\lambda\right)-\alpha^{\prime} \phi^{\prime}\right]=0} \\
{\left[\lambda^{2}+(\alpha+\beta+\mu) \lambda+\mu(\alpha+\beta)-\alpha \phi\right]\left[\lambda^{2}+\left(\alpha^{\prime}+\beta^{\prime}+\mu^{\prime}\right) \lambda+\mu^{\prime}\left(\alpha^{\prime}+\beta^{\prime}\right)-\alpha^{\prime} \phi^{\prime}\right]=0 .}
\end{gathered}
$$

\section{Portanto,}

$$
\begin{aligned}
\lambda & =-\frac{1}{2}(\alpha+\beta+\mu) \pm \frac{1}{2} \sqrt{(\alpha+\beta+\mu)^{2}-4[\mu(\alpha+\beta)-\alpha \phi]} \\
& =-\frac{1}{2}(\alpha+\beta+\mu) \pm \frac{1}{2} \sqrt{(\alpha+\beta+\mu)^{2}+4 \alpha \phi\left[1-\frac{1}{R}\right]}
\end{aligned}
$$

ou,

$$
\begin{aligned}
\lambda & =-\frac{1}{2}\left(\alpha^{\prime}+\beta^{\prime}+\mu^{\prime}\right) \pm \frac{1}{2} \sqrt{\left(\alpha^{\prime}+\beta^{\prime}+\mu^{\prime}\right)^{2}-4\left[\mu^{\prime}\left(\alpha^{\prime}+\beta^{\prime}\right)-\alpha^{\prime} \phi^{\prime}\right]} \\
& =-\frac{1}{2}\left(\alpha^{\prime}+\beta^{\prime}+\mu^{\prime}\right) \pm \frac{1}{2} \sqrt{\left(\alpha^{\prime}+\beta^{\prime}+\mu^{\prime}\right)^{2}+4 \alpha^{\prime} \phi^{\prime}\left[1-\frac{1}{R^{\prime}}\right]}
\end{aligned}
$$


Lema 3.3.2 Os autovalores $\lambda_{1}, \lambda_{2}, \lambda_{3}$ e $\lambda_{4}$, definidos em (3.14), são reais.

Demonstração 3.3.2 Se chamarmos de $\Delta e \Delta^{\prime}$ as expressões que aparecem dentro dos radicais de, respectivamente, $\lambda_{1}, \lambda_{2}$ e $\lambda_{3}, \lambda_{4}$ em (3.14), tendo assim,

$$
\Delta=(\alpha+\beta+\mu)^{2}+4 \alpha \phi\left[1-\frac{1}{R}\right] e \Delta^{\prime}=\left(\alpha^{\prime}+\beta^{\prime}+\mu^{\prime}\right)^{2}+4 \alpha^{\prime} \phi^{\prime}\left[1-\frac{1}{R^{\prime}}\right],
$$

para provarmos o lema basta mostrar que $\Delta>0$ e $\Delta^{\prime}>0$ para quaisquer valores de $R$ e $R^{\prime}$. De fato,

$$
\begin{aligned}
\Delta & =(\alpha+\beta+\mu)^{2}+4 \alpha \phi\left[1-\frac{1}{R}\right] \\
& =(\alpha+\beta+\mu)^{2}+4 \alpha \phi-4 \mu(\alpha+\beta) \\
& =(\alpha+\beta-\mu)^{2}+4 \alpha \phi .
\end{aligned}
$$

Analogamente,

$$
\begin{aligned}
\Delta^{\prime} & =\left(\alpha^{\prime}+\beta^{\prime}+\mu^{\prime}\right)^{2}+4 \alpha^{\prime} \phi^{\prime}\left[1-\frac{1}{R^{\prime}}\right] \\
& =\left(\alpha^{\prime}+\beta^{\prime}+\mu^{\prime}\right)^{2}+4 \alpha^{\prime} \phi^{\prime}-4 \mu^{\prime}\left(\alpha^{\prime}+\beta^{\prime}\right) \\
& =\left(\alpha^{\prime}+\beta^{\prime}-\mu^{\prime}\right)^{2}+4 \alpha^{\prime} \phi^{\prime} .
\end{aligned}
$$

Lema 3.3.3 $O$ ponto $\left(P_{1}, M_{1}, L_{1}, G_{1}\right)=(0,0,0,0)$ é:

(a) uma sela 2-2 se $R>1$ e $R^{\prime}>1$,

(b) uma sela 3-1 se $R>1$ e $R^{\prime}<1$ ou se $R<1$ e $R^{\prime}>1$,

(c) um nó atrator se $R<1$ e $R^{\prime}<1$.

Demonstração 3.3.3 Pelo lema (3.3.2) fica claro que os autovalores $\lambda_{2}$ e $\lambda_{4}$, dados por (3.14), são negativos. Analisemos, então, os outros dois autovalores:

Se $R>1$ a expressão

$$
4 \alpha \phi\left[1-\frac{1}{R}\right]
$$

é positiva, o que implica por (3.14), $\lambda_{1}>0$. 
Se $R<1$ a expressão

$$
4 \alpha \phi\left[1-\frac{1}{R}\right]
$$

é negativa, mas a expressão

$$
(\alpha+\beta+\mu)^{2}+4 \alpha \phi\left[1-\frac{1}{R}\right]
$$

é positiva (lema 3.3.2), o que implica por (3.14), $\lambda_{1}<0$.

O mesmo argumento vale se $R^{\prime}>1$, implicando $\lambda_{3}>0$; e, se $R^{\prime}<1$, implicando $\lambda_{3}<0$. Portanto,

(a) se $R>1$ e $R^{\prime}>1 \Rightarrow \lambda_{1}>0$ e $\lambda_{3}>0$,

(b) se $R>1$ e $R^{\prime}<1 \Rightarrow \lambda_{1}>0$ e $\lambda_{3}<0$, ou se $R<1$ e $R^{\prime}>1 \Rightarrow \lambda_{1}<0$ e $\lambda_{3}>0$,

(c) se $R<1$ e $R^{\prime}<1 \Rightarrow \lambda_{1}<0$ e $\lambda_{3}<0$.

\subsection{2 $\left(\mathrm{P}_{2}, \mathrm{M}_{2}, \mathrm{~L}_{2}, \mathrm{G}_{2}\right)$}

Lema 3.3.4 $O$ sistema (S) no ponto $\left(P_{2}, M_{2}, L_{2}, G_{2}\right)$, dado por (3.7), apresenta quatro autovalores dados por:

$$
\begin{aligned}
& \lambda_{1}=-\frac{1}{2}(\alpha+\beta+\mu)+\frac{1}{2} \sqrt{(\alpha+\beta+\mu)^{2}-4 \alpha \phi\left[1-\frac{1}{R}\right]}, \\
& \lambda_{2}=-\frac{1}{2}(\alpha+\beta+\mu)-\frac{1}{2} \sqrt{(\alpha+\beta+\mu)^{2}-4 \alpha \phi\left[1-\frac{1}{R}\right]}, \\
& \lambda_{3}=-\frac{1}{2}\left(\alpha^{\prime}+\beta^{\prime}+\mu^{\prime}\right)+\frac{1}{2} \sqrt{\left(\alpha^{\prime}+\beta^{\prime}+\mu^{\prime}\right)^{2}+4 \alpha^{\prime} \phi^{\prime}\left[1-\frac{1}{R^{\prime}}\right]+4 \frac{c \alpha^{\prime} \mu}{\alpha}\left[1-\frac{1}{R}\right] k^{\prime}}, \\
& \lambda_{4}=-\frac{1}{2}\left(\alpha^{\prime}+\beta^{\prime}+\mu^{\prime}\right)-\frac{1}{2} \sqrt{\left(\alpha^{\prime}+\beta^{\prime}+\mu^{\prime}\right)^{2}+4 \alpha^{\prime} \phi^{\prime}\left[1-\frac{1}{R^{\prime}}\right]+4 \frac{c \alpha^{\prime} \mu}{\alpha}\left[1-\frac{1}{R}\right] k^{\prime}} .
\end{aligned}
$$

Demonstração 3.3.4 Calculando $J(x)$ para $x=\left(P_{2}, M_{2}, L_{2}, G_{2}\right)$, dado por (3.7), obtemos

$$
J\left(P_{2}, M_{2}, L_{2}, G_{2}\right)=\left(\begin{array}{cccc}
-\alpha-\beta & \phi-2 \phi\left[1-\frac{1}{R}\right] & 0 & -\frac{c \mu}{\alpha}\left[1-\frac{1}{R}\right] k \\
\alpha & -\mu & 0 & 0 \\
0 & 0 & -\alpha^{\prime}-\beta^{\prime} & \phi^{\prime}+\frac{c \mu}{\alpha}\left[1-\frac{1}{R}\right] k^{\prime} \\
0 & 0 & \alpha^{\prime} & -\mu^{\prime}
\end{array}\right)
$$


Basta agora resolver a equação

$$
\operatorname{det}\left(J\left(P_{2}, M_{2}, L_{2}, G_{2}\right)-\lambda I\right)=0
$$

onde I representa a matriz identidade, obtendo, assim, a equação:

$\left[(\mu+\lambda)(\alpha+\beta+\lambda)-\alpha\left(\phi-2 \phi\left[1-\frac{1}{R}\right]\right)\right]\left[\left(\mu^{\prime}+\lambda\right)\left(\alpha^{\prime}+\beta^{\prime}+\lambda\right)-\alpha^{\prime}\left(\phi^{\prime}+\frac{c \mu}{\alpha}\left[1-\frac{1}{R}\right] k^{\prime}\right)\right]=0$.

Temos, então, que:

$$
\begin{aligned}
& \left.\lambda^{2}+(\alpha+\beta+\mu) \lambda+\mu(\alpha+\beta)-\alpha \phi+2 \alpha \phi\left[1-\frac{1}{R}\right]\right)=0 \\
& \left.\lambda^{2}+(\alpha+\beta+\mu) \lambda+\mu(\alpha+\beta)-\alpha \phi+2 \alpha \phi-\frac{2 \alpha \phi}{R}\right)=0 \\
& \lambda^{2}+(\alpha+\beta+\mu) \lambda+\alpha \phi-\mu(\alpha+\beta)=0
\end{aligned}
$$

$o u$,

$$
\lambda^{2}+\left(\alpha^{\prime}+\beta^{\prime}+\mu^{\prime}\right) \lambda+\mu^{\prime}\left(\alpha^{\prime}+\beta^{\prime}\right)-\alpha^{\prime} \phi^{\prime}-\frac{c \alpha^{\prime} \mu}{\alpha}\left[1-\frac{1}{R}\right] k^{\prime}=0
$$

Portanto,

$$
\begin{aligned}
\lambda & =-\frac{1}{2}(\alpha+\beta+\mu) \pm \frac{1}{2} \sqrt{(\alpha+\beta+\mu)^{2}-4[\alpha \phi-\mu(\alpha+\beta)]} \\
& =-\frac{1}{2}(\alpha+\beta+\mu) \pm \frac{1}{2} \sqrt{(\alpha+\beta+\mu)^{2}-4 \alpha \phi\left[1-\frac{1}{R}\right]}
\end{aligned}
$$

ou,

$$
\begin{aligned}
\lambda & =-\frac{1}{2}\left(\alpha^{\prime}+\beta^{\prime}+\mu^{\prime}\right) \pm \frac{1}{2} \sqrt{\left(\alpha^{\prime}+\beta^{\prime}+\mu^{\prime}\right)^{2}-4\left[\mu^{\prime}\left(\alpha^{\prime}+\beta^{\prime}\right)-\alpha^{\prime} \phi^{\prime}-\frac{c \alpha^{\prime} \mu}{\alpha}\left[1-\frac{1}{R}\right] k^{\prime}\right]} \\
& =-\frac{1}{2}\left(\alpha^{\prime}+\beta^{\prime}+\mu^{\prime}\right) \pm \frac{1}{2} \sqrt{\left(\alpha^{\prime}+\beta^{\prime}+\mu^{\prime}\right)^{2}+4 \alpha^{\prime} \phi^{\prime}\left[1-\frac{1}{R^{\prime}}\right]+4 \frac{c \alpha^{\prime} \mu}{\alpha}\left[1-\frac{1}{R}\right] k^{\prime}} .
\end{aligned}
$$

Lema 3.3.5 Se $R>1$ os autovalores $\lambda_{3}$ e $\lambda_{4}$, dados por (3.15), são reais.

Demonstração 3.3.5 A expressão

$$
\left(\alpha^{\prime}+\beta^{\prime}+\mu^{\prime}\right)^{2}+4 \alpha^{\prime} \phi^{\prime}\left[1-\frac{1}{R^{\prime}}\right]+4 \frac{c \alpha^{\prime} \mu}{\alpha}\left[1-\frac{1}{R}\right] k^{\prime}
$$


que aparece dentro dos radicais de $\lambda_{3}$ e $\lambda_{4}$, em (3.15), tem seu terceiro termo

$$
4 \frac{c \alpha^{\prime} \mu}{\alpha}\left[1-\frac{1}{R}\right] k^{\prime}
$$

positivo, se $R>1$ como pede a hipótese. Basta, então, mostrarmos que os dois primeiros termos são sempre positivos.

De fato,

$$
\begin{aligned}
& \left(\alpha^{\prime}+\beta^{\prime}+\mu^{\prime}\right)^{2}+4 \alpha^{\prime} \phi^{\prime}\left[1-\frac{1}{R^{\prime}}\right]= \\
& =\left(\alpha^{\prime}+\beta^{\prime}+\mu^{\prime}\right)^{2}+4 \alpha^{\prime} \phi^{\prime}-4 \mu^{\prime}\left(\alpha^{\prime}+\beta^{\prime}\right) \\
& =\left(\alpha^{\prime}+\beta^{\prime}-\mu^{\prime}\right)^{2}+4 \alpha^{\prime} \phi^{\prime} .
\end{aligned}
$$

Seja $k_{\min }^{\prime}=-\frac{\alpha \phi^{\prime}\left[1-\frac{1}{R^{\prime}}\right]}{c \mu\left[1-\frac{1}{R}\right]}$ como definido no lema 3.2.2.

Lema 3.3.6 $O$ ponto $\left(P_{2}, M_{2}, L_{2}, G_{2}\right)$, dado por (3.7) tem indice de estabilidade:

$$
\begin{aligned}
& \left(\mathbf{a}_{1}\right) \sigma=\mathbf{3} \text { se } R>1 \text { e } R^{\prime} \geq 1, \\
& \left(\mathbf{a}_{2}\right) \sigma=\mathbf{3} \text { se } R>1, R^{\prime}<1 \text { e } k^{\prime}>k_{\text {min }}^{\prime}, \\
& \left(\mathbf{a}_{3}\right) \sigma=4 \text { se } R>1, R^{\prime}<1 \text { e } k^{\prime}<k_{\text {min }}^{\prime}, \\
& \left(\mathbf{b}_{1}\right) \sigma=\mathbf{2} \text { se } R<1, \text { e } k^{\prime}<k_{\text {min }}^{\prime}, \\
& \left(\mathbf{b}_{2}\right) \sigma=3 \text { se } R<1, \text { e } k^{\prime}>k_{\text {min }}^{\prime} .
\end{aligned}
$$

\section{Demonstração 3.3.6}

(a) Suponha $R>1$.

Então, $\lambda_{4}<0$ (lema 3.3.5), e o termo

$$
-4 \alpha \phi\left[1-\frac{1}{R}\right]
$$

que aparece dentro do radical nas expressões de $\lambda_{1}$ e $\lambda_{2}$, dadas em (3.15), é negativo, implicando que $\lambda_{1}$ e $\lambda_{2}$ são complexos, não reais, com parte real negativa, ou são ambos reais $e$ negativos.

(a) $R^{\prime} \geq 1 \Rightarrow \lambda_{3}>0$.

Basta ver que o segundo e o terceiro termo dentro do radical de $\lambda_{3}$ em (3.15) são positivos. 
( $\left.\mathrm{a}_{2}\right) R^{\prime}<1$ e $k^{\prime}>k_{\min }^{\prime} \Rightarrow \lambda_{3}>0$.

Basta ver que a soma do segundo com o terceiro termo dentro do radical de $\lambda_{3}$ em (3.15) é positiva, pois

$$
\begin{gathered}
4 \alpha^{\prime} \phi^{\prime}\left[1-\frac{1}{R^{\prime}}\right]+4 \frac{c \alpha^{\prime} \mu}{\alpha}\left[1-\frac{1}{R}\right] k^{\prime}>0 \\
\Leftrightarrow 4 \frac{c \alpha^{\prime} \mu}{\alpha}\left[1-\frac{1}{R}\right] k^{\prime}>-4 \alpha^{\prime} \phi^{\prime}\left[1-\frac{1}{R^{\prime}}\right] \\
\Leftrightarrow k^{\prime}>-\frac{\alpha \phi^{\prime}\left[1-\frac{1}{R^{\prime}}\right]}{c \mu\left[1-\frac{1}{R}\right]} .
\end{gathered}
$$

(a) $R^{\prime}<1 e k^{\prime}<k_{\min }^{\prime} \Rightarrow \lambda_{3}<0$.

Basta ver que a soma do segundo com o terceiro termo dentro do radical de $\lambda_{3} \mathrm{em}$ (3.15) é negativa, mas a soma dos três termos é positiva (lema 3.3.5).

(b) Suponha $R<1$.

Então $\lambda_{1}>0$ e $\lambda_{2}<0$ pois $-4 \alpha \phi\left[1-\frac{1}{R}\right]>0$.

(b $\mathrm{b}_{1}$ Se $k^{\prime}<k_{\min }^{\prime} \Rightarrow \lambda_{3}>0$ e $\lambda_{4}<0$.

Basta ver que a soma do segundo com o terceiro termo dentro do radical de $\lambda_{3}$ e $\lambda_{4}$ em (3.15) é positiva, pois

$$
\begin{gathered}
4 \alpha^{\prime} \phi^{\prime}\left[1-\frac{1}{R^{\prime}}\right]+4 \frac{c \alpha^{\prime} \mu}{\alpha}\left[1-\frac{1}{R}\right] k^{\prime}>0 \\
\Leftrightarrow 4 \frac{c \alpha^{\prime} \mu}{\alpha}\left[1-\frac{1}{R}\right] k^{\prime}>-4 \alpha^{\prime} \phi^{\prime}\left[1-\frac{1}{R^{\prime}}\right] \\
\Leftrightarrow k^{\prime}<-\frac{\alpha \phi^{\prime}\left[1-\frac{1}{R^{\prime}}\right]}{c \mu\left[1-\frac{1}{R}\right]} .
\end{gathered}
$$

$\left(\mathrm{b}_{2}\right)$ Se $k^{\prime}>k_{\text {min }}^{\prime}$, então $4 \alpha^{\prime} \phi^{\prime}\left[1-\frac{1}{R^{\prime}}\right]+4 \frac{c \alpha^{\prime} \mu}{\alpha}\left[1-\frac{1}{R}\right] k^{\prime}<0$. Portanto $\lambda_{3}$ e $\lambda_{4}$ são complexos, não reais, com parte real negativa, ou são ambos reais e negativos.

Lema 3.3.7 $\lambda_{1}$ e $\lambda_{2}$, dados por (3.15), são:

(a) complexos conjugados, não reais, se $\phi>\frac{1}{4 \alpha}\left[(\alpha+\beta+\mu)^{2}+4 \mu(\alpha+\beta)\right]$, ou

(b) reais, se $\phi \leq \frac{1}{4 \alpha}\left[(\alpha+\beta+\mu)^{2}+4 \mu(\alpha+\beta)\right]$. 
Demonstração 3.3.7 Seja

$$
\begin{aligned}
\Delta & =(\alpha+\beta+\mu)^{2}-4 \alpha \phi\left[1-\frac{1}{R}\right] \\
& =(\alpha+\beta+\mu)^{2}+4 \mu(\alpha+\beta)-4 \alpha \phi
\end{aligned}
$$

a expressão que aparece dentro do radical de $\lambda_{1}$ e $\lambda_{2}$ em (3.15).

Temos que:

(a) se $\phi>\frac{1}{4 \alpha}\left[(\alpha+\beta+\mu)^{2}+4 \mu(\alpha+\beta)\right] \Rightarrow \Delta<0 \Rightarrow$ por (3.15), $\lambda_{1}$ e $\lambda_{2}$ são complexos conjugados, não reais $e$,

(b) se $\phi \leq \frac{1}{4 \alpha}\left[(\alpha+\beta+\mu)^{2}+4 \mu(\alpha+\beta)\right] \Rightarrow \Delta \geq 0 \Rightarrow \operatorname{por}(3.15), \lambda_{1}$ e $\lambda_{2}$ são reais.

\subsection{3 $\left(\mathrm{P}_{3}, \mathrm{M}_{3}, \mathrm{~L}_{3}, \mathrm{G}_{3}\right)$}

Lema 3.3.8 $O$ sistema $(\mathrm{S})$ no ponto $\left(P_{3}, M_{3}, L_{3}, G_{3}\right)$, dado por (3.8), apresenta quatro autovalores dados por:

$$
\begin{aligned}
& \lambda_{1}=-\frac{1}{2}\left(\alpha+\beta+\mu+c^{\prime} k\left[1-\frac{1}{R^{\prime}}\right]\right)+\frac{1}{2} \sqrt{\left(\alpha+\beta-\mu+c^{\prime} k\left[1-\frac{1}{R^{\prime}}\right]\right)^{2}+4 \alpha \phi}, \\
& \lambda_{2}=-\frac{1}{2}\left(\alpha+\beta+\mu+c^{\prime} k\left[1-\frac{1}{R^{\prime}}\right]\right)-\frac{1}{2} \sqrt{\left(\alpha+\beta-\mu+c^{\prime} k\left[1-\frac{1}{R^{\prime}}\right]\right)^{2}+4 \alpha \phi}, \\
& \lambda_{3}=-\frac{1}{2}\left(\alpha^{\prime}+\beta^{\prime}+\mu^{\prime}\right)+\frac{1}{2} \sqrt{\left(\alpha^{\prime}+\beta^{\prime}+\mu^{\prime}\right)^{2}-4 \alpha^{\prime} \phi^{\prime}\left[1-\frac{1}{R^{\prime}}\right]}, \\
& \lambda_{4}=-\frac{1}{2}\left(\alpha^{\prime}+\beta^{\prime}+\mu^{\prime}\right)-\frac{1}{2} \sqrt{\left(\alpha^{\prime}+\beta^{\prime}+\mu^{\prime}\right)^{2}-4 \alpha^{\prime} \phi^{\prime}\left[1-\frac{1}{R^{\prime}}\right]} .
\end{aligned}
$$

Demonstração 3.3.8 Calculando $J(x)$ para $x=\left(P_{3}, M_{3}, L_{3}, G_{3}\right)$ obtemos:

$$
J\left(P_{3}, M_{3}, L_{3}, G_{3}\right)=\left(\begin{array}{cccc}
-\alpha-\beta-c^{\prime}\left[1-\frac{1}{R^{\prime}}\right] k & \phi & 0 & 0 \\
\alpha & -\mu & 0 & 0 \\
c^{\prime}\left[1-\frac{1}{R^{\prime}}\right] k^{\prime} & 0 & -\alpha^{\prime}-\beta^{\prime} & \phi^{\prime}-2 \phi^{\prime}\left[1-\frac{1}{R^{\prime}}\right] \\
0 & 0 & \alpha^{\prime} & -\mu^{\prime}
\end{array}\right)
$$

Basta agora resolver a equação

$$
\operatorname{det}\left(J\left(P_{3}, M_{3}, L_{3}, G_{3}\right)-\lambda I\right)=0
$$

onde I representa a matriz identidade, obtendo, assim:

$$
\left[(\mu+\lambda)\left(\alpha+\beta+c^{\prime}\left[1-\frac{1}{R^{\prime}}\right] k+\lambda\right)-\alpha \phi\right]\left[\left(\mu^{\prime}+\lambda\right)\left(\alpha^{\prime}+\beta^{\prime}+\lambda\right)-\alpha^{\prime}\left(\phi^{\prime}-2 \phi^{\prime}\left[1-\frac{1}{R^{\prime}}\right]\right)\right]=0
$$


Temos, então, que:

$$
\lambda^{2}+\left(\alpha+\beta+\mu+c^{\prime}\left[1-\frac{1}{R^{\prime}}\right] k\right) \lambda+\mu\left(\alpha+\beta+c^{\prime}\left[1-\frac{1}{R^{\prime}}\right] k\right)-\alpha \phi=0
$$

ou,

$$
\begin{aligned}
& \lambda^{2}+\left(\alpha^{\prime}+\beta^{\prime}+\mu^{\prime}\right) \lambda+\mu^{\prime}\left(\alpha^{\prime}+\beta^{\prime}\right)-\alpha^{\prime} \phi^{\prime}+2 \alpha^{\prime} \phi^{\prime}\left[1-\frac{1}{R^{\prime}}\right]=0 \\
& \lambda^{2}+\left(\alpha^{\prime}+\beta^{\prime}+\mu^{\prime}\right) \lambda-\mu^{\prime}\left(\alpha^{\prime}+\beta^{\prime}\right)+\alpha^{\prime} \phi^{\prime}=0 .
\end{aligned}
$$

Portanto,

$$
\begin{aligned}
\lambda= & -\frac{1}{2}\left(\alpha+\beta+\mu+c^{\prime}\left[1-\frac{1}{R^{\prime}}\right] k\right) \\
& \pm \frac{1}{2} \sqrt{\left(\alpha+\beta+\mu+c^{\prime}\left[1-\frac{1}{R^{\prime}}\right] k\right)^{2}-4\left[\mu\left(\alpha+\beta+c^{\prime}\left[1-\frac{1}{R^{\prime}}\right] k\right)-\alpha \phi\right]} \\
= & -\frac{1}{2}\left(\alpha+\beta+\mu+c^{\prime}\left[1-\frac{1}{R^{\prime}}\right] k\right) \pm \frac{1}{2} \sqrt{\left(\alpha+\beta-\mu+c^{\prime}\left[1-\frac{1}{R^{\prime}}\right] k\right)^{2}+4 \alpha \phi}
\end{aligned}
$$

$o u$,

$$
\begin{aligned}
\lambda & =-\frac{1}{2}\left(\alpha^{\prime}+\beta^{\prime}+\mu^{\prime}\right) \pm \frac{1}{2} \sqrt{\left(\alpha^{\prime}+\beta^{\prime}+\mu^{\prime}\right)^{2}-4\left[-\mu^{\prime}\left(\alpha^{\prime}+\beta^{\prime}\right)+\alpha^{\prime} \phi^{\prime}\right]} \\
& =-\frac{1}{2}\left(\alpha^{\prime}+\beta^{\prime}+\mu^{\prime}\right) \pm \frac{1}{2} \sqrt{\left(\alpha^{\prime}+\beta^{\prime}+\mu^{\prime}\right)^{2}-4 \alpha^{\prime} \phi^{\prime}\left[1-\frac{1}{R^{\prime}}\right]}
\end{aligned}
$$

Seja $k_{\text {max }}=\frac{\alpha \phi\left[1-\frac{1}{R}\right]}{c^{\prime} \mu\left[1-\frac{1}{R^{\prime}}\right]}$, como definido no lema 3.2.2. Segue, então, o lema:

Lema 3.3.9 $O$ ponto $\left(P_{3}, M_{3}, L_{3}, G_{3}\right)$, dado por (3.8), tem indice de estabilidade:

(a) $\sigma=\mathbf{3}$ se $R^{\prime}>1$ e $k<k_{\max }$,

(a) $\sigma=4$ se $R^{\prime}>1$ e $k>k_{\text {max }}$,

$\left(\mathrm{b}_{1}\right) \sigma=\mathbf{2}$ se $R^{\prime}<1$ ek> $k_{\text {max }}$,

$\left(\mathrm{b}_{2}\right) \sigma=\mathbf{3}$ se $R^{\prime}<1$ e $k<k_{\text {max }}$.

\section{Demonstração 3.3.9 Seja}

$$
\Delta=\left(\alpha+\beta-\mu+c^{\prime} k\left[1-\frac{1}{R^{\prime}}\right]\right)^{2}+4 \alpha \phi
$$

a expressão que aparece dentro do radical de $\lambda_{1}$ e $\lambda_{2}$, dadas em (3.16).

Como $\Delta>0, \lambda_{1}$ e $\lambda_{2}$ são ambos reais e $\lambda_{2}<0$. Analisemos, então, os outros três autovalores. 
Note que a expressão para $\Delta$ pode ser escrita, como em (3.17), na forma:

$$
\begin{aligned}
\Delta & =\left(\alpha+\beta-\mu+c^{\prime} k\left[1-\frac{1}{R^{\prime}}\right]\right)^{2}+4 \alpha \phi \\
& =\left(\alpha+\beta+\mu+c^{\prime}\left[1-\frac{1}{R^{\prime}}\right] k\right)^{2}-4\left[\mu\left(\alpha+\beta+c^{\prime}\left[1-\frac{1}{R^{\prime}}\right] k\right)-\alpha \phi\right] \\
& =\left(\alpha+\beta+\mu+c^{\prime}\left[1-\frac{1}{R^{\prime}}\right] k\right)^{2}-4[\mu(\alpha+\beta)-\alpha \phi]-4 k \mu c^{\prime}\left[1-\frac{1}{R^{\prime}}\right] \\
& =\left(\alpha+\beta+\mu+c^{\prime}\left[1-\frac{1}{R^{\prime}}\right] k\right)^{2}+4 \alpha \phi\left[1-\frac{1}{R}\right]-4 k \mu c^{\prime}\left[1-\frac{1}{R^{\prime}}\right] .
\end{aligned}
$$

Concluímos, então, por esta última expressão, que:

$$
4 \alpha \phi\left[1-\frac{1}{R}\right]-4 k \mu c^{\prime}\left[1-\frac{1}{R^{\prime}}\right]>0 \Rightarrow \lambda_{1}>0
$$

$e$

$$
4 \alpha \phi\left[1-\frac{1}{R}\right]-4 k \mu c^{\prime}\left[1-\frac{1}{R^{\prime}}\right]<0 \Rightarrow \lambda_{1}<0 .
$$

(a) Se $R^{\prime}>1$, então $-4 \alpha^{\prime} \phi^{\prime}\left[1-\frac{1}{R^{\prime}}\right]<0$. Como essa expressão aparece dentro do radical de $\lambda_{3}$ e $\lambda_{4}$, dadas em (3.16), temos que $\lambda_{3}$ e $\lambda_{4}$ são complexos, não reais, com parte real negativa, ou são ambos reais e negativos.

(a $\left.\mathbf{a}_{1}\right)$ Se $k<k_{m a x}$, então $\alpha \phi\left[1-\frac{1}{R}\right]>k \mu c^{\prime}\left[1-\frac{1}{R^{\prime}}\right]$ e, por consequência, vale a relação (3.18). Portanto temos $\lambda_{1}>0$ e $\lambda_{3}, \lambda_{4}$ ambos com parte real negativa.

(a $\left.\mathbf{a}_{2}\right)$ Se $k>k_{\text {max }}$, então $\alpha \phi\left[1-\frac{1}{R}\right]<k \mu c^{\prime}\left[1-\frac{1}{R^{\prime}}\right]$ e vale a relação (3.19). Portanto temos $\lambda_{1}<0$ e $\lambda_{3}, \lambda_{4}$ ambos com parte real negativa.

(b) Se $R^{\prime}<1$, então $-4 \alpha^{\prime} \phi^{\prime}\left[1-\frac{1}{R^{\prime}}\right]>0$, implicando que a expressão dentro do radical de $\lambda_{3}$ e $\lambda_{4}$, dadas em (3.16), é positiva. Temos, portanto, $\lambda_{3}>0$ e $\lambda_{4}<0$

( $\left.\mathrm{b}_{1}\right)$ Se $k>k_{\text {max }}$, então $\alpha \phi\left[1-\frac{1}{R}\right]>k \mu c^{\prime}\left[1-\frac{1}{R^{\prime}}\right]$ e, por consequência, vale a relação (3.18). Portanto temos $\lambda_{1}>0, \lambda_{3}>0, \lambda_{4}<0$ todos reais.

$\left(\mathrm{b}_{2}\right)$ Se $k<k_{\max }$, então $\alpha \phi\left[1-\frac{1}{R}\right]<k \mu c^{\prime}\left[1-\frac{1}{R^{\prime}}\right]$, valendo a relação (3.19). Portanto temos $\lambda_{1}<0, \lambda_{3}>0, \lambda_{4}<0$ todos reais. 
Lema 3.3.10 $\lambda_{3}$ e $\lambda_{4}$, dados por (3.16), são:

(a) complexos conjugados, não reais, se $\phi^{\prime}>\frac{1}{4 \alpha^{\prime}}\left[\left(\alpha^{\prime}+\beta^{\prime}+\mu^{\prime}\right)^{2}+4 \mu^{\prime}\left(\alpha^{\prime}+\beta^{\prime}\right)\right]$, ou

(b) reais, se $\phi^{\prime} \leq \frac{1}{4 \alpha^{\prime}}\left[\left(\alpha^{\prime}+\beta^{\prime}+\mu^{\prime}\right)^{2}+4 \mu^{\prime}\left(\alpha^{\prime}+\beta^{\prime}\right)\right]$.

\section{Demonstração 3.3.10 Seja}

$$
\begin{aligned}
\Delta & =\left(\alpha^{\prime}+\beta^{\prime}+\mu^{\prime}\right)^{2}-4 \alpha^{\prime} \phi^{\prime}\left[1-\frac{1}{R^{\prime}}\right] \\
& =\left(\alpha^{\prime}+\beta^{\prime}+\mu^{\prime}\right)^{2}+4 \mu^{\prime}\left(\alpha^{\prime}+\beta^{\prime}\right)-4 \alpha^{\prime} \phi^{\prime}
\end{aligned}
$$

a expressão que aparece dentro do radical de $\lambda_{3}$ e $\lambda_{4}$ em (3.16).

Temos que:

(a) se $\phi^{\prime}>\frac{1}{4 \alpha^{\prime}}\left[\left(\alpha^{\prime}+\beta^{\prime}+\mu^{\prime}\right)^{2}+4 \mu^{\prime}\left(\alpha^{\prime}+\beta^{\prime}\right)\right] \Rightarrow \Delta<0 \Rightarrow$ por (3.16), $\lambda_{3}$ e $\lambda_{4}$ são complexos conjugados, não reais.

(b) se $\phi^{\prime} \leq \frac{1}{4 \alpha^{\prime}}\left[\left(\alpha^{\prime}+\beta^{\prime}+\mu^{\prime}\right)^{2}+4 \mu^{\prime}\left(\alpha^{\prime}+\beta^{\prime}\right)\right] \Rightarrow \Delta \geq 0 \Rightarrow$ por (3.16), $\lambda_{3}$ e $\lambda_{4}$ são reais.

\subsection{4 $\left(\mathrm{P}_{4}, \mathrm{M}_{4}, \mathrm{~L}_{4}, \mathrm{G}_{4}\right)$}

Quanto ao ponto $x=\left(P_{4}, M_{4}, L_{4}, G_{4}\right)$, dado por (3.9), não conseguiremos prosseguir da mesma maneira pois sua equação característica não é facilmente fatorável. Vejamos:

A matriz Jacobiana de (S) é dada por

$$
J(x)=\left(\begin{array}{cccc}
-\alpha-\beta-k G & \phi-\frac{2 \phi M}{c} & 0 & -k P \\
\alpha & -\mu & 0 & 0 \\
k^{\prime} G & 0 & -\alpha^{\prime}-\beta^{\prime} & \phi^{\prime}-\frac{2 \phi^{\prime} G}{c^{\prime}}+k^{\prime} P \\
0 & 0 & \alpha^{\prime} & -\mu^{\prime}
\end{array}\right)
$$

Para calcularmos sua equação característica fazemos $\operatorname{det}(J(x)-\lambda I)=0$, onde $I$ representa 
a matriz identidade, obtendo assim:

$$
\begin{gathered}
{\left[(\mu+\lambda)(\alpha+\beta+k G+\lambda)-\alpha\left(\phi-2 \frac{\phi M}{c}\right)\right]\left[\left(\mu^{\prime}+\lambda\right)\left(\alpha^{\prime}+\beta^{\prime}+\lambda\right)-\alpha^{\prime}\left(\phi^{\prime}-2 \frac{\phi^{\prime} G}{c^{\prime}}+k^{\prime} P\right)\right]} \\
+\alpha^{\prime}(\mu+\lambda) k k^{\prime} P G=0 .
\end{gathered}
$$

Note que, para os três primeiros pontos, $\left(P_{1}, M_{1}, L_{1}, G_{1}\right),\left(P_{2}, M_{2}, L_{2}, G_{2}\right)$ e $\left(P_{3}, M_{3}, L_{3}, G_{3}\right)$, a equação característica (3.20) fica fatorada, pois o único termo externo aos colchetes nesta equação se anula, pois, ou $P$, ou $G$ ou ambos são nulos. Já o ponto $\left(P_{4}, M_{4}, L_{4}, G_{4}\right)$, dado por (3.9), não anula esse termo.

Lema 3.3.11 A equação característica (3.20), calculada em $\left(P_{4}, M_{4}, L_{4}, G_{4}\right)$, pode ser escrita na forma

$$
\lambda^{4}+\tilde{b} \lambda^{3}+\tilde{c} \lambda^{2}+\tilde{d} \lambda+\tilde{e}=0
$$

$\operatorname{com} \tilde{b}, \tilde{c}, \tilde{d} e \tilde{e}$ dados por:

$$
\begin{aligned}
& \tilde{b}=\alpha+\beta+\mu+\alpha^{\prime}+\beta^{\prime}+\mu^{\prime}+k G_{4}, \\
& \tilde{c}=\frac{\alpha \phi}{c} M_{4}+\frac{\alpha^{\prime} \phi^{\prime}}{c^{\prime}} G_{4}+\left(\alpha+\beta+\mu+k G_{4}\right)\left(\alpha^{\prime}+\beta^{\prime}+\mu^{\prime}\right), \\
& \tilde{d}=\left(\alpha+\beta+\mu+k G_{4}\right) \frac{\alpha^{\prime} \phi^{\prime}}{c^{\prime}} G_{4}+\left(\alpha^{\prime}+\beta^{\prime}+\mu^{\prime}\right) \frac{\alpha \phi}{c} M_{4}+\alpha^{\prime} k k^{\prime} P_{4} G_{4}, \\
& \tilde{e}=\alpha^{\prime} \alpha \phi\left(k^{\prime}\left[1-\frac{1}{R}\right] P_{4}+\frac{\phi^{\prime}}{c}\left[1-\frac{1}{R^{\prime}}\right] M_{4}\right) .
\end{aligned}
$$

Demonstração 3.3.11 Primeiramente verifiquemos as relações seguintes:

$$
\begin{gathered}
\alpha \phi\left[1-\frac{1}{R}\right]-\mu k G_{4}=\frac{\alpha \phi}{c} M_{4}, \\
\alpha^{\prime} \phi^{\prime}\left[1-\frac{1}{R^{\prime}}\right]+\alpha^{\prime} k^{\prime} P_{4}=\frac{\alpha^{\prime} \phi^{\prime}}{c^{\prime}} G_{4} .
\end{gathered}
$$


De fato,

$$
\begin{aligned}
& \alpha \phi\left[1-\frac{1}{R}\right]-\mu k G_{4}= \\
& =\alpha \phi\left[1-\frac{1}{R}\right]-\frac{\mu k c^{\prime} \alpha \phi}{\alpha^{2} \phi \phi^{\prime}+\mu^{2} c c^{\prime} k k^{\prime}}\left(c \mu k^{\prime}\left[1-\frac{1}{R}\right]+\alpha \phi^{\prime}\left[1-\frac{1}{R^{\prime}}\right]\right) \\
& =\left(\alpha \phi\left(\alpha^{2} \phi \phi^{\prime}+\mu^{2} c c^{\prime} k k^{\prime}\right)\left[1-\frac{1}{R}\right]-\mu k c^{\prime} \alpha \phi\left(c \mu k^{\prime}\left[1-\frac{1}{R}\right]+\alpha \phi^{\prime}\left[1-\frac{1}{R^{\prime}}\right]\right)\right) \frac{1}{\alpha^{2} \phi \phi^{\prime}+\mu^{2} c c^{\prime} k k^{\prime}} \\
& =\left(\alpha^{3} \phi^{2} \phi^{\prime}\left[1-\frac{1}{R}\right]+\mu k c^{\prime} \alpha^{2} \phi \phi^{\prime}\left[1-\frac{1}{R^{\prime}}\right]\right) \frac{1}{\alpha^{2} \phi \phi^{\prime}+\mu^{2} c c^{\prime} k k^{\prime}} \\
& =\alpha^{2} \phi \phi^{\prime}\left(\alpha \phi\left[1-\frac{1}{R}\right]+\mu k c^{\prime}\left[1-\frac{1}{R^{\prime}}\right]\right) \frac{1}{\alpha^{2} \phi \phi^{\prime}+\mu^{2} c c^{\prime} k k^{\prime}} \\
& =\frac{\alpha \phi}{c} M_{4}
\end{aligned}
$$

o que prova a relação (3.21), e

$$
\begin{aligned}
& \alpha^{\prime} \phi^{\prime}\left[1-\frac{1}{R^{\prime}}\right]+\alpha^{\prime} k^{\prime} P_{4}= \\
& =\alpha^{\prime} \phi^{\prime}\left[1-\frac{1}{R^{\prime}}\right]+\frac{\alpha^{\prime} k^{\prime} c \mu \phi^{\prime}}{\alpha^{2} \phi \phi^{\prime}+\mu^{2} c c^{\prime} k k^{\prime}}\left(\alpha \phi\left[1-\frac{1}{R}\right]-\mu c^{\prime} k\left[1-\frac{1}{R^{\prime}}\right]\right) \\
& =\left[\alpha^{\prime} \phi^{\prime}\left(\alpha^{2} \phi \phi^{\prime}+\mu^{2} c c^{\prime} k k^{\prime}\right)\left[1-\frac{1}{R^{\prime}}\right]+\alpha^{\prime} k^{\prime} c \mu \phi^{\prime}\left(\alpha \phi\left[1-\frac{1}{R}\right]-\mu c^{\prime} k\left[1-\frac{1}{R^{\prime}}\right]\right)\right] \frac{1}{\alpha^{2} \phi \phi^{\prime}+\mu^{2} c c^{\prime} k k^{\prime}} \\
& =\left[\alpha^{\prime} \phi^{\prime 2} \alpha^{2} \phi\left[1-\frac{1}{R^{\prime}}\right]+\alpha^{\prime} k^{\prime} c \mu \phi^{\prime} \alpha \phi\left[1-\frac{1}{R}\right] \frac{1}{\alpha^{2} \phi \phi^{\prime}+\mu^{2} c c^{\prime} k k^{\prime}}\right. \\
& =\alpha^{\prime} \phi^{\prime} \alpha \phi\left(\alpha \phi^{\prime}\left[1-\frac{1}{R^{\prime}}\right]+k^{\prime} c \mu\left[1-\frac{1}{R}\right]\right) \frac{1}{\alpha^{2} \phi \phi^{\prime}+\mu^{2} c c^{\prime} k k^{\prime}} \\
& =\frac{\alpha^{\prime} \phi^{\prime}}{c^{\prime}} G_{4}
\end{aligned}
$$

provando a relação (3.22).

Consideremos agora a equação (3.20), calculada em $\left(P_{4}, M_{4}, L_{4}, G_{4}\right)$,

$$
\begin{gathered}
{\left[(\mu+\lambda)\left(\alpha+\beta+k G_{4}+\lambda\right)-\alpha\left(\phi-2 \frac{\phi M_{4}}{c}\right)\right]\left[\left(\mu^{\prime}+\lambda\right)\left(\alpha^{\prime}+\beta^{\prime}+\lambda\right)-\alpha^{\prime}\left(\phi^{\prime}-2 \frac{\phi^{\prime} G_{4}}{c^{\prime}}+k^{\prime} P_{4}\right)\right]} \\
+\alpha^{\prime}(\mu+\lambda) k k^{\prime} P_{4} G_{4}=0 .
\end{gathered}
$$

Note que a expressão

$$
\begin{gathered}
(\mu+\lambda)\left(\alpha+\beta+k G_{4}+\lambda\right)-\alpha\left(\phi-2 \frac{\phi M_{4}}{c}\right)= \\
=\lambda^{2}+\left(\alpha+\beta+\mu+k G_{4}\right) \lambda+\mu(\alpha+\beta)+\mu k G_{4}-\alpha \phi+2 \frac{\alpha \phi}{c} M_{4} \\
=\lambda^{2}+\left(\alpha+\beta+\mu+k G_{4}\right) \lambda-\alpha \phi\left[1-\frac{1}{R}\right]+\mu k G_{4}+2 \frac{\alpha \phi}{c} M_{4},
\end{gathered}
$$

e, devido à relação (3.21), fica dada por

$$
\lambda^{2}+\left(\alpha+\beta+\mu+k G_{4}\right) \lambda+\frac{\alpha \phi}{c} M_{4}
$$


Note também que a expressão

$$
\begin{gathered}
\left(\mu^{\prime}+\lambda\right)\left(\alpha^{\prime}+\beta^{\prime}+\lambda\right)-\alpha^{\prime}\left(\phi^{\prime}-2 \frac{\phi^{\prime} G_{4}}{c^{\prime}}+k^{\prime} P_{4}\right) \\
=\lambda^{2}+\left(\alpha^{\prime}+\beta^{\prime}+\mu^{\prime}\right) \lambda+\mu^{\prime}\left(\alpha^{\prime}+\beta^{\prime}\right)-\alpha^{\prime} \phi^{\prime}+2 \frac{\alpha^{\prime} \phi^{\prime}}{c^{\prime}} G_{4}-\alpha^{\prime} k^{\prime} P_{4} \\
=\lambda^{2}+\left(\alpha^{\prime}+\beta^{\prime}+\mu^{\prime}\right) \lambda-\alpha^{\prime} \phi^{\prime}\left[1-\frac{1}{R^{\prime}}\right]-\alpha^{\prime} k^{\prime} P_{4}+2 \frac{\alpha^{\prime} \phi^{\prime}}{c^{\prime}} G_{4},
\end{gathered}
$$

fica dada, devida a relação (3.22), por

$$
\lambda^{2}+\left(\alpha^{\prime}+\beta^{\prime}+\mu^{\prime}\right) \lambda+\frac{\alpha^{\prime} \phi^{\prime}}{c^{\prime}} G_{4}
$$

Dessa forma, temos a equação considerada escrita como

$$
\begin{gathered}
{\left[\lambda^{2}+\left(\alpha+\beta+\mu+k G_{4}\right) \lambda+\frac{\alpha \phi}{c} M_{4}\right]\left[\lambda^{2}+\left(\alpha^{\prime}+\beta^{\prime}+\mu^{\prime}\right) \lambda+\frac{\alpha^{\prime} \phi^{\prime}}{c^{\prime}} G_{4}\right]} \\
+\alpha^{\prime} \mu k k^{\prime} P_{4} G_{4}+\alpha^{\prime} k k^{\prime} P_{4} G_{4} \lambda=0
\end{gathered}
$$

que, aplicando os produtos, obtemos

$$
\begin{aligned}
& \lambda^{4}+\lambda^{3}\left[\alpha+\beta+\mu+\alpha^{\prime}+\beta^{\prime}+\mu^{\prime}+k G_{4}\right] \\
& +\lambda^{2}\left[\frac{\alpha \phi}{c} M_{4}+\frac{\alpha^{\prime} \phi^{\prime}}{c^{\prime}} G_{4}+\left(\alpha+\beta+\mu+k G_{4}\right)\left(\alpha^{\prime}+\beta^{\prime}+\mu^{\prime}\right)\right] \\
& +\lambda\left[\left(\alpha+\beta+\mu+k G_{4}\right) \frac{\alpha^{\prime} \phi^{\prime}}{c^{\prime}} G_{4}+\left(\alpha^{\prime}+\beta^{\prime}+\mu^{\prime}\right) \frac{\alpha \phi}{c} M_{4}+\alpha^{\prime} k k^{\prime} P_{4} G_{4}\right] \\
& +\frac{\alpha \phi}{c} M_{4} \frac{\alpha^{\prime} \phi^{\prime}}{c^{\prime}} G_{4}+\alpha^{\prime} \mu k k^{\prime} P_{4} G_{4}=0 .
\end{aligned}
$$

Portanto,

$$
\begin{aligned}
\tilde{b} & =\alpha+\beta+\mu+\alpha^{\prime}+\beta^{\prime}+\mu^{\prime}+k G_{4}, \\
\tilde{c} & =\frac{\alpha \phi}{c} M_{4}+\frac{\alpha^{\prime} \phi^{\prime}}{c^{\prime}} G_{4}+\left(\alpha+\beta+\mu+k G_{4}\right)\left(\alpha^{\prime}+\beta^{\prime}+\mu^{\prime}\right), \\
\tilde{d} & =\left(\alpha+\beta+\mu+k G_{4}\right) \frac{\alpha^{\prime} \phi^{\prime}}{c^{\prime}} G_{4}+\left(\alpha^{\prime}+\beta^{\prime}+\mu^{\prime}\right) \frac{\alpha \phi}{c} M_{4}+\alpha^{\prime} k k^{\prime} P_{4} G_{4}, \\
\tilde{e} & =\frac{\alpha \phi}{c} M_{4} \frac{\alpha^{\prime} \phi^{\prime}}{c^{\prime}} G_{4}+\alpha^{\prime} \mu k k^{\prime} P_{4} G_{4} \\
& =\left(\alpha \phi\left[1-\frac{1}{R}\right]-\mu k G_{4}\right)\left(\alpha^{\prime} \phi^{\prime}\left[1-\frac{1}{R^{\prime}}\right]+\alpha^{\prime} k^{\prime} P_{4}\right)+\alpha^{\prime} \mu k k^{\prime} P_{4} G_{4} \\
& =\alpha \phi\left[1-\frac{1}{R}\right] \alpha^{\prime} \phi^{\prime}\left[1-\frac{1}{R^{\prime}}\right]+\alpha^{\prime} k^{\prime} P_{4} \alpha \phi\left[1-\frac{1}{R}\right]-\mu k G_{4} \alpha^{\prime} \phi^{\prime}\left[1-\frac{1}{R^{\prime}}\right] \\
& =\alpha \phi\left[1-\frac{1}{R}\right] \alpha^{\prime} \phi^{\prime}\left[1-\frac{1}{R^{\prime}}\right]+\alpha^{\prime} k^{\prime} P_{4} \alpha \phi\left[1-\frac{1}{R}\right]+\alpha^{\prime} \phi^{\prime}\left[1-\frac{1}{R^{\prime}}\right]\left(\frac{\alpha \phi}{c} M_{4}-\alpha \phi\left[1-\frac{1}{R}\right]\right) \\
& =\alpha^{\prime} k^{\prime} \alpha \phi\left[1-\frac{1}{R}\right] P_{4}+\alpha^{\prime} \phi^{\prime}\left[1-\frac{1}{R^{\prime}} \frac{\alpha \phi}{c} M_{4}\right. \\
& =\alpha^{\prime} \alpha \phi\left(k^{\prime}\left[1-\frac{1}{R}\right] P_{4}+\frac{\phi^{\prime}}{c}\left[1-\frac{1}{R^{\prime}}\right] M_{4}\right) .
\end{aligned}
$$


Para obtermos essa última expressão de ẽ utilizamos as relações (3.21) e (3.2Q).

Observação 3.3.1 Satisfeitas as hipóteses do lema 3.2.2 e, portanto, tendo $P_{4}>0, M_{4}>0$, $L_{4}>0, G_{4}>0$, os coeficientes $\tilde{b}, \tilde{c}, \tilde{d}$ e $\tilde{e}$ são todos claramente positivos.

O que faremos, então, será estudar a possibilidade deste ponto $\left(P_{4}, M_{4}, L_{4}, G_{4}\right)$ ser um ponto de Andronov-Hopf.

\subsection{Condição de Hopf}

Como acabamos de ver, a matriz Jacobiana de (S), dada por

$$
J(x)=\left(\begin{array}{cccc}
-\alpha-\beta-k G & \phi-\frac{2 \phi M}{c} & 0 & -k P \\
\alpha & -\mu & 0 & 0 \\
k^{\prime} G & 0 & -\alpha^{\prime}-\beta^{\prime} & \phi^{\prime}-\frac{2 \phi^{\prime} G}{c^{\prime}}+k^{\prime} P \\
0 & 0 & \alpha^{\prime} & -\mu^{\prime}
\end{array}\right)
$$

se calculada no ponto $\left(P_{4}, M_{4}, L_{4}, G_{4}\right)$, possui equação característica do quarto grau

$$
\lambda^{4}+\tilde{b} \lambda^{3}+\tilde{c} \lambda^{2}+\tilde{d} \lambda+\tilde{e}=0
$$

sendo:

$$
\begin{aligned}
& \tilde{b}=\alpha+\beta+\mu+\alpha^{\prime}+\beta^{\prime}+\mu^{\prime}+k G_{4}, \\
& \tilde{c}=\frac{\alpha \phi}{c} M_{4}+\frac{\alpha^{\prime} \phi^{\prime}}{c^{\prime}} G_{4}+\left(\alpha+\beta+\mu+k G_{4}\right)\left(\alpha^{\prime}+\beta^{\prime}+\mu^{\prime}\right), \\
& \tilde{d}=\left(\alpha+\beta+\mu+k G_{4}\right) \frac{\alpha^{\prime} \phi^{\prime}}{c^{\prime}} G_{4}+\left(\alpha^{\prime}+\beta^{\prime}+\mu^{\prime}\right) \frac{\alpha \phi}{c} M_{4}+\alpha^{\prime} k k^{\prime} P_{4} G_{4}, \\
& \tilde{e}=\alpha^{\prime} \alpha \phi\left(k^{\prime}\left[1-\frac{1}{R}\right] P_{4}+\frac{\phi^{\prime}}{c}\left[1-\frac{1}{R^{\prime}}\right] M_{4}\right) .
\end{aligned}
$$

Lema 3.4.1 Se considerarmos $\tilde{b}, \tilde{c}, \tilde{d}, \tilde{e}>0$, a matriz Jacobiana do sistema (S) possuirá um par de autovalores imaginários puros se e só se

$$
\tilde{d}^{2}-\tilde{b} \tilde{c} \tilde{d}+\tilde{e} \tilde{b}^{2}=0
$$


Demonstração 3.4.1 Sejam $\lambda_{1}$ e $\lambda_{2}$ raízes da equação característica

$$
\lambda^{4}+\tilde{b} \lambda^{3}+\tilde{c} \lambda^{2}+\tilde{d} \lambda+\tilde{e}=0
$$

Se $\lambda_{1}$ é imaginário puro, $\lambda_{1}=\eta i(\eta \in \mathbb{R})$, então $\lambda_{2}=\overline{\lambda_{1}}=-\eta i$ também é raiz pois $\tilde{b}, \tilde{c}, \tilde{d}, \tilde{e} \in \mathbb{R}$. Substituindo $\lambda_{1}$ na equação característica, vem então que

$$
\begin{gathered}
\eta^{4}-\eta^{3} \tilde{b} i-\eta^{2} \tilde{c}+\eta \tilde{d} i+\tilde{e}=0 \\
\left(\eta^{4}-\eta^{2} \tilde{c}+\tilde{e}\right)+i\left(-\eta^{3} \tilde{b}+\eta \tilde{d}\right)=0 \\
\Leftrightarrow\left\{\begin{array}{l}
\eta^{4}-\eta^{2} \tilde{c}+\tilde{e}=0 \\
-\eta^{3} \tilde{b}+\eta \tilde{d}=0
\end{array}\right. \\
\Leftrightarrow\left\{\begin{array}{l}
\eta^{4}-\eta^{2} \tilde{c}+\tilde{e}=0 \\
\eta^{2} \tilde{b}-\tilde{d}=0
\end{array}\right.
\end{gathered}
$$

Da segunda equação em (3.23) temos

$$
\eta^{2}=\frac{\tilde{d}}{\tilde{b}}
$$

Substituindo (3.24) na primeira equação do sistema (3.23), obtemos:

$$
\begin{aligned}
& \frac{\tilde{d}^{2}}{\tilde{b}^{2}}-\frac{\tilde{d}}{\tilde{b}} \tilde{c}+\tilde{e}=0 \\
\Leftrightarrow & \tilde{d}^{2}-\tilde{b} \tilde{c} \tilde{d}+\tilde{e} \tilde{b}^{2}=0 .
\end{aligned}
$$

Para simplificar a notação, não mencionamos aqui a dependência dos coeficientes $\tilde{b}, \tilde{c}, \tilde{d}, \tilde{e}$ com relação ao parâmetro de bifurcação. Como os parâmetros $k$ e $k^{\prime}$, descritos no capítulo 1, são exatamente os parâmetros que evidenciam a interação entre as espécies, tomaremos estes como os parâmetros para estudar a bifurcação. Desse modo o que faremos será fixar $k^{\prime}$ e procurar $k$ que satisfaça o lema anterior. Portanto devemos considerar $\tilde{b}=\tilde{b}(k), \tilde{c}=\tilde{c}(k)$, $\tilde{d}=\tilde{d}(k)$ e $\tilde{e}=\tilde{e}(k)$. 


\subsection{Resumo}

No estudo feito na seção 3.3, concluímos que a matriz Jacobiana do sistema (S) no ponto de equilíbrio $\left(P_{1}, M_{1}, L_{1}, G_{1}\right)$ possui autovalores que não dependem dos parâmetros $k$ e $k^{\prime}$, veja (3.14). Concluímos também que para $R>1$, situação essa que verifica a permanência do ponto $\left(P_{2}, M_{2}, L_{2}, G_{2}\right)$, dado por (3.7), no "quadrante" positivo, os autovalores $\lambda_{3}$ e $\lambda_{4}$, dados em (3.15), que dependem de $k^{\prime}$, são sempre reais (lema 3.3.5). Por fim, para o ponto $\left(P_{3}, M_{3}, L_{3}, G_{3}\right)$, dado por (3.8), concluímos que os seus autovalores $\lambda_{1}$ e $\lambda_{2}$, dados em (3.16), que dependem de $k$, são também reais (veja o início da demonstração do lema 3.3.9). Em outras palavras, o que queremos dizer é que não há possibilidade dos pontos de equilíbrio $\left(P_{1}, M_{1}, L_{1}, G_{1}\right),\left(P_{2}, M_{2}, L_{2}, G_{2}\right)$ e $\left(P_{3}, M_{3}, L_{3}, G_{3}\right)$ serem pontos de Hopf com relação aos parâmetros $k$ ou $k^{\prime}$ e possuírem uma interpretação biológica. $\mathrm{O}$ único ponto de equilíbrio que poderá apresentar esse comportamento será o ponto $\left(P_{4}, M_{4}, L_{4}, G_{4}\right)$.

Lembramos que a existência de um par de autovalores puramente imaginários é essencial mas não garante que o ponto seja de Hopf. Precisamos também verificar as condições

(1) $l_{1}(0) \neq 0$ (não degenerescência) e

(2) $\gamma^{\prime}(0) \neq 0$ (transversalidade),

vistas em (2.1.1).

Portanto, estando satisfeitas as condições

$$
R>1, R^{\prime}>1, k<k_{\max }
$$

do ítem (a), no lema 3.2.2, pág. 61, ficam satisfeitas também as condições (a) (ou (a $\mathbf{a}_{1}$ ), quando (a) é subdividida em $\left(a_{1}\right),\left(a_{2}\right)$, etc...) dos lemas: 3.3 .3 , pág. 65; 3.3.6, pág. 68 e 3.3.9, pág. 71. Se $k$ ainda satisfizer o lema 3.4.1 e as condições (1) e (2) listadas no parágrafo anterior, o sistema (S) se comportará conforme a figura (3.2), onde o eixo horizontal representa o plano $P M$ e o eixo vertical o plano $L G$.

Devido às dificuldades na manipulação algébrica das expressões dos autovalores da matriz Jacobiana no ponto $\left(P_{4}, M_{4}, L_{4}, G_{4}\right)$, dado o número excessivo de parâmetros na equação quártica que representa o polinômio característico, prosseguiremos nosso estudo, no capítulo 4, com o auxílio de simulações numéricas. 


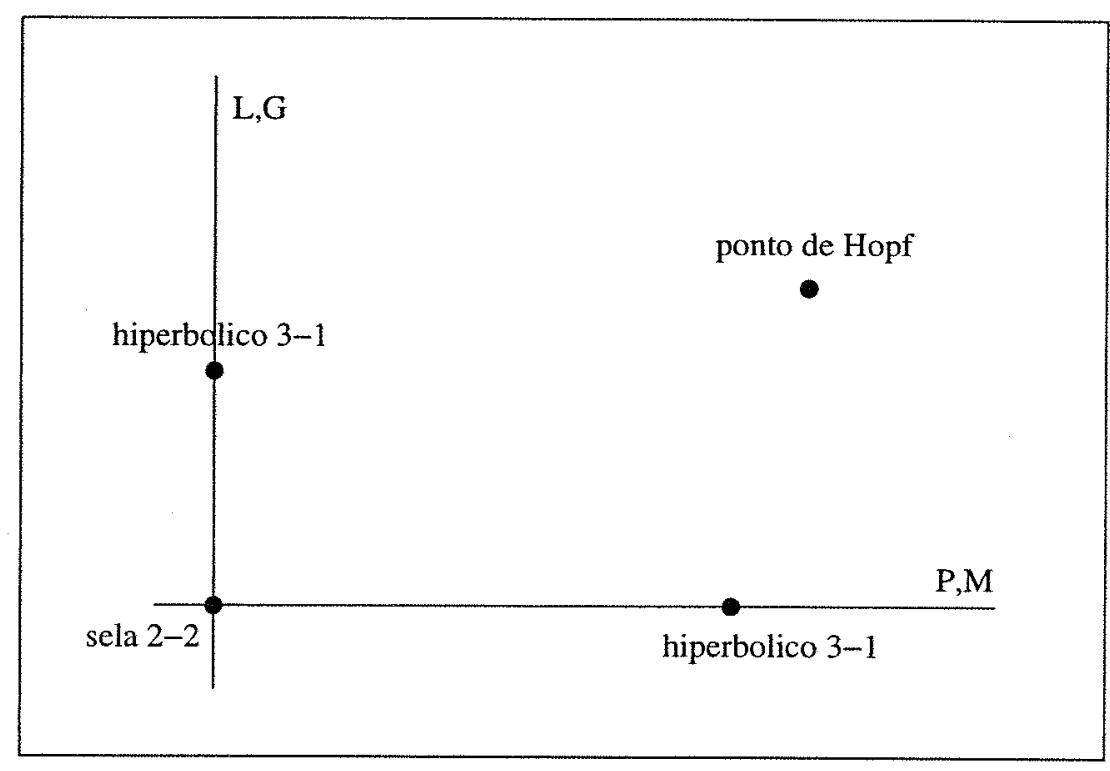

Figura 3.2: Retrato $P M \times L G$ 


\section{Capítulo 4}

\section{Simulações Numéricas}

Temos por objetivo, neste capítulo, verificar numericamente a existência de uma bifurcação de Hopf no modelo de interação biológica proposto no capítulo 1 com relação aos parâmetros $k$ e $k^{\prime}$, responsáveis pela interação das espécies. Para tanto utilizaremos os resultados descritos nos capítulos anteriores, fixando $k^{\prime}$ e procurando encontrar o valor de $k$ no qual ocorre a bifurcação.

Todos os cálculos e rotinas que usamos na obtenção dos resultados aqui apresentados foram feitos no Maple V. Para os parâmetros relacionados à praga, $\alpha, \beta, \mu, \phi, c$ e $k$, utilizamos no Maple a notação alpha1, beta1, mu1, phi1, c1 e $k 1(\alpha 1, \beta 1, \mu 1, \phi 1, c 1$ e $k 1)$ e, para os parâmetros relacionados ao parasito, $\alpha^{\prime}, \beta^{\prime}, \mu^{\prime}, \phi^{\prime}, c^{\prime}$ e $k^{\prime}$, utilizamos no Maple

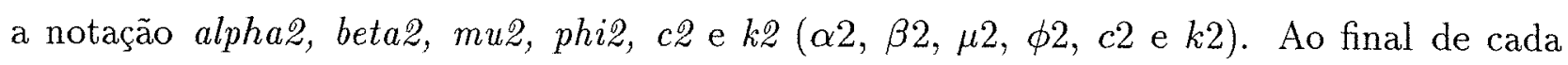
seção ou subseção, apresentaremos, na forma de tabelas, os comandos do Maple utilizados para a obtenção dos resultados aqui expostos. Se necessário, juntamente indicaremos outras alterações na notação. Apesar de divididos em tabelas diferentes, os comandos formam uma sequência única, de modo que, muito provavelmente, as rotinas apresentadas nas tabelas últimas não funcionarão, se implementadas, sem as apresentadas nas primeiras tabelas e na mesma sequência, ou seja, para que essas rotinas cumpram seus objetivos é necessário que sejam implementadas na sequência em que são aqui apresentadas. 


\subsection{Estudo de um caso específico}

Faremos aqui um estudo do comportamento das soluções do sistema

$$
(\mathbf{S})\left\{\begin{array}{l}
\frac{d P}{d t}=\phi\left(1-\frac{M}{c}\right) M-(\alpha+\beta) P-k P G \\
\frac{d M}{d t}=\alpha P-\mu M \\
\frac{d L}{d t}=\phi^{\prime}\left(1-\frac{G}{c^{\prime}}\right) G-\left(\alpha^{\prime}+\beta^{\prime}\right) L+k^{\prime} P G \\
\frac{d G}{d t}=\alpha^{\prime} L-\mu^{\prime} G
\end{array}\right.
$$

para um determinado conjunto de dados. Segue abaixo os valores que serão utilizados para os parâmetros:

$$
\begin{array}{lllll}
\alpha=.7 & \beta=.003 & \mu=.6 & \phi=2.3 & c=400000 \\
\alpha^{\prime}=.3 & \beta^{\prime}=.0015 & \mu^{\prime}=.4 & \phi^{\prime}=4 & c^{\prime}=100
\end{array}
$$

Todos estes valores, com exceção de $c$ e $c^{\prime}$ que possuem unidade insetos por talhão, como vimos no capítulo 1 , possuem dimensão dias ${ }^{-1}$ e foram propostos em Ternes [19].

\subsubsection{As equações desacopladas}

Analisaremos agora o sistema (S) com $k=k^{\prime}=0$. Primeiramente consideraremos o sistema. desacoplado para a população da praga $P$. citrella:

$$
(\mathbf{S}-\mathbf{1})\left\{\begin{array}{l}
\frac{d P}{d t}=\phi\left(1-\frac{M}{c}\right) M-(\alpha+\beta) P \\
\frac{d M}{d t}=\alpha P-\mu M
\end{array} .\right.
$$

De acordo com o lema 3.1.1, pág. 51, esse sistema tem dois pontos de equilíbrio dados por

$$
\begin{aligned}
& \left(P_{1}, M_{1}\right)=(0,0) e \\
& \left(P_{2}, M_{2}\right)=\left(\frac{\mu c}{\alpha}\left(1-\frac{1}{R}\right), c\left(1-\frac{1}{R}\right)\right),
\end{aligned}
$$

$\operatorname{com} R=\frac{\alpha \phi}{\mu(\alpha+\beta)}$. Substituindo, então, os valores dos parâmetros dados em (4.1), obtemos:

$$
\begin{aligned}
& R=3.816974869 \mathrm{e} \\
& \left(P_{2}, M_{2}\right)=(253032.8305,295204.9689)
\end{aligned}
$$


Portanto, segundo o lema 3.1.2, pág. 51, o ponto $\left(P_{1}, M_{1}\right)=(0,0)$ é um ponto de sela e, de acordo com o lema 3.1.3, pág. 55, o ponto $\left(P_{2}, M_{2}\right)$ é um foco atrator, uma vez que calculando o valor da expressão $\frac{1}{4 \alpha}\left[(\alpha+\beta+\mu)^{2}+4 \mu(\alpha+\beta)\right]$, obtemos

$$
\frac{1}{4 \alpha}\left[(\alpha+\beta+\mu)^{2}+4 \mu(\alpha+\beta)\right]=1.208931786
$$

valor este menor que $\phi=2.3$.

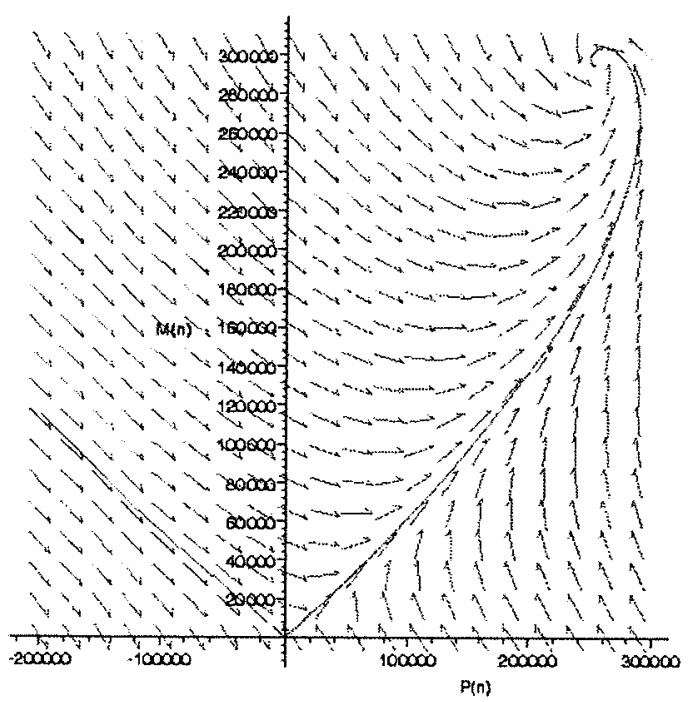

(a) visão global

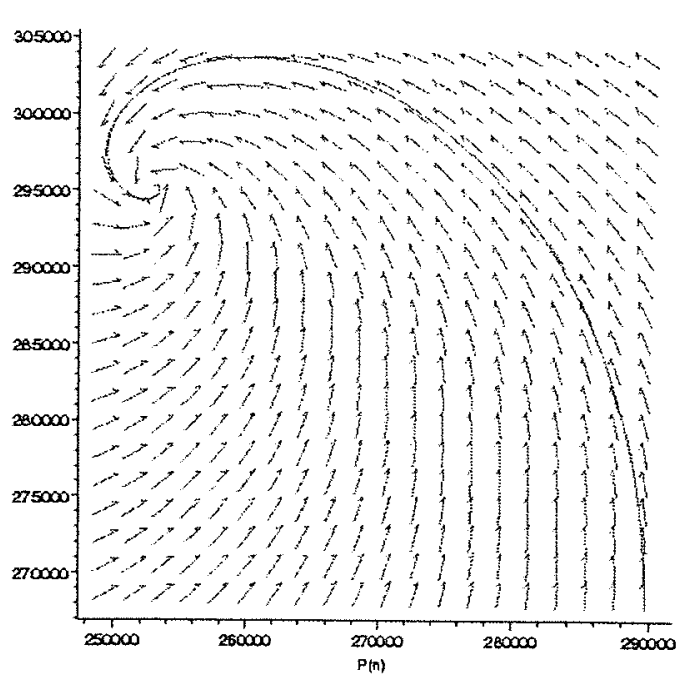

(b) visão ao redor de $\left(P_{2}, M_{2}\right)$

Figura 4.1: Retrato de fase $P \times M$

As figuras (4.1-a) e (4.1-b) representam os retratos de fase do sistema (S-1) para os parâmetros em questão, dados em (4.1). A linha contínua representa o comportamento da solução que, no instante inicial, passa pelo ponto $(P, M)=(5,20)$.

Da mesma forma analisaremos o sistema desacoplado para a população do parasito $G$. fausta:

$$
(\mathbf{S}-\mathbf{2})\left\{\begin{array}{l}
\frac{d L}{d t}=\phi^{\prime}\left(1-\frac{G}{c^{\prime}}\right) G-\left(\alpha^{\prime}+\beta^{\prime}\right) L \\
\frac{d G}{d t}=\alpha^{\prime} L-\mu^{\prime} G
\end{array} .\right.
$$




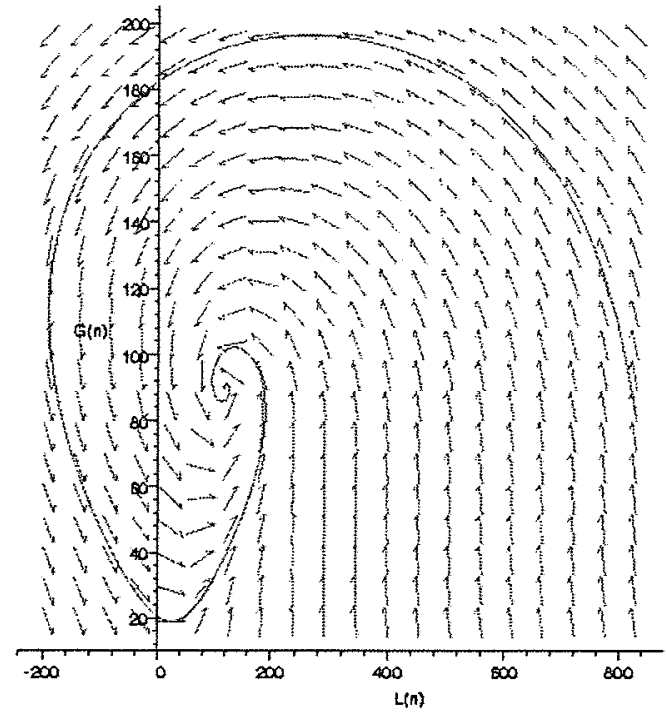

(a) visão global

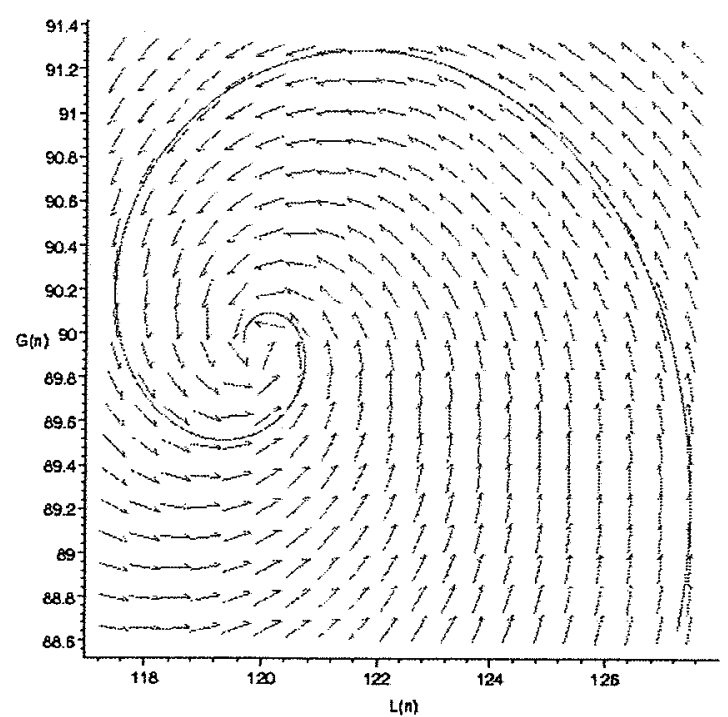

(b) visão ao redor de $\left(L_{2}, G_{2}\right)$

Figura 4.2: Retrato de fase $L \times G$

Esse sistema possui dois pontos de equilíbrio dados por

$$
\begin{aligned}
& \left(L_{1}, G_{1}\right)=(0,0) e \\
& \left(L_{2}, G_{2}\right)=\left(\frac{\mu^{\prime} c^{\prime}}{\alpha^{\prime}}\left(1-\frac{1}{R^{\prime}}\right), c^{\prime}\left(1-\frac{1}{R^{\prime}}\right)\right),
\end{aligned}
$$

com $R^{\prime}=\frac{\alpha^{\prime} \phi^{\prime}}{\mu^{\prime}\left(\alpha^{\prime}+\beta^{\prime}\right)}$. Substituindo, então, os valores dos parâmetros, obtemos:

$$
\begin{aligned}
& R^{\prime}=9.950248756 \mathrm{e} \\
& \left(L_{2}, G_{2}\right)=(119.9333333,89.95) .
\end{aligned}
$$

Então, como no caso anterior, segundo o lema 3.1.2, o ponto $\left(L_{1}, G_{1}\right)=(0,0)$ é um ponto de sela e, de acordo com o lema 3.1 .3 , o ponto $\left(L_{2}, G_{2}\right)$ é um foco atrator, já que o valor da expressão $\frac{1}{4 \alpha^{\prime}}\left[\left(\alpha^{\prime}+\beta^{\prime}+\mu^{\prime}\right)^{2}+4 \mu^{\prime}\left(\alpha^{\prime}+\beta^{\prime}\right)\right]$ é

$$
\frac{1}{4 \alpha^{\prime}}\left[\left(\alpha^{\prime}+\beta^{\prime}+\mu^{\prime}\right)^{2}+4 \mu^{\prime}\left(\alpha^{\prime}+\beta^{\prime}\right)\right]=.8120852083
$$

valor menor que $\phi^{\prime}=4$. As figuras $(4.2-\mathrm{a})$ e $(4.2-\mathrm{b})$ representam os retratos de fase do sistema (S-2) para os parâmetros dados em (4.1). Novamente a linha contínua representa o comportamento da solução que, no instante inicial, passa pelo ponto $(L, G)=(5,20)$. 
A seguinte sequência de comandos foi utilizada para os cálculos dessa seção:

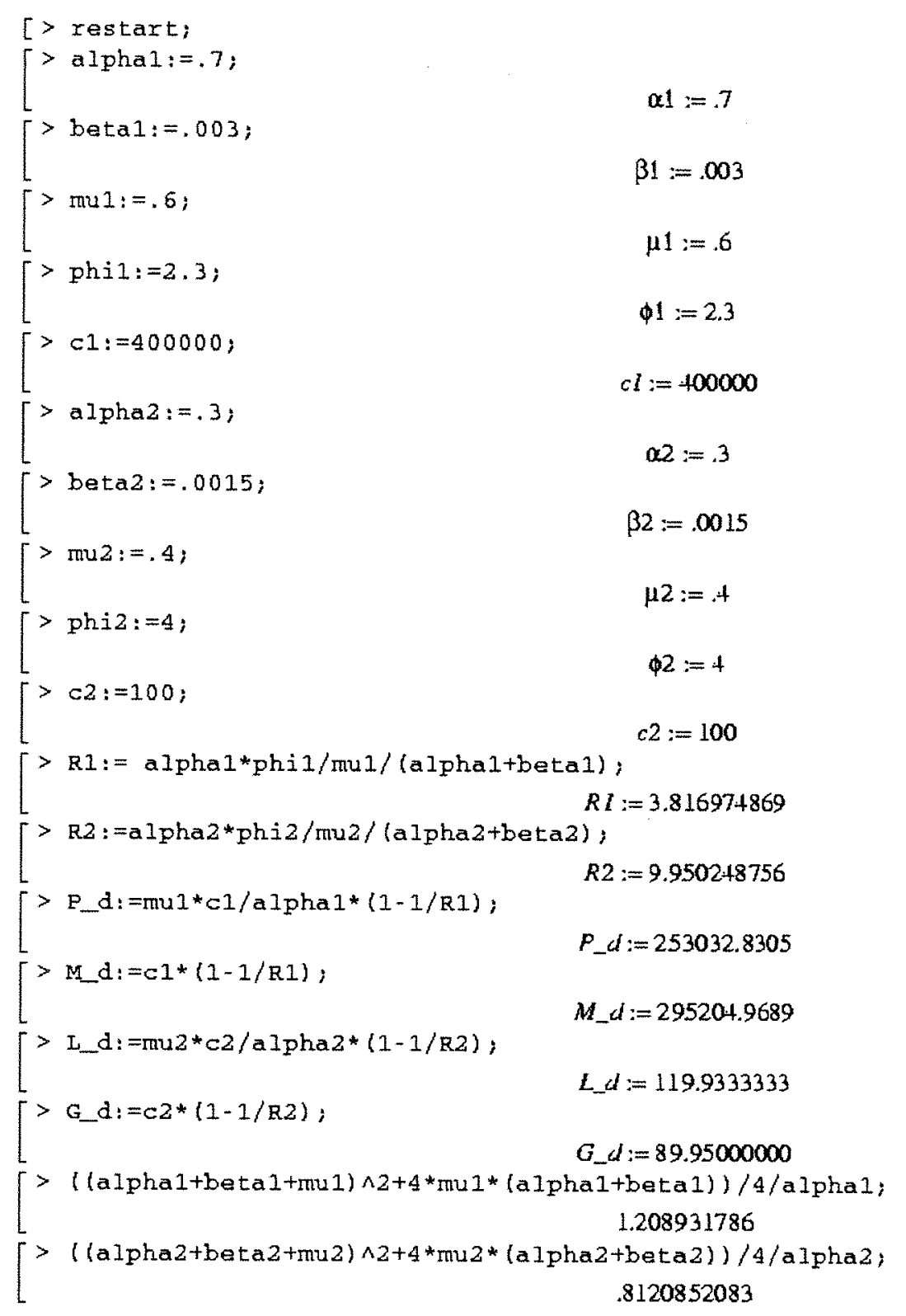

Tabela 4.1: Sequência de comandos do Maple para o sistema (S) desacoplado

Na tabela (4.1), $P_{-} d, M_{-} d, L_{-} d, G_{-} d$, representam as populações, respectivamente, $P$, $M, L, G$, no caso desacoplado $\left(k=k^{\prime}=0\right)$. $R 1$ e $R 2$ são os $R$ e $R^{\prime}$ definidos em (3.9). 


\subsubsection{Cálculo do valor de bifurcação}

De acordo com a seção 3.4, utilizando os valores dos parâmetros dados em (4.1), desejamos encontrar $k$ positivo que satisfaça no ponto $\left(P_{4}, M_{4}, L_{4}, G_{4}\right)$ a equação

$$
\tilde{d}^{2}-\tilde{b} \tilde{c} \tilde{d}+\tilde{e} \tilde{b}^{2}=0
$$

com $\tilde{b}, \tilde{c}, \tilde{d}, \tilde{e}$, dados pelo lema 3.3.11, pág. 74. Isso nos garantirá, pelo lema 3.4.1, pág. 77, que neste ponto de equilíbrio a matriz Jacobiana do sistema (S) apresenta um par de autovalores imaginários puros. Para tanto fixaremos $k^{\prime}=.001$, valor esse sugerido por Ternes [19], e resolveremos numericamente a equação (4.6).

Encontramos, então, quatro valores para $k$, veja tabela (4.2). O valor $k=-.7676843489$. $10^{-4}$ não nos interessa pois procuramos $k$ positivo. $O$ valor $k=.2661534455$ também não nos serve pois este é maior que

$$
k_{\text {max }}=.02201593477 \text {, }
$$

fazendo com que o ponto $\left(P_{4}, M_{4}, L_{4}, G_{4}\right)$ não tenha todas as suas coordenadas positivas, veja lema 3.2.2, pág. 61. Temos então dois valores de $k$ para os quais as populações $P_{4}, M_{4}$, $L_{4}$ e $G_{4}$ são positivas, porém $k=.0001938640812$ é menor que o valor fixado para $k^{\prime}$, que é $k^{\prime}=.001$, o que contradiz o esperado, já que o beneficio $k^{\prime}$ às populações do parasito deveria ser menor que o prejuízo $k$ às populações da praga, uma vez que este último leva a morte da pupa, observação 1.2.2, pág 17.

Portanto, dos quatro valores encontrados para $k$, o único que satisfaz as condições $k \geq k^{\prime}>0$ e $k<k_{\text {max }}$ é

$$
k=.003315398963,
$$

que seguiremos, então, adotando. Note que, como $R>1$ (4.2), $R^{\prime}>1$ (4.4) e $k<k_{\max }$ (4.7), então $\tilde{b}, \tilde{c}, \tilde{d}, \tilde{e}$ são todos positivos, observação 3.3.1, pág 77. Portanto, de acordo com o lema 3.4.1, pág. 77, este valor de $k$, dado em (4.8), deverá fazer com que a matriz Jacobiana do nosso sistema (S) possua um par de autovalores imaginários puros, o que confirmaremos nas seções seguintes. 


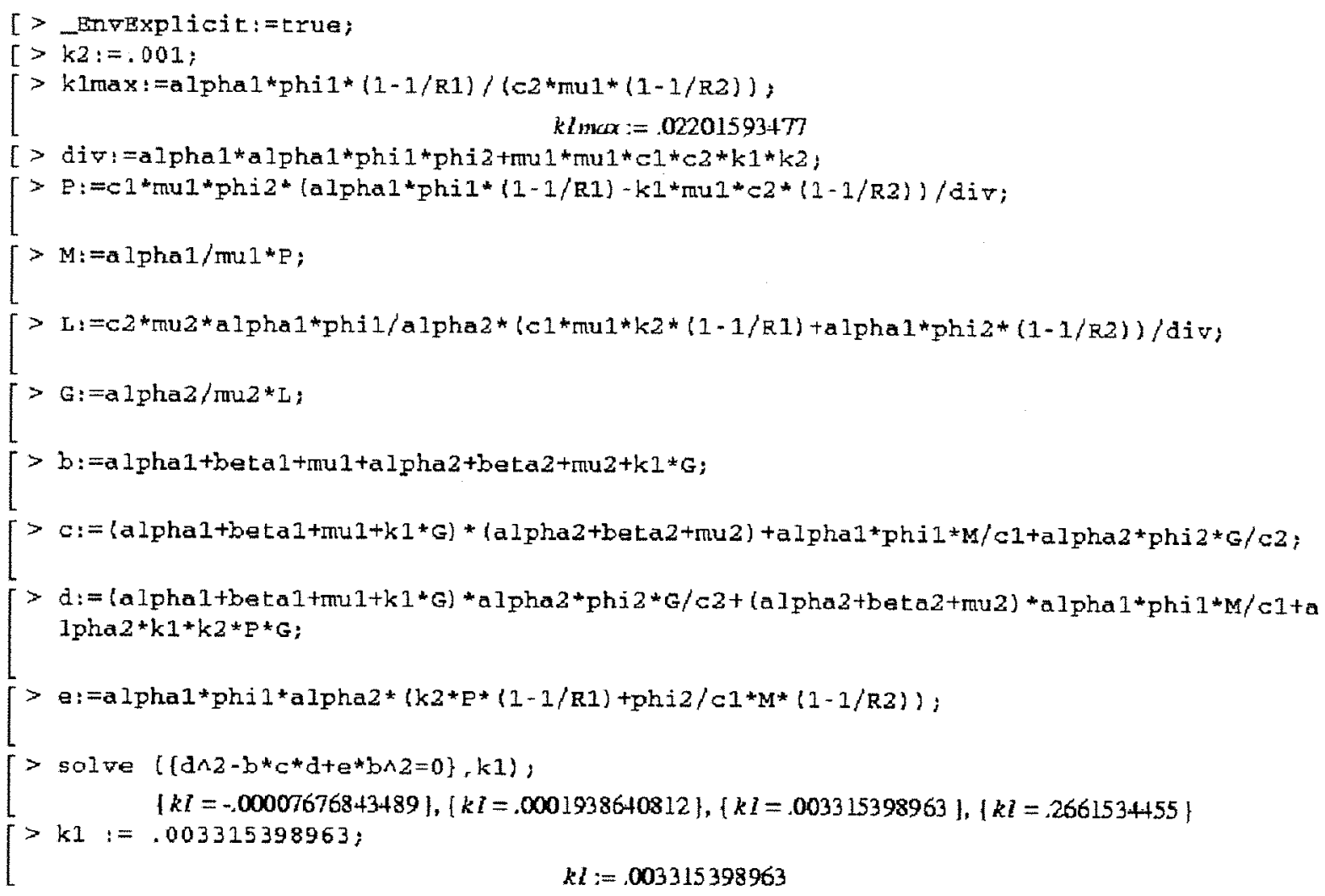

Tabela 4.2: Sequência de comandos do Maple para cálculo do valor de bifurcação

Na tabela (4.2) denotamos o ponto $\left(P_{4}, M_{4}, L_{4}, G_{4}\right)$ simplesmente por $(P, M, L, G)$. Os coeficientes $\tilde{b}, \tilde{c}, \tilde{d}$ e $\tilde{e}$ foram representados apenas por $b, c, d$ e $e$. Por último, $k 1_{\text {max }}$ simboliza a expressão de $k_{\max }$, dada no lema 3.2 .2 .

\subsubsection{Os pontos de equilíbrio e os autovalores}

Como vimos na seção 3.2 o sistema (S) apresenta quatro pontos de equilíbrio, dados por $(3.6-3.9)$, pág. 57.

$$
\left(P_{1}, M_{1}, L_{1}, G_{1}\right)
$$

A matriz Jacobiana $J(x)$ do sistema $(\mathbf{S})$, dada em $(3.13)$, no ponto $\left(P_{1}, M_{1}, L_{1}, G_{1}\right)=$ $(0,0,0,0)$ apresenta quatro autovalores reais (lema 3.3.2, pág. 65). Como $R>1(4.2)$ e $R^{\prime}>1$ (4.4), pelo lema 3.3 .3 , pág. 65 , temos que esse ponto é uma sela $2-2$. 
De fato, calculando numericamente os autovalores encontramos:

$$
\begin{aligned}
& \lambda_{1}=-1.921402457, \quad \lambda_{2}=.6184024569, \\
& \lambda_{3}=.7458016689, \quad \lambda_{4}=-1.447301669 .
\end{aligned}
$$

$$
\left(\mathbf{P}_{2}, \mathbf{M}_{2}, \mathbf{L}_{2}, \mathrm{G}_{2}\right)
$$

A matriz Jacobiana $J(x)$ do sistema (S), dada em (3.13), no ponto $\left(P_{2}, M_{2}, L_{2}, G_{2}\right)$ apresenta quatro autovalores $\lambda_{1}, \lambda_{2}, \lambda_{3}, \lambda_{4}$, dados por (3.15). Como $R>1$ (4.2) os autovalores $\lambda_{3}$ e $\lambda_{4}$ são reais (lema 3.3.5, pág. 67) e como $\phi>\frac{1}{4 \alpha}\left[(\alpha+\beta+\mu)^{2}+4 \mu(\alpha+\beta)\right]$, veja (4.3), $\lambda_{1}$ e $\lambda_{2}$ são complexos conjugados, não reais (lema 3.3.7).

Ainda mais, podemos afirmar que o índice de estabilidade desse ponto de equilíbrio é $\sigma=3$, pois $R>1(4.2)$ e $R^{\prime}>1$ (4.4), veja o lema 3.3.6, pág. 68, implicando que o ponto $\left(P_{2}, M_{2}, L_{2}, G_{2}\right)$ é um hiperbólico 3-1.

Note também que, como $R>1$, nenhuma das populações no equilíbrio $\left(P_{2}, M_{2}, L_{2}, G_{2}\right)$ são negativas, o que biologicamente não faria sentido (observação 3.2.2).

De fato, ao calcularmos numericamente, obtemos

$$
\left(P_{2}, M_{2}, L_{2}, G_{2}\right)=(253032.8305,295204.9689,0,0)
$$

e autovalores dados por

$$
\begin{gathered}
\lambda_{1}=-.6515000000+.8739266270 i, \quad \lambda_{2}=-.6515000000-.8739266270 i, \\
\lambda_{3}=8.430609503, \quad \lambda_{4}=-9.132109503 .
\end{gathered}
$$

$$
\left(\mathbf{P}_{3}, \mathbf{M}_{3}, \mathbf{L}_{3}, \mathbf{G}_{3}\right)
$$

A matriz Jacobiana $J(x)$ do sistema $(\mathbf{S})$, dada em (3.13), no ponto $\left(P_{3}, M_{3}, L_{3}, G_{3}\right)$ também apresenta quatro autovalores $\lambda_{1}, \lambda_{2}, \lambda_{3}, \lambda_{4}$, dados por (3.16). $\lambda_{1}$ e $\lambda_{2}$ são reais, veja demonstração do lema 3.3 .9 , pág. 71 , e $\lambda_{3}$ e $\lambda_{4}$ complexos conjugados, pois

$$
\phi^{\prime}>\frac{1}{4 \alpha^{\prime}}\left[\left(\alpha^{\prime}+\beta^{\prime}+\mu^{\prime}\right)^{2}+4 \mu^{\prime}\left(\alpha^{\prime}+\beta^{\prime}\right)\right]
$$


veja (4.5) e o lema 3.3.10.

Como $R^{\prime}>1(4.4)$ e $k<k_{\max }(4.7)$, podemos afirmar que o indice de estabilidade desse ponto de equilíbrio é $\sigma=3$, veja o lema 3.3.9, implicando que o ponto $\left(P_{3}, M_{3}, L_{3}, G_{3}\right)$ é um hiperbólico 3-1. Note também que, como $R^{\prime}>1$, nenhuma das populações no equilíbrio $\left(P_{3}, M_{3}, L_{3}, G_{3}\right)$ são negativas, o que biologicamente não faria sentido (observação 3.2.2).

De fato, obtemos

$$
\left(P_{3}, M_{3}, L_{3}, G_{3}\right)=(0,0,119.9333333,89.95)
$$

e os autovalores

$$
\begin{gathered}
\lambda_{1}=-2.085228455, \quad \lambda_{2}=.4840083183 \\
\lambda_{3}=-.3507500000+.9779439848 i, \quad \lambda_{4}=-.3507500000-.9779439848 i
\end{gathered}
$$

$$
\left(\mathbf{P}_{4}, \mathbf{M}_{4}, \mathbf{L}_{4}, \mathbf{G}_{4}\right)
$$

Para o valor de $k$, dado em (4.8), esperamos encontrar um par de autovalores imaginários puros. De fato, os valores encontrados para os autovalores foram

$$
\begin{aligned}
& \lambda_{1}=2.846703802 i, \quad \lambda_{2}=-2.846703802 i, \\
& \lambda_{3}=-.2291263418, \quad \lambda_{4}=-3.610577748,
\end{aligned}
$$

para o ponto de equilíbrio

$$
\left(P_{4}, M_{4}, L_{4}, G_{4}\right)=(18543.57758,21634.17385,738.0525862,553.5394397)
$$

Necessitamos, agora, verificar as condições de não-degenerescência e de transversalidade para garantir a existência dessa bifurcação de Hopf. Utilizaremos o procedimento descrito no capítulo 2 , seção 2.2 . 


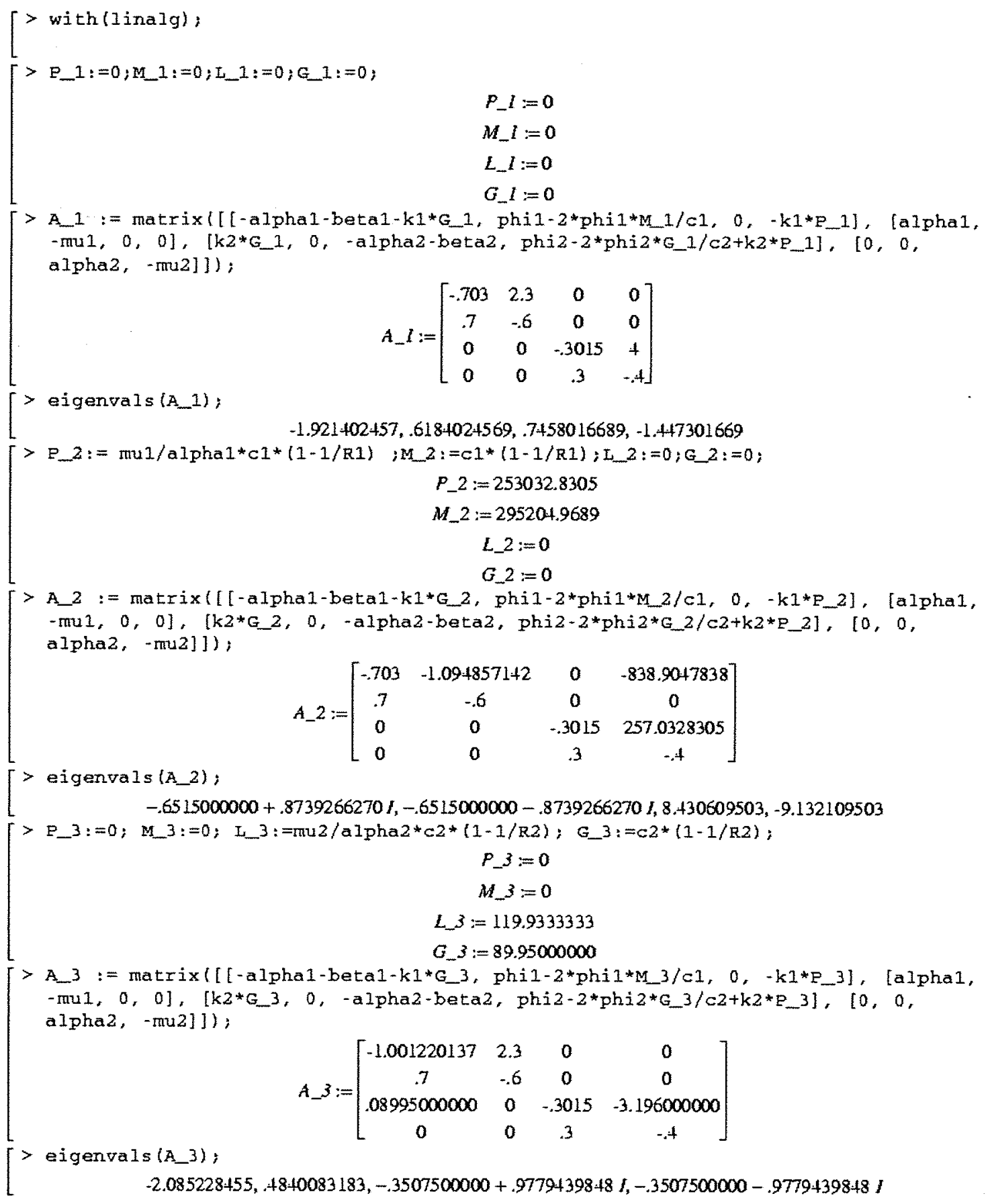

Tabela 4.3: Sequência de comandos do Maple para cálculo dos autovalores nos pontos $\left(P_{1}, M_{1}, L_{1}, G_{1}\right),\left(P_{2}, M_{2}, L_{2}, G_{2}\right)$ e $\left(P_{3}, M_{3}, L_{3}, G_{3}\right)$

O cálculo dos autovalores no ponto $\left(P_{4}, M_{4}, L_{4}, G_{4}\right)$ será mostrado na próxima seção, 
tabela (4.4).

\subsubsection{Cálculo do coeficiente de Lyapunov}

Consideremos, novamente, o sistema

$$
\text { (S) }\left\{\begin{array}{l}
\frac{d P}{d t}=\phi\left(1-\frac{M}{c}\right) M-(\alpha+\beta) P-k P G \\
\frac{d M}{d t}=\alpha P-\mu M \\
\frac{d L}{d t}=\phi^{\prime}\left(1-\frac{G}{c^{\prime}}\right) G-\left(\alpha^{\prime}+\beta^{\prime}\right) L+k^{\prime} P G \\
\frac{d G}{d t}=\alpha^{\prime} L-\mu^{\prime} G
\end{array}\right.
$$

De acordo com o capítulo 2, escreveremos tal sistema na forma (2.28), veja pág. 39,

$$
\left(\begin{array}{c}
\frac{d P}{d t} \\
\frac{d M}{d t} \\
\frac{d L}{d t} \\
\frac{d G}{d t}
\end{array}\right)=\left(\begin{array}{cccc}
-(\alpha+\beta) & \phi & 0 & 0 \\
\alpha & -\mu & 0 & 0 \\
0 & 0 & -\left(\alpha^{\prime}+\beta^{\prime}\right) & \phi^{\prime} \\
0 & 0 & \alpha^{\prime} & -\mu^{\prime}
\end{array}\right)\left(\begin{array}{c}
P \\
M \\
L \\
G
\end{array}\right)+\left(\begin{array}{c}
-\phi \frac{M^{2}}{c}-k P G \\
0 \\
-\phi^{\prime} \frac{G^{2}}{c^{\prime}}+k^{\prime} P G \\
0
\end{array}\right)
$$

Para analisar o ponto $\left(P_{4}, M_{4}, L_{4}, G_{4}\right)$, fazemos

$$
\left\{\begin{array}{l}
\tilde{P}=P-P_{4} \\
\tilde{M}=M-M_{4} \\
\tilde{L}=L-L_{4} \\
\tilde{G}=G-G_{4}
\end{array}\right.
$$

trasladando esse sistema para analisá-lo na origem, obtendo:

$$
\left(\begin{array}{c}
\frac{d \tilde{P}}{d t} \\
\frac{d \tilde{M}}{d t} \\
\frac{d \tilde{L}}{d t} \\
\frac{d \tilde{G}}{d t}
\end{array}\right)=\left(\begin{array}{c}
\phi M_{4}\left(1-\frac{M_{4}}{c}\right)-(\alpha+\beta) P_{4}-k P_{4} G_{4} \\
\alpha P_{4}-\mu M_{4} \\
\phi^{\prime} G_{4}\left(1-\frac{G_{4}}{c^{\prime}}\right)-\left(\alpha^{\prime}+\beta^{\prime}\right) L_{4}+k^{\prime} P_{4} G_{4} \\
\alpha^{\prime} L_{4}-\mu^{\prime} G_{4}
\end{array}\right)+
$$




$$
\begin{aligned}
& +\left(\begin{array}{cccc}
-(\alpha+\beta)-k G_{4} & \phi\left(1-2 \frac{M_{4}}{c}\right) & 0 & -k P_{4} \\
\alpha & -\mu & 0 & 0 \\
k^{\prime} G_{4} & 0 & -\left(\alpha^{\prime}+\beta^{\prime}\right) & \phi^{\prime}\left(1-2 \frac{G_{4}}{c^{\prime}}\right)+k^{\prime} P_{4} \\
0 & 0 & \alpha^{\prime} & -\mu^{\prime}
\end{array}\right)\left(\begin{array}{c}
\tilde{P} \\
\tilde{M} \\
\tilde{L} \\
\tilde{G}
\end{array}\right)+ \\
& +\left(\begin{array}{c}
-\phi^{\frac{\tilde{M}^{2}}{c}}-k \tilde{P} \tilde{G} \\
0 \\
-\phi^{\prime} \frac{\tilde{G}^{2}}{c^{\prime}}+k^{\prime} \tilde{P} \tilde{G} \\
0
\end{array}\right),
\end{aligned}
$$

onde $\left(P_{4}, M_{4}, L_{4}, G_{4}\right)$ é dado em (3.9), pág. 58. Chamaremos esse sistema de (S4). Por questão de praticidade tornaremos a tratar $\tilde{P}, \tilde{M}, \tilde{L}, \tilde{G}$, por somente, $P, M, L$ e $G$.

Note que no sistema (S4), os termos constantes

$$
\left(\begin{array}{c}
\phi M_{4}\left(1-\frac{M_{4}}{c}\right)-(\alpha+\beta) P_{4}-k P_{4} G_{4} \\
\alpha P_{4}-\mu M_{4} \\
\phi^{\prime} G_{4}\left(1-\frac{G_{4}}{c^{\prime}}\right)-\left(\alpha^{\prime}+\beta^{\prime}\right) L_{4}+k^{\prime} P_{4} G_{4} \\
\alpha^{\prime} L_{4}-\mu^{\prime} G_{4}
\end{array}\right)
$$

são nulos, já que $\left(P_{4}, M_{4}, L_{4}, G_{4}\right)$ é ponto de equilíbrio de (S) e, portanto, satisfaz as quatro equações

$$
\left\{\begin{array}{l}
\frac{d P}{d t}=\phi\left(1-\frac{M}{c}\right) M-(\alpha+\beta) P-k P G=0 \\
\frac{d M}{d t}=\alpha P-\mu M=0 \\
\frac{d L}{d t}=\phi^{\prime}\left(1-\frac{G}{c^{\prime}}\right) G-\left(\alpha^{\prime}+\beta^{\prime}\right) L+k^{\prime} P G=0 \\
\frac{d G}{d t}=\alpha^{\prime} L-\mu^{\prime} G=0
\end{array} .\right.
$$

Assim sendo, temos o sistema (S4) dado por

$$
\left(\begin{array}{c}
\frac{d P}{d t} \\
\frac{d M}{d t} \\
\frac{L}{d t} \\
\frac{d G}{d t}
\end{array}\right)=\left(\begin{array}{cccc}
-(\alpha+\beta)-k G_{4} & \phi\left(1-2 \frac{M_{4}}{c}\right) & 0 & -k P_{4} \\
\alpha & -\mu & 0 & 0 \\
k^{\prime} G_{4} & 0 & -\left(\alpha^{\prime}+\beta^{\prime}\right) & \phi^{\prime}\left(1-2 \frac{G_{4}}{c^{\prime}}\right)+k^{\prime} P_{4} \\
0 & 0 & \alpha^{\prime} & -\mu^{\prime}
\end{array}\right)\left(\begin{array}{c}
P \\
M \\
L \\
G
\end{array}\right)
$$




$$
+\left(\begin{array}{c}
-\phi \frac{M^{2}}{c}-k P G \\
0 \\
-\phi^{\prime} \frac{G^{2}}{c^{\prime}}+k^{\prime} P G \\
0
\end{array}\right)
$$

Chamaremos de

$$
F(x)=\left(\begin{array}{c}
-\phi^{\frac{M^{2}}{c}}-k P G \\
0 \\
-\phi^{\prime} \frac{G^{2}}{c^{\prime}}+k^{\prime} P G \\
0
\end{array}\right)
$$

a função que corresponde a parte não linear e de

$$
A=\left(\begin{array}{cccc}
-(\alpha+\beta)-k G_{4} & \phi\left(1-2 \frac{M_{4}}{c}\right) & 0 & -k P_{4} \\
\alpha & -\mu & 0 & 0 \\
k^{\prime} G_{4} & 0 & -\left(\alpha^{\prime}+\beta^{\prime}\right) & \phi^{\prime}\left(1-2 \frac{G_{4}}{c^{\prime}}\right)+k^{\prime} P_{4} \\
0 & 0 & \alpha^{\prime} & -\mu^{\prime}
\end{array}\right)
$$

a matriz correspondente a parte linear de (S4). Portanto, se $x=(P, M, L, G)$, temos o sistema na forma

$$
\dot{x}=A x+F(x)
$$

como queríamos, veja capítulo 2 , seção 2.2. Note que a matriz $A$ é exatamente a matriz $J(x)$, matriz Jacobiana do sistema (S), aplicada no ponto $\left(P_{4}, M_{4}, L_{4}, G_{4}\right)$, como havia de ser.

Tomando $k=k_{0}=.003315398963$ como dado em (4.8), encontramos os autovalores

$$
\begin{aligned}
& \lambda_{1}=2.846703802 i \\
& \lambda_{2}=-2.846703802 i \\
& \lambda_{3}=-.2291263418, \\
& \lambda_{4}=-3.610577748,
\end{aligned}
$$

como vimos na subseção 4.1.3. Conforme a seção 2.2, pág. 38, chamaremos de $q$ o autovetor correspondente a $\lambda_{1}=i \omega_{0} \mathrm{e}$, portanto, $\omega_{0}=2.846703802$. Calculando o autovetor 
correspondente a $\lambda_{1}$ obtemos

$$
q=\left(\begin{array}{c}
820.5542609+1080.774610 i \\
295.1756045-139.5588184 i \\
862.8021803+130.4940530 i \\
26.01486634-87.27100717 i
\end{array}\right)
$$

$\mathrm{Na}$ verdade sabemos que qualquer múltiplo de $q$ será autovetor correspondente a $\lambda_{1}=i \omega_{0}$, o comprimento escolhido, porém, alterará o valor do coeficiente de Lyapunov, mas não o seu sinal, observação 2.1.1, pág.37. Mais adiante, na subseção 4.1.7, daremos maiores explicações dessa escolha. Por hora adotamos este valor para $q$ e calculamos agora $p$, o autovetor de $A^{t}$ correspondente ao autovalor $-i \omega_{0}$ e, normalizando $p$ para que $\langle p, q\rangle=1$, obtemos

$$
p=\left(\begin{array}{c}
.00003314748646+.00006274424412 i \\
-.00003846764141+.00003199241887 i \\
.0005233172006+.00007678211168 i \\
.001254520214-.004888597529 i
\end{array}\right)
$$

Lema 4.1.1 As funções multilineares $B(x, y)$ e $C(x, y, z), x, y, z \in \mathbb{R}^{4}$, para o sistema (S4) ficam dadas por:

$$
B(x, y)=\left(\begin{array}{c}
-\frac{2 \phi}{c} x_{2} y_{2}-k x_{1} y_{4}-k x_{4} y_{1} \\
0 \\
-\frac{2 \phi^{\prime}}{c^{\prime}} x_{4} y_{4}+k^{\prime} x_{1} y_{4}+k^{\prime} x_{4} y_{1} \\
0
\end{array}\right)
$$

$e$

$$
C(x, y, z) \equiv 0
$$

Demonstração 4.1.1 Notemos primeiramente que as funções $B(x, y)$ e $C(x, y, z)$, como acabamos de definir, satisfazem a fórmula (2.36): 
Seja $x=(P, M, L, G)$, então,

$$
B(x, x)=\left(\begin{array}{c}
-\frac{2 \phi}{c} M^{2}-2 k P G \\
0 \\
-\frac{2 \phi^{\prime}}{c^{\prime}} G^{2}+2 k^{\prime} P G \\
0
\end{array}\right)
$$

$e$

$$
C(x, x, x) \equiv 0
$$

Portanto, $F(x)=\frac{1}{2} B(x, x)+\frac{1}{6} C(x, x, x)+O\left(\|x\|^{4}\right)$.

Para encontrarmos as funções $B(x, y)$ e $C(x, y, z)$ utilizamos as fórmulas:

$$
B_{i}(x, y)=\left.\sum_{j, k=1}^{2} \frac{\partial^{2} F_{i}(\eta, 0)}{\partial \eta_{j} \partial \eta_{k}}\right|_{\eta=0} x_{j} y_{k}, \quad i=1,2,3,4
$$

$e$

$$
C_{i}(x, y, z)=\left.\sum_{j, k, l=1}^{2} \frac{\partial^{3} F_{i}(\eta, 0)}{\partial \eta_{j} \partial \eta_{k} \partial \eta_{l}}\right|_{\eta=0} x_{j} y_{k} z_{l}, \quad i=1,2,3,4
$$

vistas no capítulo 2.

Para calcular $B_{1}(x, y)$, por exemplo, consideramos $F_{1}(P, M, L, G)=-\phi \frac{M^{2}}{c}-k P G e$ calculamos:

$$
\begin{array}{cccc}
\frac{\partial F_{1}}{\partial P}=-k G & \frac{\partial F_{1}}{\partial M}=-2 \frac{\phi}{c} M & \frac{\partial F_{1}}{\partial L}=0 & \frac{\partial F_{1}}{\partial G}=-k P \\
\frac{\partial}{\partial P}\left(\frac{\partial F_{1}}{\partial P}\right)=0 & \frac{\partial}{\partial P}\left(\frac{\partial F_{1}}{\partial M}\right)=0 & \frac{\partial}{\partial P}\left(\frac{\partial F_{1}}{\partial L}\right)=0 & \frac{\partial}{\partial P}\left(\frac{\partial F_{1}}{\partial G}\right)=-k \\
\frac{\partial}{\partial M}\left(\frac{\partial F_{1}}{\partial P}\right)=0 & \frac{\partial}{\partial M}\left(\frac{\partial F_{1}}{\partial M}\right)=-2 \frac{\phi}{c} & \frac{\partial}{\partial M}\left(\frac{\partial F_{1}}{\partial L}\right)=0 & \frac{\partial}{\partial M}\left(\frac{\partial F_{1}}{\partial G}\right)=0 \\
\frac{\partial}{\partial L}\left(\frac{\partial F_{1}}{\partial P}\right)=0 & \frac{\partial}{\partial L}\left(\frac{\partial F_{1}}{\partial M}\right)=0 & \frac{\partial}{\partial L}\left(\frac{\partial F_{1}}{\partial L}\right)=0 & \frac{\partial}{\partial L}\left(\frac{\partial F_{1}}{\partial G}\right)=0 \\
\frac{\partial}{\partial G}\left(\frac{\partial F_{1}}{\partial P}\right)=-k & \frac{\partial}{\partial G}\left(\frac{\partial F_{1}}{\partial M}\right)=0 & \frac{\partial}{\partial G}\left(\frac{\partial F_{1}}{\partial L}\right)=0 & \frac{\partial}{\partial G}\left(\frac{\partial F_{1}}{\partial G}\right)=0
\end{array}
$$

Portanto,

$$
\begin{aligned}
& B_{1}(x, y)=0 x_{1} y_{1} \quad+0 x_{1} y_{2} \quad+0 x_{1} y_{3}-k x_{1} y_{4} \\
& +0 x_{2} y_{1} \quad-2 \frac{\phi}{c} x_{2} y_{2} \quad+0 x_{2} y_{3} \quad+0 x_{2} y_{4} \\
& +0 x_{3} y_{1}+0 x_{3} y_{2}+0 x_{3} y_{3}+0 x_{3} y_{4} \\
& -k x_{4} y_{1}+0 x_{4} y_{2}+0 x_{4} y_{3}+0 x_{4} y_{4} \text {. }
\end{aligned}
$$


Cálculos análogos para as funções $B_{2}(x, y), B_{3}(x, y), B_{4}(x, y), C_{1}(x, y, z), C_{2}(x, y, z)$, $C_{3}(x, y, z)$ e $C_{4}(x, y, z)$ completam o resultado.

Assim $B(q, q)$ e $B(q, \bar{q})$ ficam dados por:

$$
\begin{gathered}
B(q, q)=\left(\begin{array}{c}
-767.7418261+289.3499796 i \\
0 \\
786.4902302+276.2661945 i \\
0
\end{array}\right), \\
B(q, \bar{q})=\left(\begin{array}{c}
482.6477605 \\
0 \\
-809.3875158+.6 \cdot 10^{-7} i \\
0
\end{array}\right) .
\end{gathered}
$$

Seja $s=A^{-1} B(q, \bar{q})$ e $r=\left(2 i \omega_{0} I-A\right)^{-1} B(q, q)$. Então $s$ e $r$ ficam dados por:

$$
\begin{gathered}
s=\left(\begin{array}{r}
-1622.977370+.9904140546 \cdot 10^{-7} i \\
-1893.473598+.1155483063 \cdot 10^{-6} i \\
-5.359116331-.3117318364 \cdot 10^{-9} i \\
-4.019337253-.2337988771 \cdot 10^{-9} i
\end{array}\right), \\
r=\left(\begin{array}{c}
71.87520338+68.12253398 i \\
9.204672581-7.866965075 i \\
83.57142169-174.4554220 i \\
-8.839482676-5.024621730 i
\end{array}\right)
\end{gathered}
$$


Calculando, então, $B(q, s)$ e $B(\bar{q}, r)$, temos:

$$
\begin{gathered}
B(q, s)=\left(\begin{array}{c}
157.3431460-458.2261622 i \\
0 \\
-37.15462194+109.2331432 i \\
0
\end{array}\right) \\
B(\bar{q}, r)=\left(\begin{array}{c}
55.51908921-44.66417557 i \\
0 \\
-33.44272420+85.64696716 i \\
0
\end{array}\right)
\end{gathered}
$$

Portanto, $<p, B(q, s)>\mathrm{e}<p, B(\bar{q}, r)>$, valem:

$$
\begin{aligned}
& <p, B(q, s)>=-.03459202572+.03495497077 i \\
& <p, B(\bar{q}, r)>=-.01188709949+.04242432564 i
\end{aligned}
$$

Como

$$
C(q, q, \bar{q}) \equiv 0, \quad<p, B\left(q, A^{-1} B(q, \bar{q})\right)>=<p, B(q, s)>
$$

e

$$
<p, B\left(\bar{q},\left(2 i \omega_{0} I-A\right)^{-1} B(q, q)\right)>=<p, B(\bar{q}, r)>,
$$

a fórmula para cálculo do coeficiente de Lyapunov, vista em (2.26), pág. 37,

$$
\begin{aligned}
l_{1}(0)= & \frac{1}{2 \omega_{0}} \operatorname{Re}\left[<p, C(q, q, \bar{q})>-2<p, B\left(q, A^{-1} B(q, \bar{q})\right)>\right. \\
& \left.+<p, B\left(\bar{q},\left(2 i \omega_{0} I-A\right)^{-1} B(q, q)\right)>\right],
\end{aligned}
$$

fica agora dada por

$$
l_{1}(0)=\frac{1}{2 \omega_{0}} \operatorname{Re}[-2<p, B(q, s)>+<p, B(\bar{q}, r)>]
$$

Assim, temos que

$$
l_{1}\left(k_{0}\right)=.003535224196
$$

e, portanto, que a bifurcação de Hopf é não-degenerada pois $l_{1} \neq 0$. 


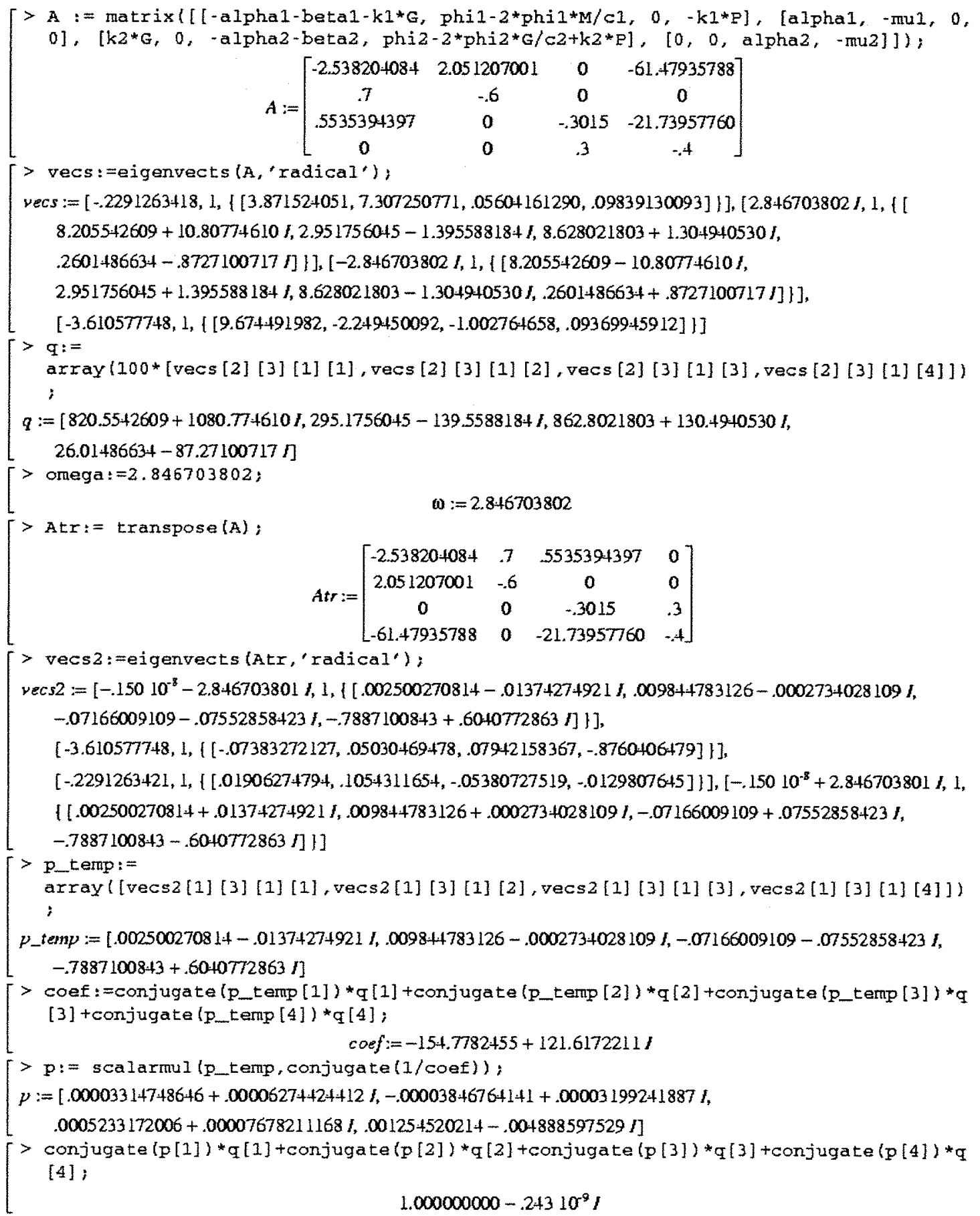

Tabela 4.4: Sequência de comandos do Maple para cálculo dos autovetores $p$ e $q$

Aqui na tabela (4.4) $A^{t}$ foi representada por Atr e o último comando só foi implementado para conferirmos que $\langle p, q\rangle=1$. 


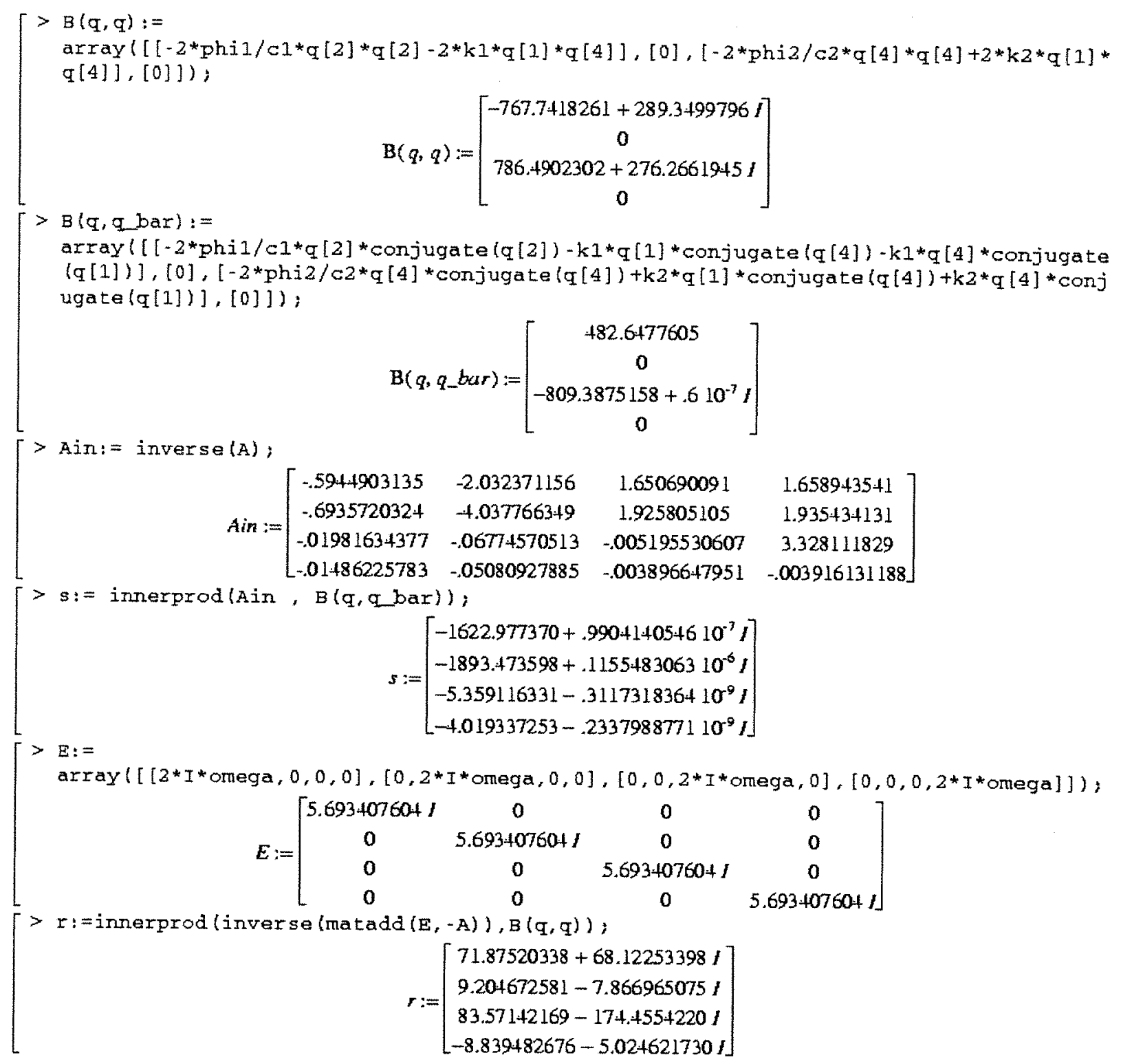

Tabela 4.5: Primeira sequência de comandos do Maple para cálculo do coeficiente de Lyapunov

Nas tabelas (4.5) e (4.6), Ain representa a inversa de $A\left(A^{-1}\right), q_{-}$bar o vetor $\bar{q}$ e $E$ representa a expressão $2 i \omega_{0} I$. 


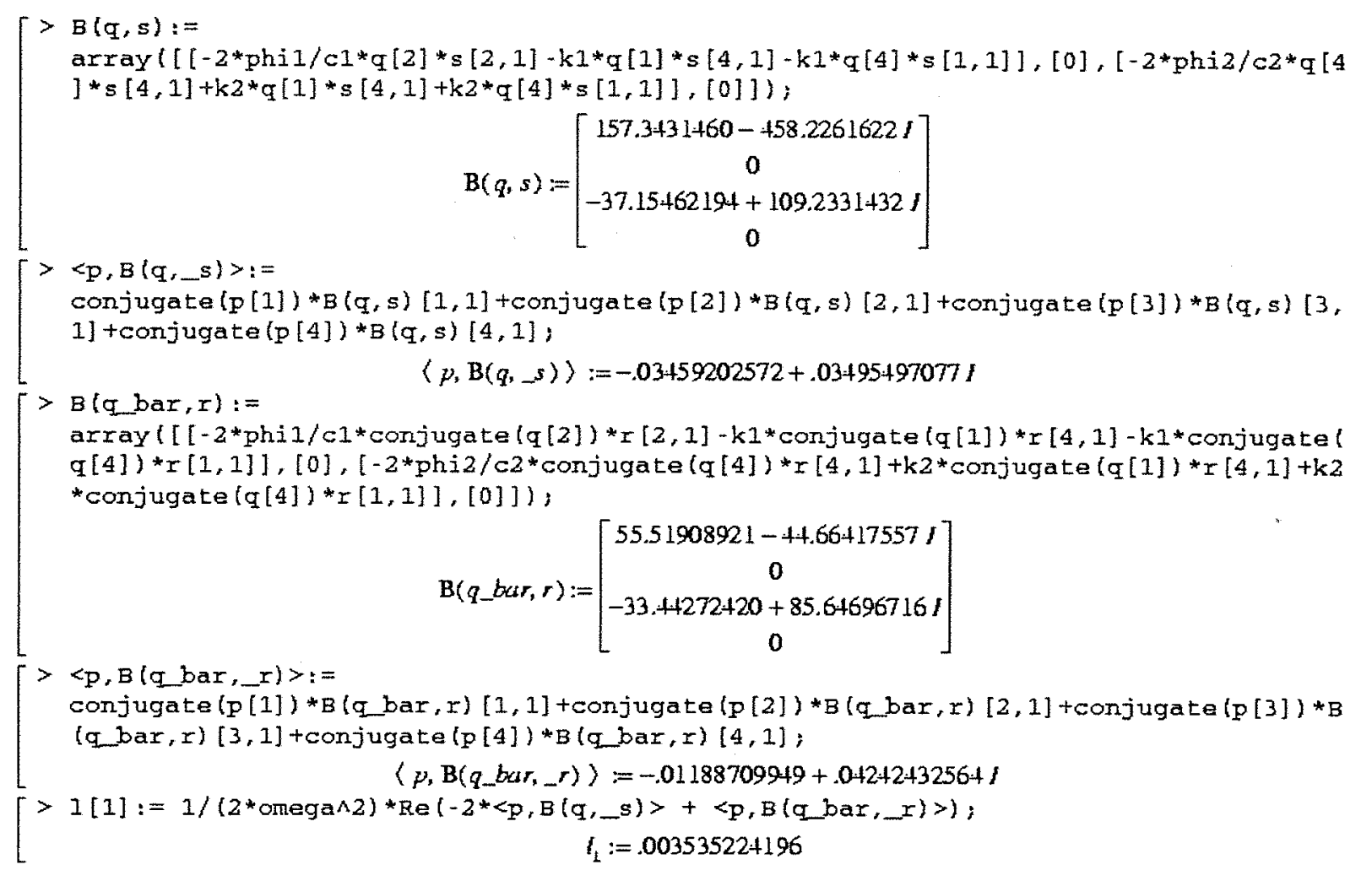

Tabela 4.6: Segunda sequência de comandos do Maple para cálculo do coeficiente de Lyapunov

\subsubsection{A condição de transversalidade}

Consideremos a matriz $A$ do sistema (S4), dada em (4.9), mantendo a dependência com relação ao parâmetro $k$, conforme sugere o lema 2.2 .5 , pág. 48. Calculamos, então, $\frac{d A}{d k}=$ $A^{\prime}(k)$, obtendo:

$$
A^{\prime}(k)=\left(\begin{array}{cccc}
-G_{4}^{\prime}-k \frac{d G_{4}}{d k} & -2 \frac{\phi}{c} \frac{d M_{4}}{d k} & 0 & -P_{4}-k \frac{d P_{4}}{d k} \\
0 & 0 & 0 & 0 \\
k^{\prime} \frac{d G_{4}}{d k} & 0 & 0 & -2 \frac{\phi^{\prime}}{c^{\prime}} \frac{d G_{4}}{d k}+k^{\prime} \frac{d P_{4}}{d k} \\
0 & 0 & 0 & 0
\end{array}\right) .
$$

Calculando, agora, $\gamma^{\prime}\left(k_{0}\right)=R e<p, A^{\prime}\left(k_{0}\right) q>$, obtemos

$$
\gamma^{\prime}\left(k_{0}\right)=-48.2221612
$$

$\mathrm{e}$, portanto, $\gamma^{\prime}\left(k_{0}\right) \neq 0$ como queríamos. 


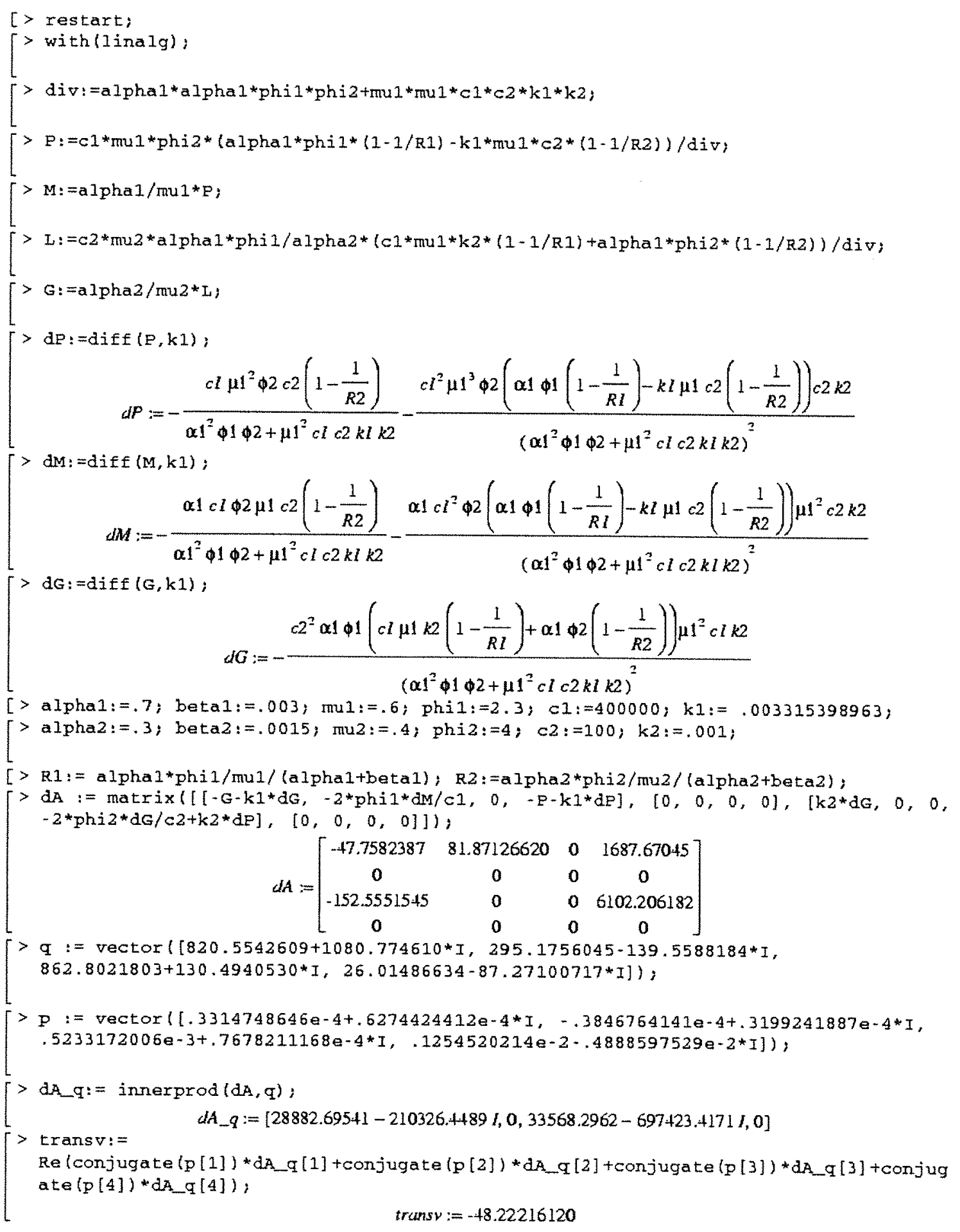

Tabela 4.7: Sequência de comandos do Maple para o cálculo de $\gamma^{\prime}\left(k_{0}\right)$

Na tabela (4.7), $d A$ representa a matriz $A^{\prime}\left(k_{0}\right), d A \_q$ o produto $A^{\prime}\left(k_{0}\right) q$ e transv é $\gamma^{\prime}\left(k_{0}\right)$. 


\subsubsection{Simulação numérica de diversas condições de campo}

Satisfeitas as duas condições de Hopf $\left(l_{1}\left(k_{0}\right)>0\right.$ e $\left.\gamma^{\prime}\left(k_{0}\right)<0\right)$ garantimos o surgimento de um ciclo periódico na dinâmica das populações de ambas as espécies. Faremos, então, algumas simulações do comportamento das populações da praga e do parasito em torno do valor crítico $k_{0}=.003315398963$, calculado em (4.8).

caso $\left(\mathrm{k}>\mathrm{k}_{0}\right)$

Inicialmente adotaremos um valor para $k$ maior que $k_{0}$ (e obviamente maior que $k^{\prime} \mathrm{e}$ menor que $k_{\text {max }}$ ) e procuraremos analisar o comportamento das soluções do sistema (S) em torno do quarto ponto de equilíbrio. Seja, portanto,

$$
k=.005 \text {. }
$$

Para este valor de $k$, o ponto $x_{4}=\left(P_{4}, M_{4}, L_{4}, G_{4}\right)$ fica dado por

$$
\left(P_{4}, M_{4}, L_{4}, G_{4}\right)=(11523.18712,13443.71831,504.0395708,378.0296781)
$$

e os autovalores neste ponto dados por

$$
\begin{array}{ll}
\lambda_{1}=-.06765502811+2.360058484 i, & \lambda_{2}=-.06765502811-2.360058484 i, \\
\lambda_{3}=-3.548748258, & \lambda_{4}=-.2105900771 .
\end{array}
$$

Note que os dois autovalores complexos conjugados têm parte real negativa, implicando que o ponto de equilíbrio é um atrator. Isso significa que devemos ter um ciclo limite numa vizinhança deste ponto, uma vez que o coeficiente de Lyapunov é positivo. Este é um ciclo cuja variedade estável tem dimensão 3 e a variedade instável dimensão 2.

A figura (4.3) mostra o comportamento das populações adultas da praga, $M$, e do parasito, $G$, com valores iniciais próximos ao equilíbrio, dados por

$$
\left(P_{0}, M_{0}, L_{0}, G_{0}\right)=(11000,13000,500,400)
$$

após dez meses ( $n=300$, onde $n$ é o número de dias). Devido à proximidade do equilíbrio a solução permanece no interior da bacia de atração do ponto $x_{4} \mathrm{e}$, portanto, tende a esse 
equilíbrio. Entende-se por bacia de atração o lugar geométrico dos pontos cujas órbitas que por eles passam tendem ao ponto $x_{4}$ quando o tempo cresce, isto é,

$$
\left\{x \in \mathbb{R}^{4}: \psi^{t} x \rightarrow x_{4}, \text { parat } \rightarrow+\infty\right\}
$$

onde $\psi^{t}$ denota o fluxo associado ao sistema (S).

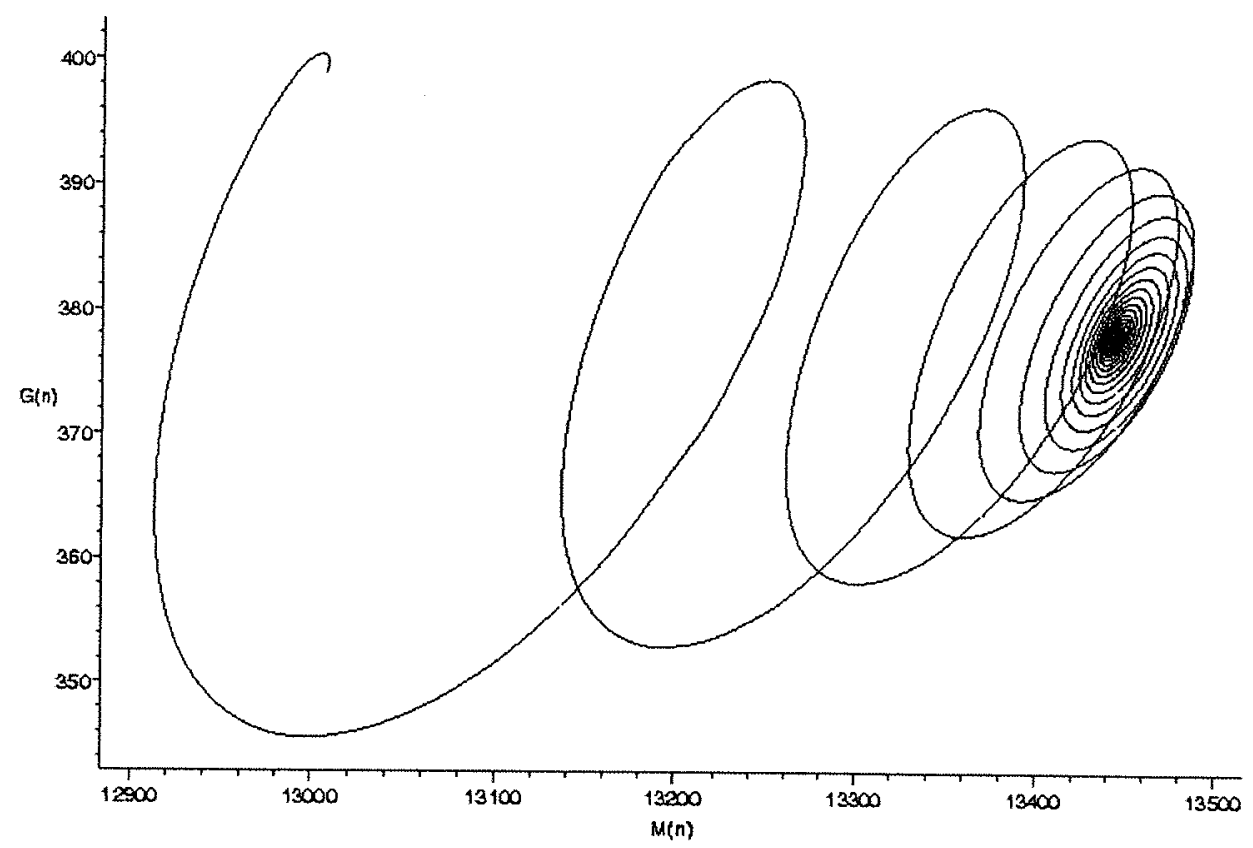

Figura 4.3: $M \times G \mid$ próximo ao equilíbrio $\mid n=300$

A figura (4.4) mostra o comportamento das populações da pupa da praga, $P$, e da larva do parasito, $L$, para os mesmos valores iniciais e mesmo espaço de tempo, evidenciando comportamento semelhante.

As figuras (4.5) e (4.6) mostram o comportamento das populações $M$ e $G$ e das populações $P$ e $L$, com dado inicial dado por

$$
\left(P_{0}, M_{0}, L_{0}, G_{0}\right)=(11000,13000,100,100)
$$

portanto mais afastado do equilíbrio, após 23 dias. Neste caso a solução que parte de valores iniciais distantes do equilíbrio está fora da bacia de atração do equilíbrio e, portanto, rapidamente (três semanas) se afasta desse equilíbrio. 


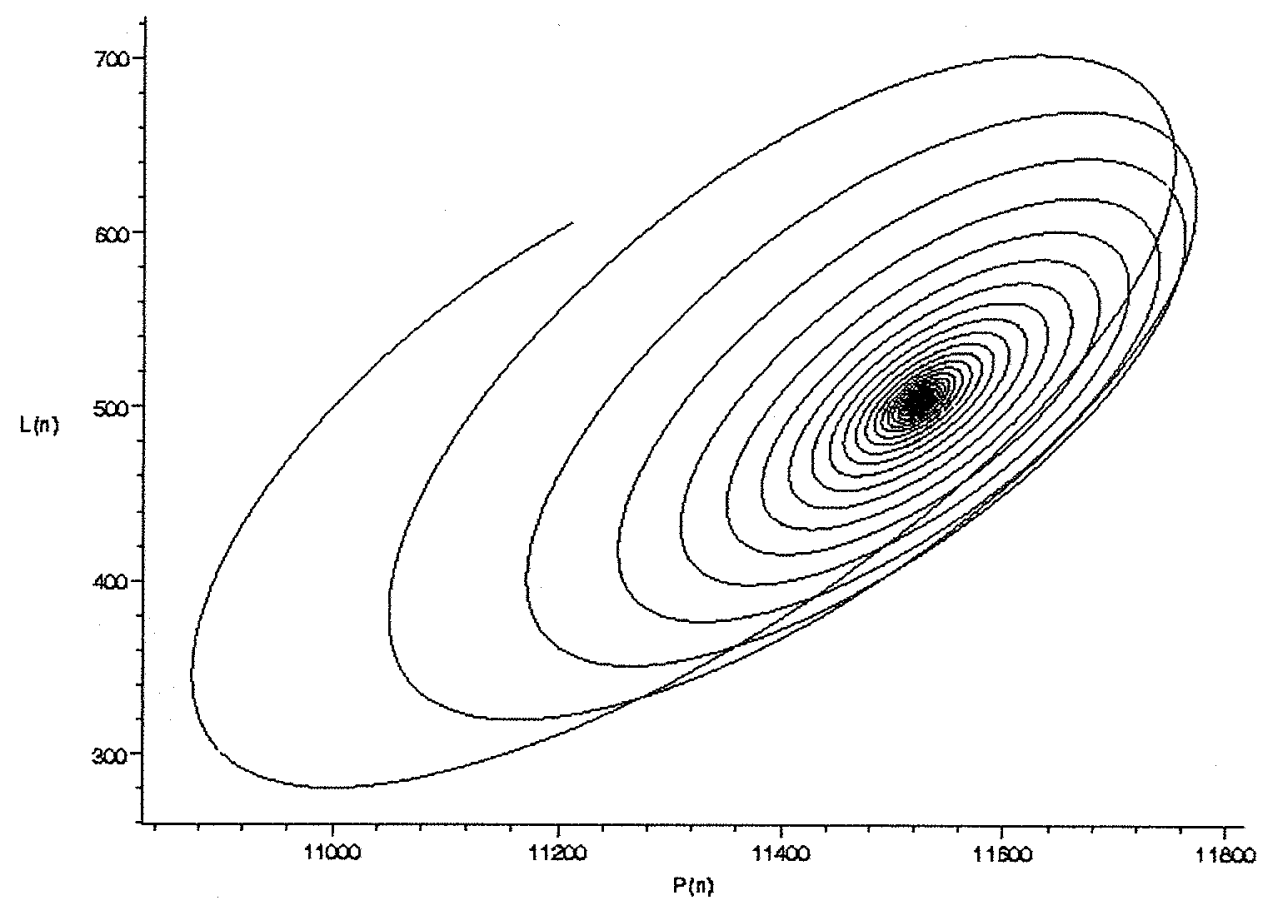

Figura 4.4: $P \times L \mid$ próximo ao equilíbrio $\mid n=300$

A tabela (4.8), pág. 113, mostra os comandos do Maple usados para gerar os gráficos apresentados. O equilíbrio foi representado por $(P 4, M 4, L 4, G 4)$ e o parâmetro $n$ representa o tempo, que neste caso é dado em dias. Para os próximos gráficos omitiremos a sequência de comandos, uma vez que foram gerados do mesmo modo, simplesmente alterando os valores iniciais e o tempo $n$. 


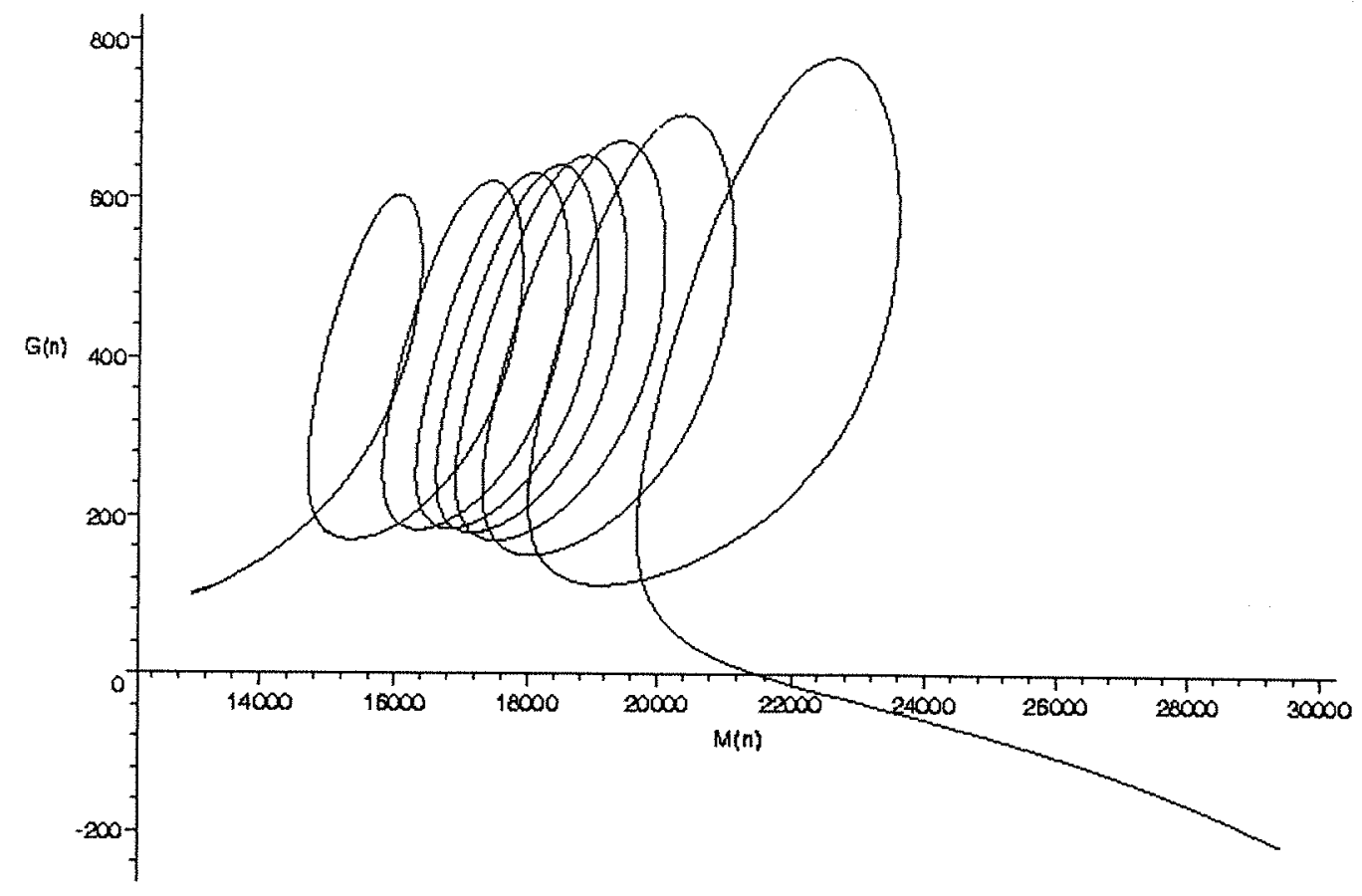

Figura 4.5: $M \times G \mid$ distante do equilíbrio $\mid n=23$

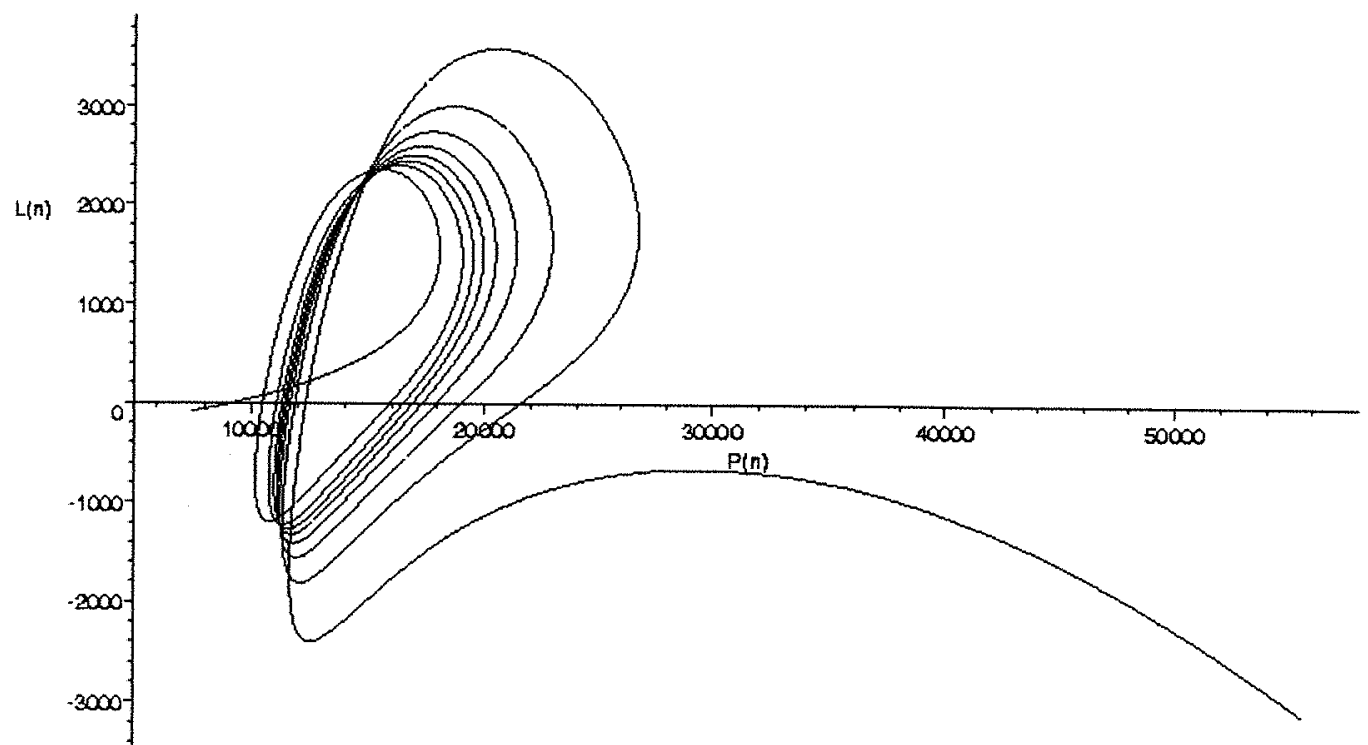

Figura 4.6: $P \times L \mid$ distante do equilíbrio $\mid n=23$ 
caso $\left(\mathrm{k}<\mathrm{k}_{0}\right)$

Adotando, agora,

$$
k=.001,
$$

valor este menor que o valor crítico $k_{0}$ (e igual a $\left.k^{\prime}\right)$, já podemos prever que os autovalores complexos conjugados deverão possuir parte real positiva e que nenhum ciclo limite surgirá na vizinhança do equilíbrio. De fato, para este valor de $k$, o quarto ponto de equilíbrio fica dado por

$$
\left(P_{4}, M_{4}, L_{4}, G_{4}\right)=(57587.30696,67185.19147,2039.510233,1529.632675)
$$

e os autovalores neste ponto dados por

$$
\begin{array}{ll}
\lambda_{1}=.1405403430+4.545209274 i, & \lambda_{2}=.1405403430-4.545209274 i, \\
\lambda_{3}=-3.529998452, & \lambda_{4}=-.2852149089 .
\end{array}
$$

Vamos analisar os dois casos $\left(k>k_{0}\right.$ e $\left.k<k_{0}\right)$ em três situações de campo, as quais designaremos por:

(i) baixa infestação: $P_{0}=1000, M_{0}=1000$;

(ii) média infestação: $P_{0}=5000, M_{0}=5000$;

(iii) alta infestação: $P_{0}=10000, M_{0}=10000$.

$$
\underline{k=.001}
$$

A figura (4.7) mostra o comportamento das populações $M$ e $G$ na situação (i) com a inserção de dez adultos do parasito, $L_{0}=0$ e $G_{0}=10$. A solução rapidamente direciona-se à variedade bidimensional instável e passa a oscilar até que, em pouco mais de um mês, $n=34$, a praga vence o parasito. A inserção de maior número de predadores só adiará sua derrota, veja figura (4.8), onde $G_{0}=100$ e a praga vence em menos de dois meses, $n=50$.

No caso de uma quantidade inicial excessiva de predadores, o sistema altera seu comportamento. Ao invés da trajetória da solução tender para as redondezas do ponto $\left(P_{4}, M_{4}, L_{4}, G_{4}\right)$, ela não se direciona para nenhum equilíbrio, temos $M \rightarrow+\infty$ e $G \rightarrow-\infty$. Portanto os predadores são eliminados fulminantemente pela praga. Veja figura (4.9), onde inserimos 300 predadores, $G_{0}=300$, e a praga vence no período de dois dias. 


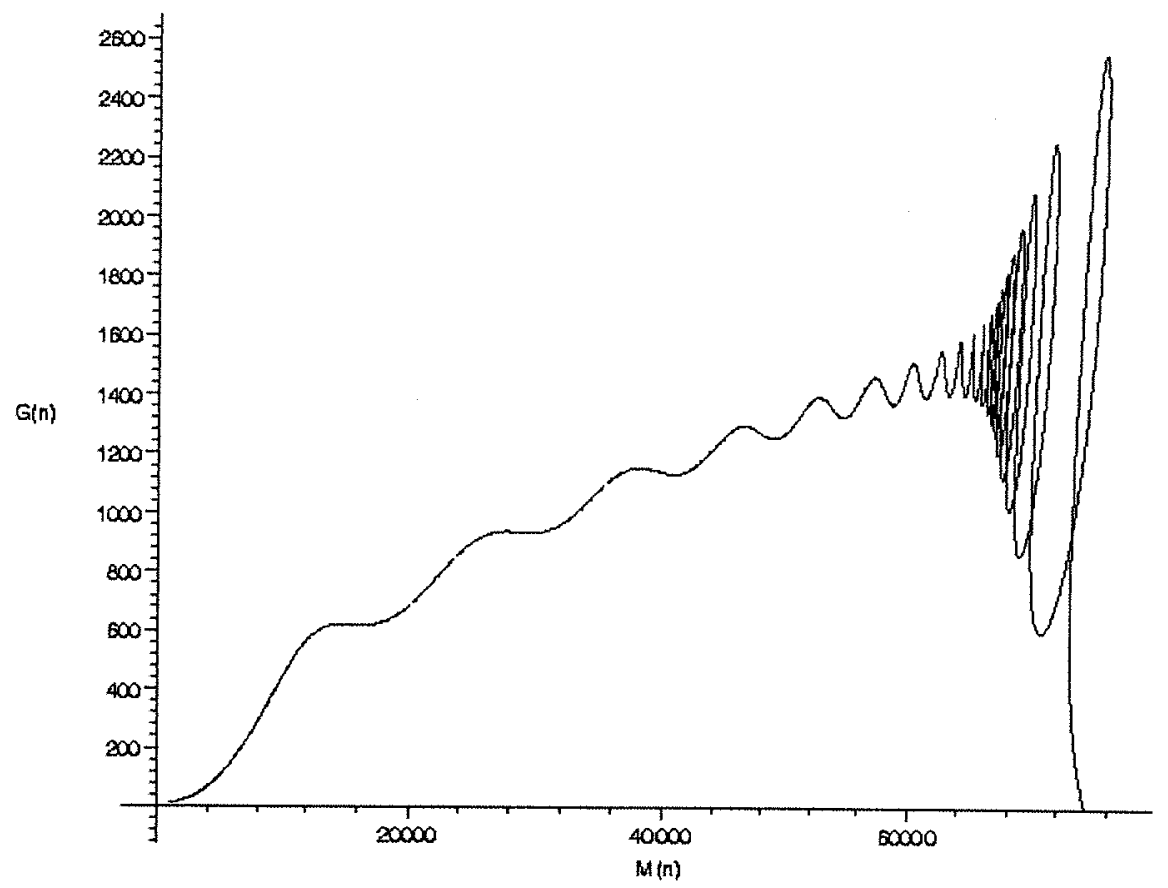

Figura 4.7: $M \times G\left|k<k_{0}\right|$ situação (i) $\left|G_{0}=10\right| n=34$

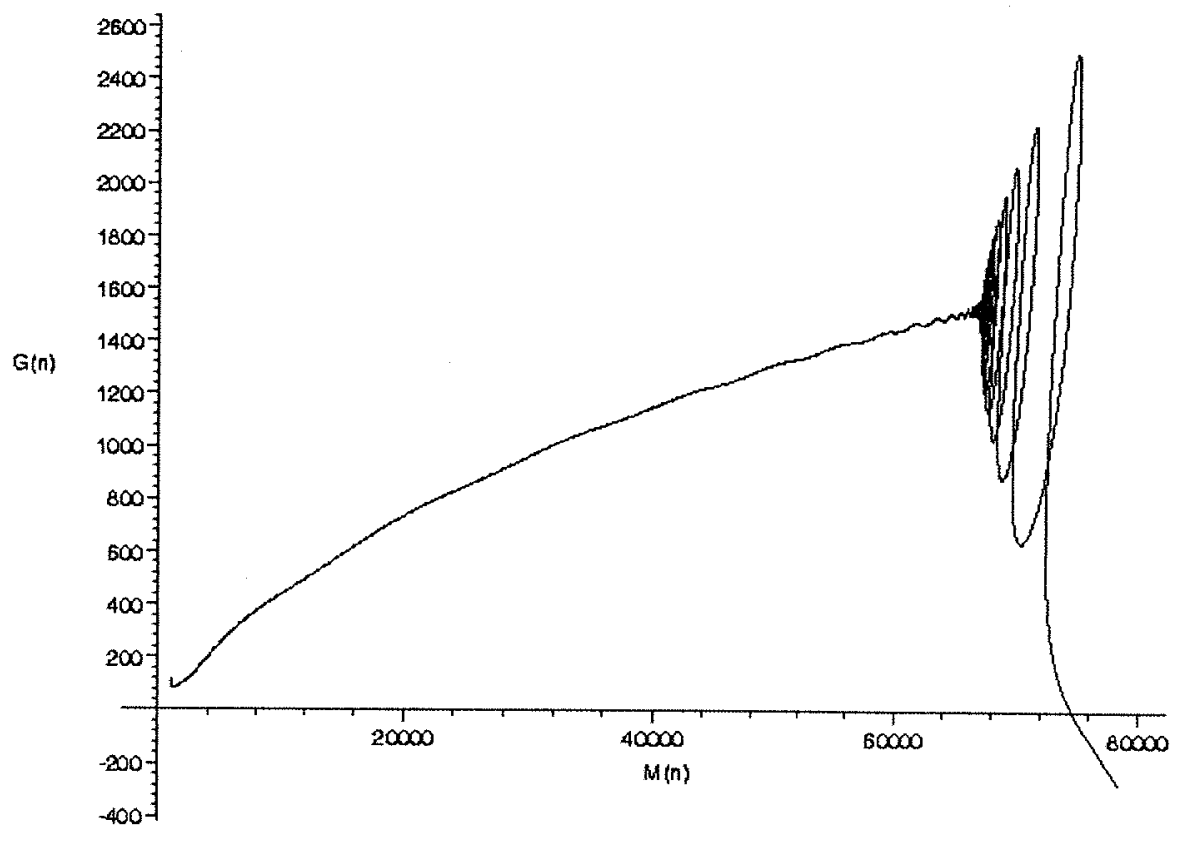

Figura 4.8: $M \times G\left|k<k_{0}\right| \operatorname{situação~(i)~}\left|G_{0}=100\right| n=50$ 


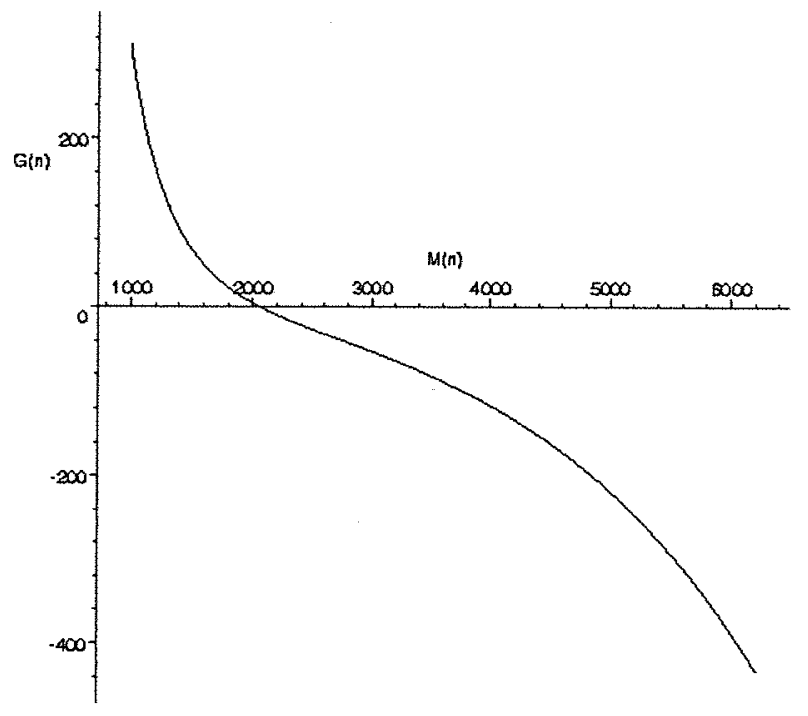

Figura 4.9: $M \times G\left|k<k_{0}\right| \operatorname{situação~(i)~}\left|G_{0}=300\right| n=3$

Nas situações (ii) e (iii) teremos o mesmo tipo de comportamento das populações, com a praga vencendo em tempo cada vez menor e o adicionamento de maior número de predadores sendo ineficaz. As figuras (4.10) e (4.11) representam a dinâmica das populações $M$ e $G$ em cada situação.

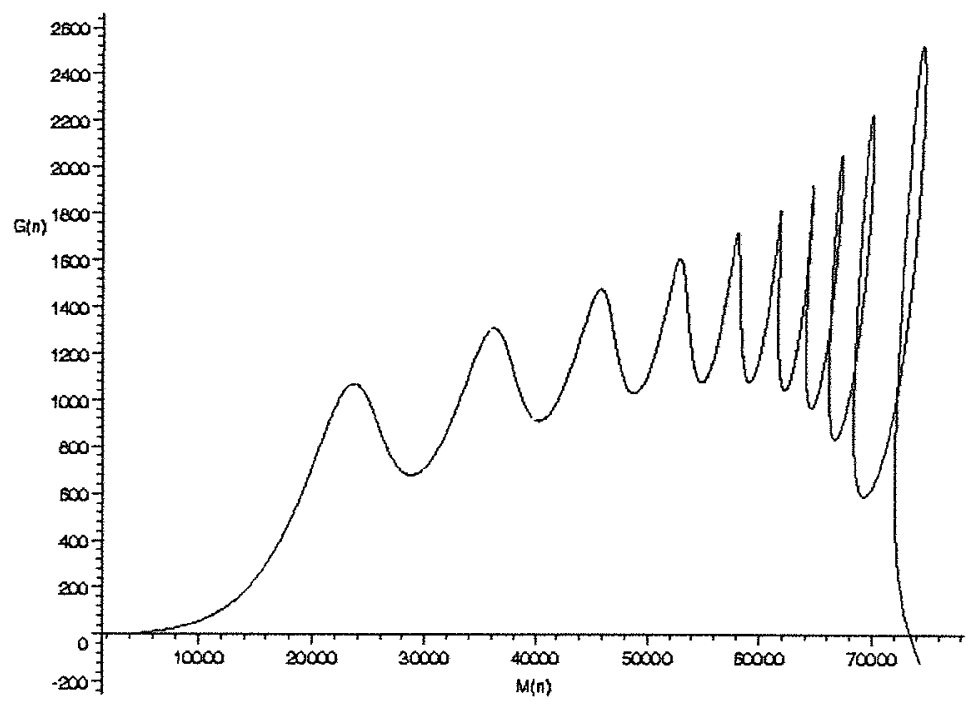

Figura 4.10: $M \times G\left|k<k_{0}\right|$ situação (ii) $\left|G_{0}=10\right| n=18$ 


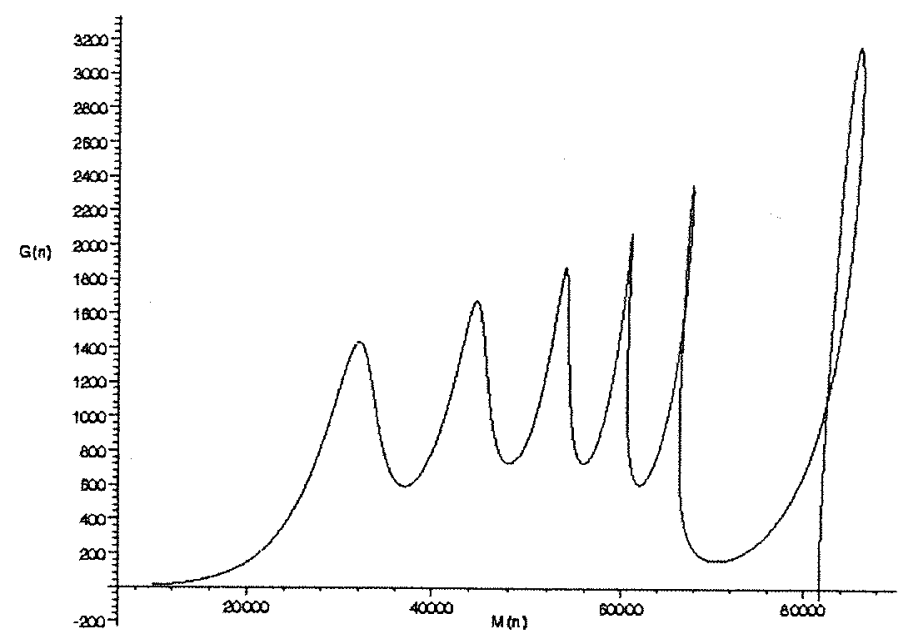

Figura 4.11: $M \times G\left|k<k_{0}\right|$ situação (iii) $\left|G_{0}=10\right| n=11$

$$
\underline{k=.005}
$$

A figura (4.12) representa a dinâmica das populações $M$ e $G$ na situação (i) com a inserção de 10 predadores, $G_{0}=10$, durante 10 meses, $n=300$. Note que a trajetória tende em direção ao equilíbrio $\left(P_{4}, M_{4}, L_{4}, G_{4}\right)$, o que indica que esta trajetória está contida na bacia de atração do ponto $x_{4}$.

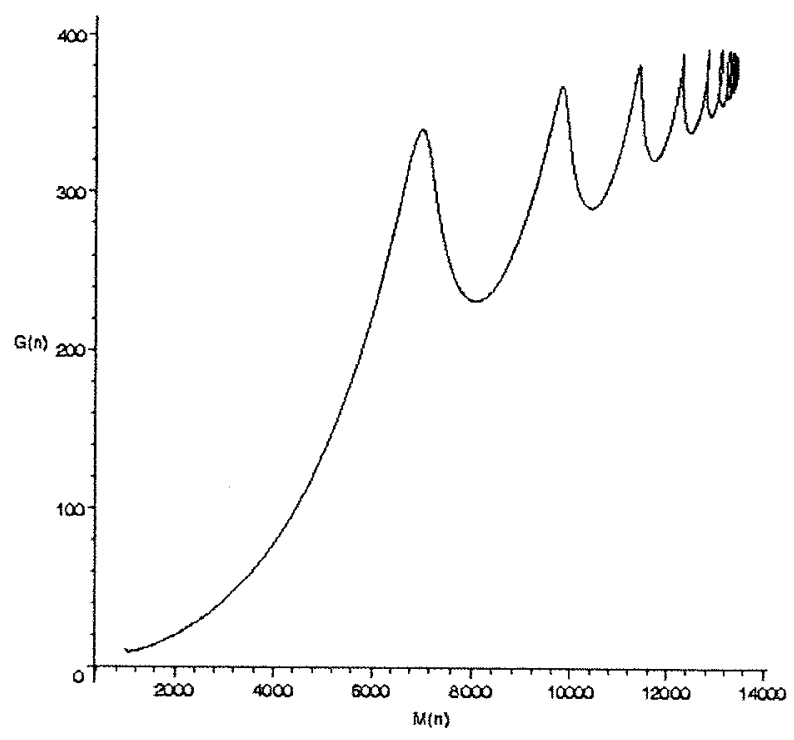

Figura 4.12: $M \times G\left|k>k_{0}\right| \operatorname{situação~(i)~}\left|G_{0}=10\right| n=300$ 
Já a figura (4.13), que representa a dinâmica das populações $M$ e $G$ na situação (ii), $\operatorname{com} G_{0}=10$, mostra que a trajetória da solução é externa a bacia de atração do equilíbrio, causando a morte do predador em aproximadamente três semanas. Neste caso o aumento do número inicial de predadores é eficiente pois levará a trajetória para o interior da bacia de atração e, portanto, para o equilíbrio, veja figura (4.14), onde $G_{0}=30$.

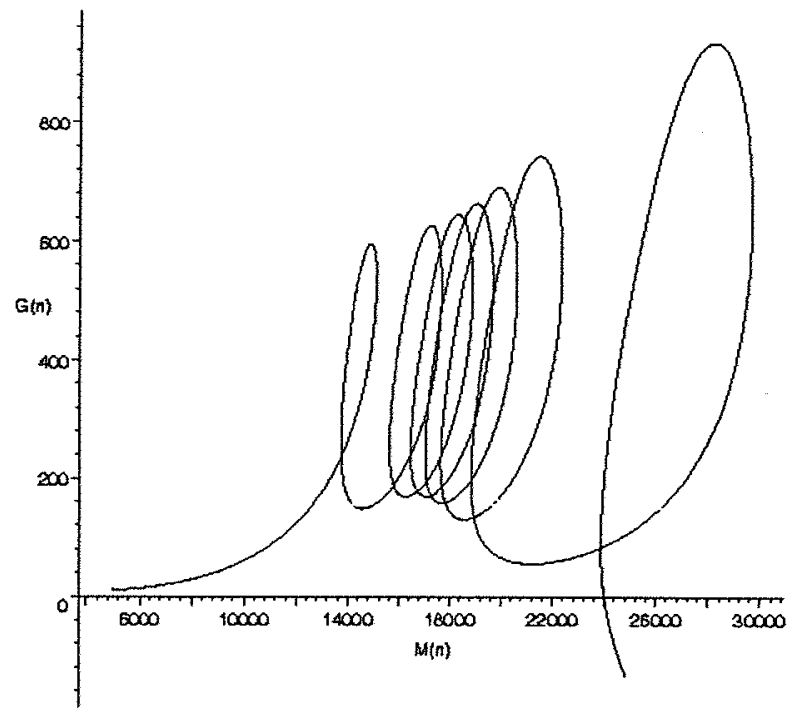

Figura 4.13: $M \times G\left|k>k_{0}\right| \operatorname{situação~(ii)~}\left|G_{0}=10\right| n=21$

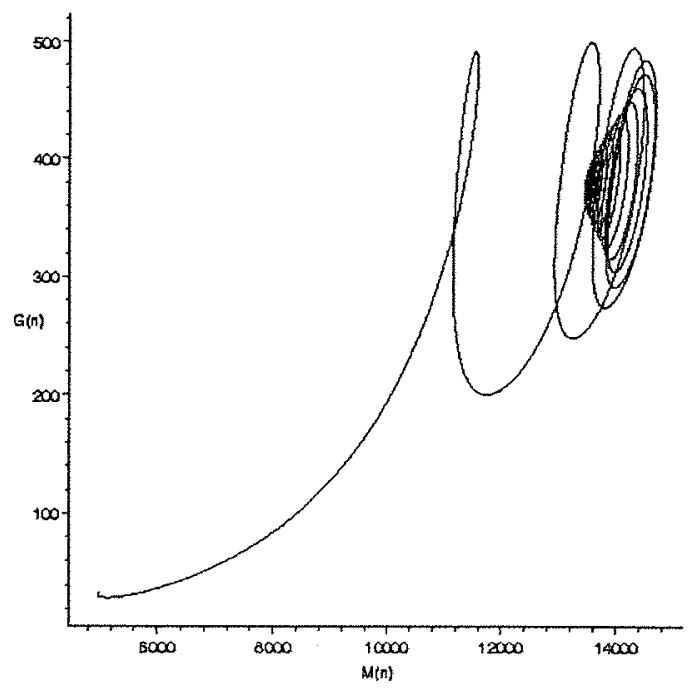

Figura 4.14: $M \times G\left|k>k_{0}\right|$ situação (ii) $\left|G_{0}=30\right| n=300$ 
Do mesmo modo, na situação (iii), a inserção de $G_{0}=30 \mathrm{ou} G_{0}=50$ predadores não é suficiente para controlar a praga, o predador é eliminado no prazo de uma ou duas semanas, respectivamente. Porém, a inserção de $G_{0}=70$ predadores resolve o problema. Veja figuras $(4.15),(4.16)$ e $(4.17)$.

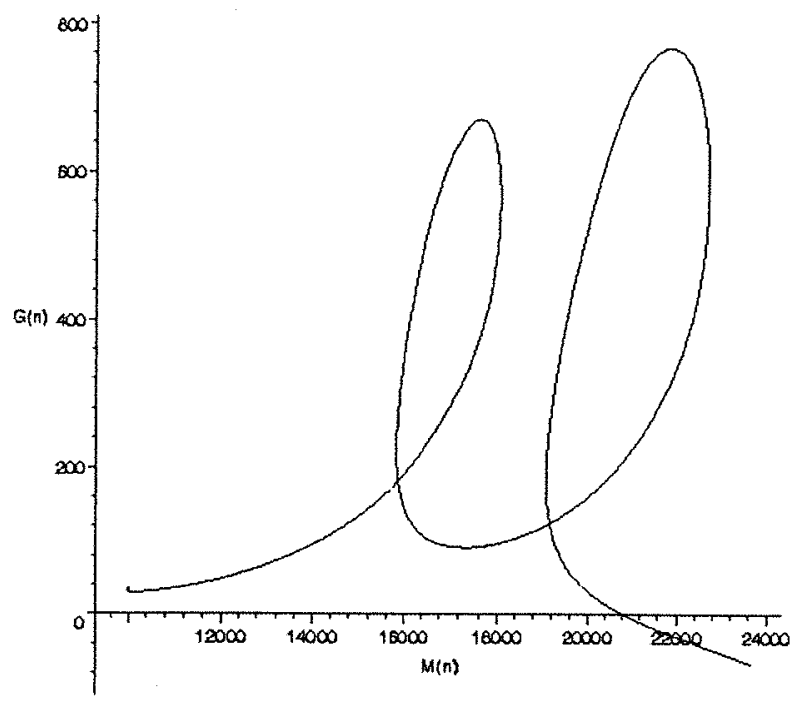

Figura 4.15: $M \times G\left|k>k_{0}\right| \operatorname{situação~(iii)~}\left|G_{0}=30\right| n=7$

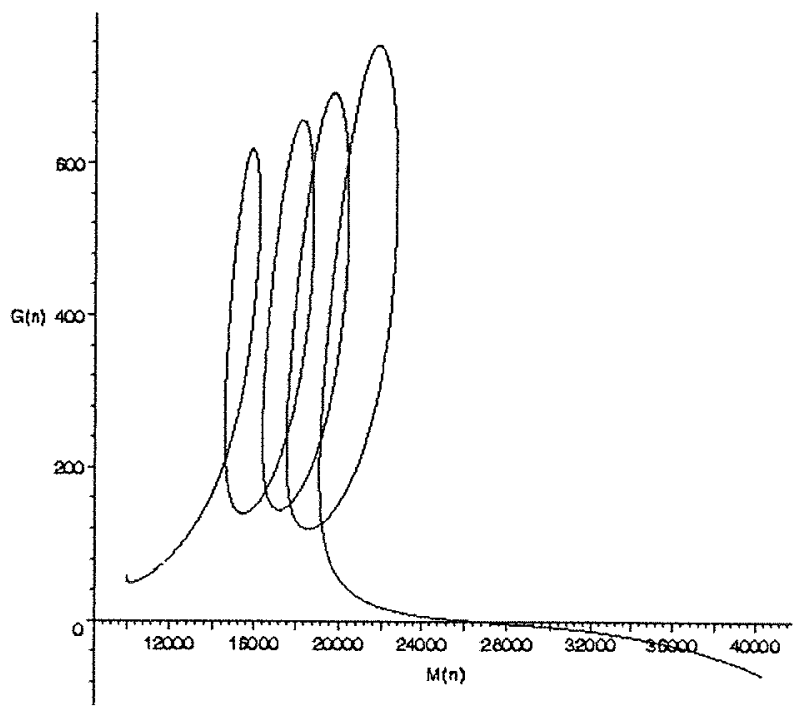

Figura 4.16: $M \times G\left|k>k_{0}\right| \operatorname{situação~(iii)~}\left|G_{0}=50\right| n=14$ 


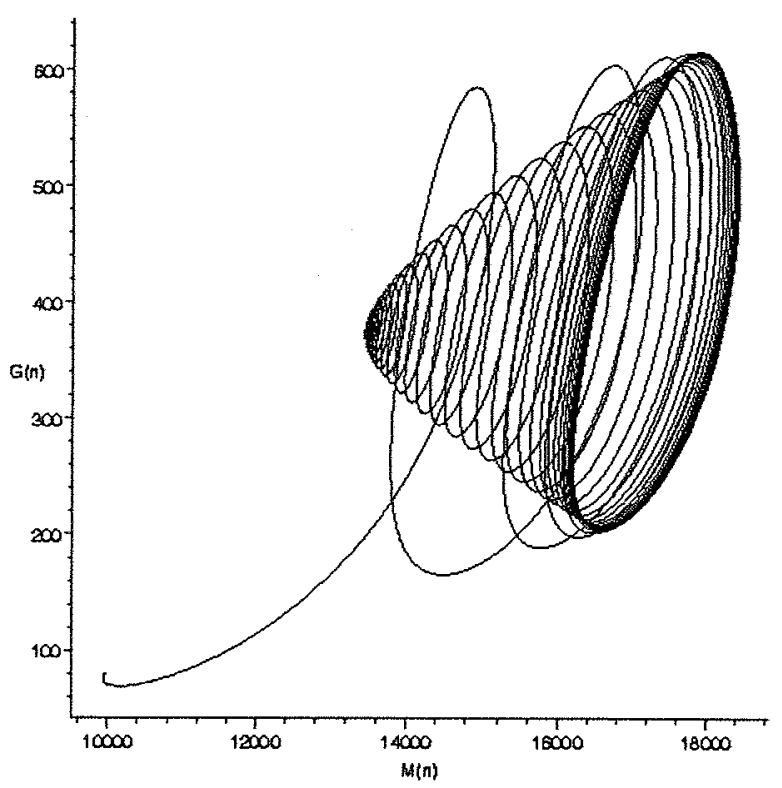

Figura 4.17: $M \times G\left|k>k_{0}\right|$ situação (iii) $\left|G_{0}=70\right| n=300$

Novamente a inserção de um número excessivo de predadores é perigosa. Nesta última situação (iii), por exemplo, variamos o valor de $G_{0}$ e o equilíbrio foi atingido para $G_{0}=500$, apresentando um comportamento análogo a figura (4.17), mas com $G_{0}=600$ tivemos a morte do predador em menos de dois dias, veja figura (4.18).

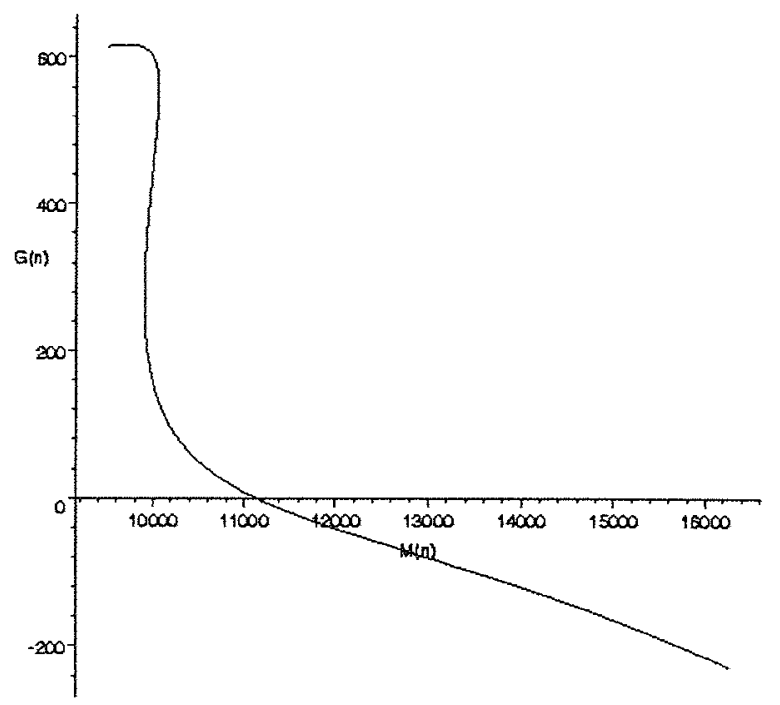

Figura 4.18: $M \times G\left|k>k_{0}\right|$ situação (iii) $\left|G_{0}=600\right| n=2$ 


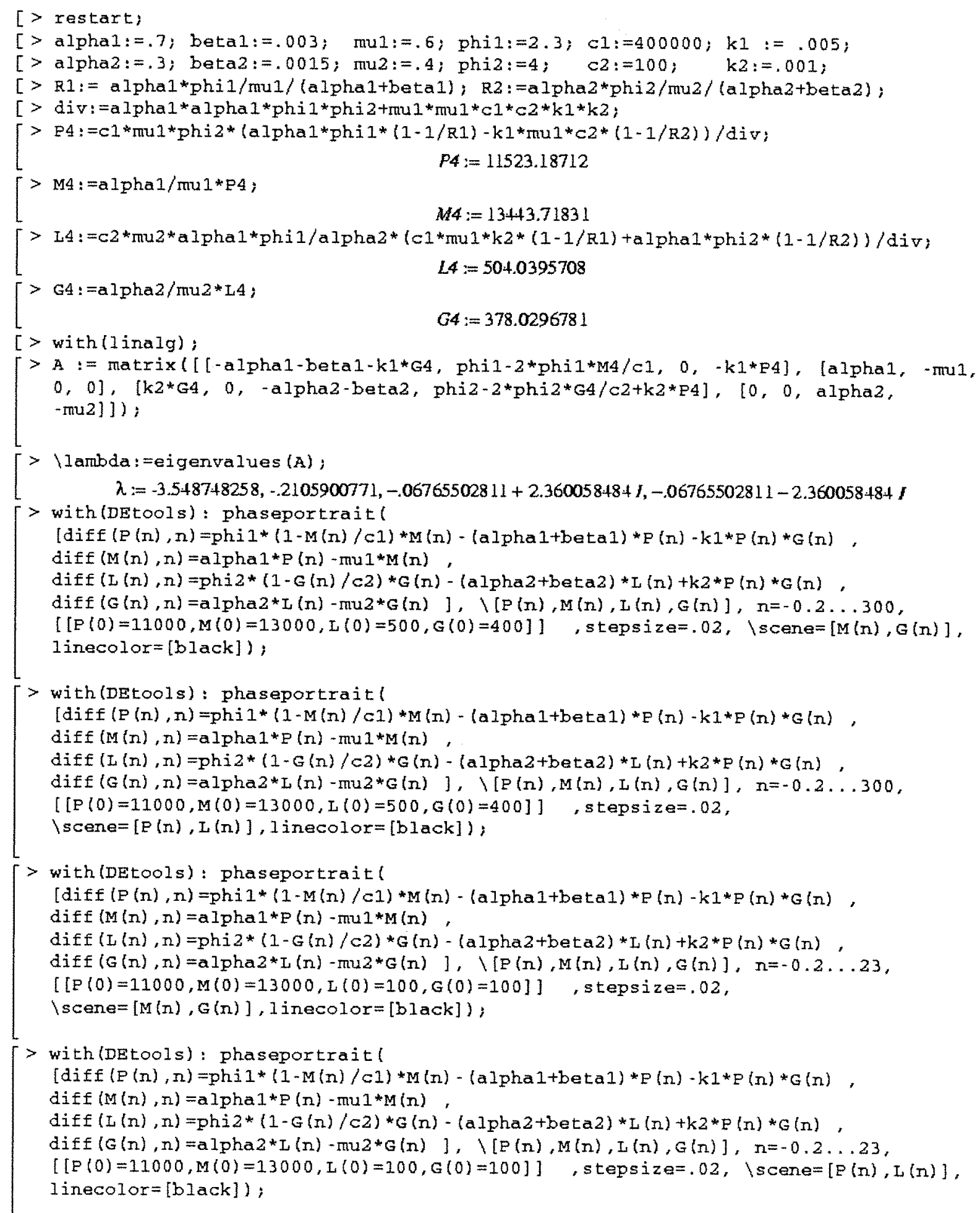

Tabela 4.8: Sequência de comandos do Maple para a plotagem dos gráficos 


\subsubsection{Escolha do vetor $q$}

Ao utilizarmos procedimentos numéricos raramente encontramos soluções exatas, mas sim aproximações destas. Por exemplo, se $\lambda=\omega_{0} i$ é autovalor de $A$ sabemos que também será autovalor da matriz adjunta $A^{t}$. Ao calcularmos numericamente os autovalores de $A$, encontramos $\lambda=2.846703802 i \mathrm{e}$, quando calculamos os autovalores de $A^{t}$, obtemos $\lambda=-.15 \cdot 10^{-8}+2.846703802 i$, veja na tabela (4.4). Claro que para efeitos práticos podemos adotar $-.15 \cdot 10^{-8}$ como sendo igual a zero, desde que todas as outras grandezas dessa ordem passem a ser desprezadas. Outro exemplo na tabela (4.4), quando calculamos $\langle p, q\rangle$ obtemos $1-.243 \cdot 10^{-9} i$, valor este que consideraremos como sendo 1 .

Se escolhermos para o vetor $q$ um tamanho 100 vezes maior que o dado em (4.10), portanto

$$
q=\left(\begin{array}{c}
82055.42609+108077.4610 i \\
29517.56045-13955.88184 i \\
86280.21803+13049.40530 i \\
2601.486634-8727.100717 i
\end{array}\right),
$$

obtemos um valor mais evidente (bem mais positivo) para o coeficiente de Lyapunov,

$$
l_{1}=35.35224196
$$

porém o vetor $p$ aparece com coordenadas da ordem de $10^{-6}$ e algumas funções com valores da ordem de $10^{8}$ e $10^{9}$, veja tabela (4.10).

Por outro lado, se escolhermos para o vetor $q$ um tamanho 100 vezes menor que o dado em $(4.10)$, portanto

$$
q=\left(\begin{array}{c}
8.205542609+10.80774610 i \\
2.951756045-1.395588184 i \\
8.628021803+1.304940530 i \\
.2601486634-.8727100717 i
\end{array}\right)
$$

obtemos um valor para o coeficiente de Lyapunov,

$$
l_{1}=.3535224196 \cdot 10^{-6}
$$

valor esse muito próximo de zero (próximo a $10^{-8}$ ), veja tabela (4.11). 
Há indícios que a dependência de $l_{1}$ em relação ao autovetor $q$ seja quadrática, conforme sugeriram essas últimas simulações. A tabela (4.9), onde $l_{1}$ foi calculado para diferentes tamanhos de $q$, corrobora com esses indícios. Ao gerarmos a tabela (4.9), consideramos para $q=q_{0}$ o valor dado em (4.10) e, para cada $n=-6, \ldots, 7$, fizemos $q_{n}=10^{n} q_{0}$ e calculamos o respectivo $l_{1}^{(n)}$.

\begin{tabular}{c|cr|c}
$n$ & $l_{1}$ & $n$ & $l_{1}$ \\
\hline-6 & $.3535224196 \cdot 10^{-14}$ & 1 & .3535224196 \\
-5 & $.3535224196 \cdot 10^{-12}$ & 2 & $.3535224196 \cdot 10^{2}$ \\
-4 & $.3535224196 \cdot 10^{-10}$ & 3 & $.3535224196 \cdot 10^{4}$ \\
-3 & $.3535224196 \cdot 10^{-8}$ & 4 & $.3535224196 \cdot 10^{6}$ \\
-2 & $.3535224196 \cdot 10^{-6}$ & 5 & $.3535224196 \cdot 10^{8}$ \\
-1 & $.3535224196 \cdot 10^{-4}$ & 6 & $.3535224196 \cdot 10^{10}$ \\
0 & $.3535224196 \cdot 10^{-2}$ & 7 & $.3535224196 \cdot 10^{12}$
\end{tabular}

Tabela 4.9: O coeficiente de Lyapunov para vários tamanhos de $q$

A nossa escolha para o tamanho de $q$ foi no intuito de minimizar as possibilidades de erros numéricos, evitando assim comprometer os resultados obtidos. Um estudo da propagação de erros seria interessante nesse momento, porém a escolha feita para o vetor $q$ nas seções anteriores obteve sempre resultados compreendidos entre $10^{-5} \mathrm{e} 10^{4}$, valores estes que estão distantes da ordem de aproximações feitas. 


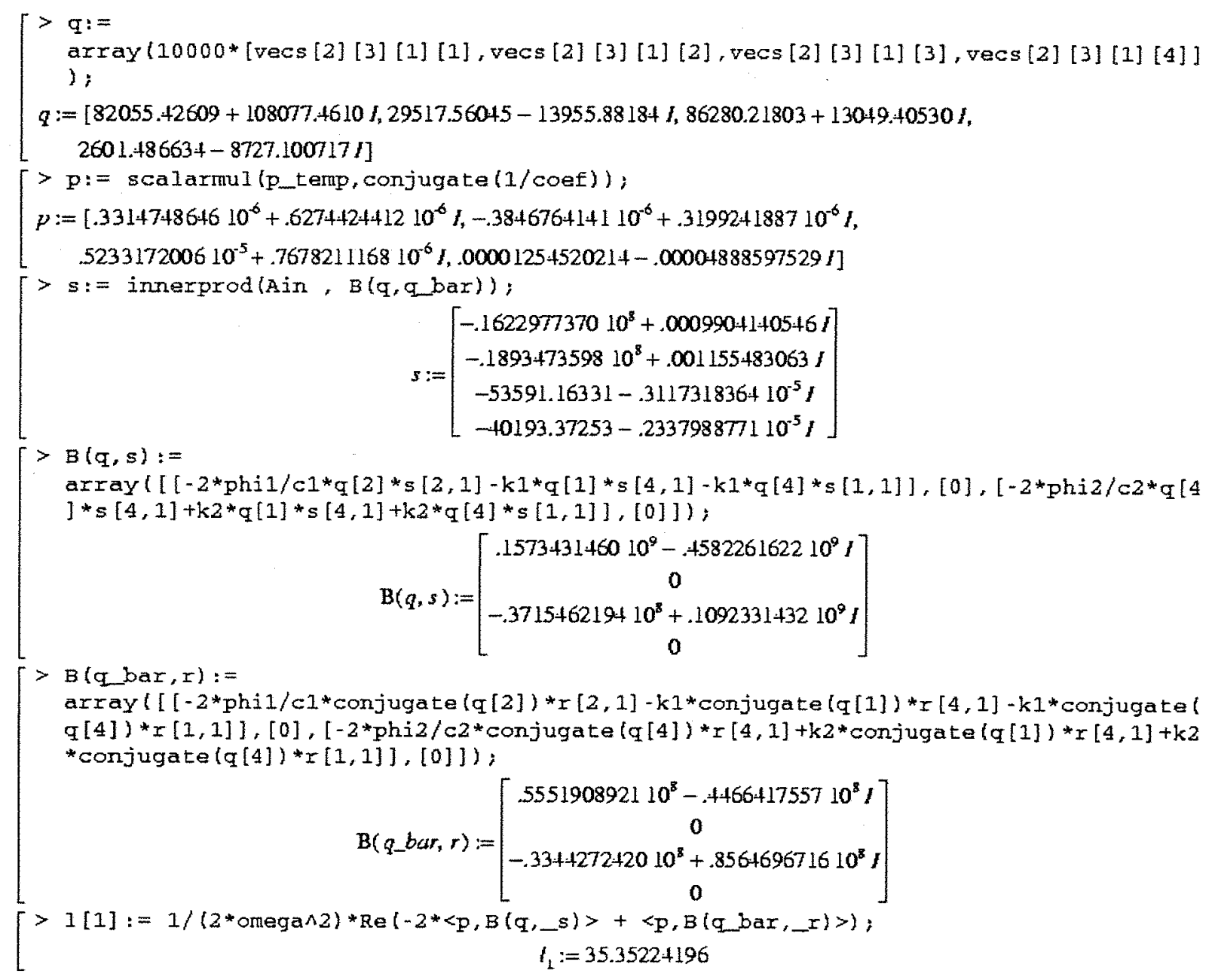

Tabela 4.10: Sequência de comandos com tamanho do vetor $q$ aumentado 


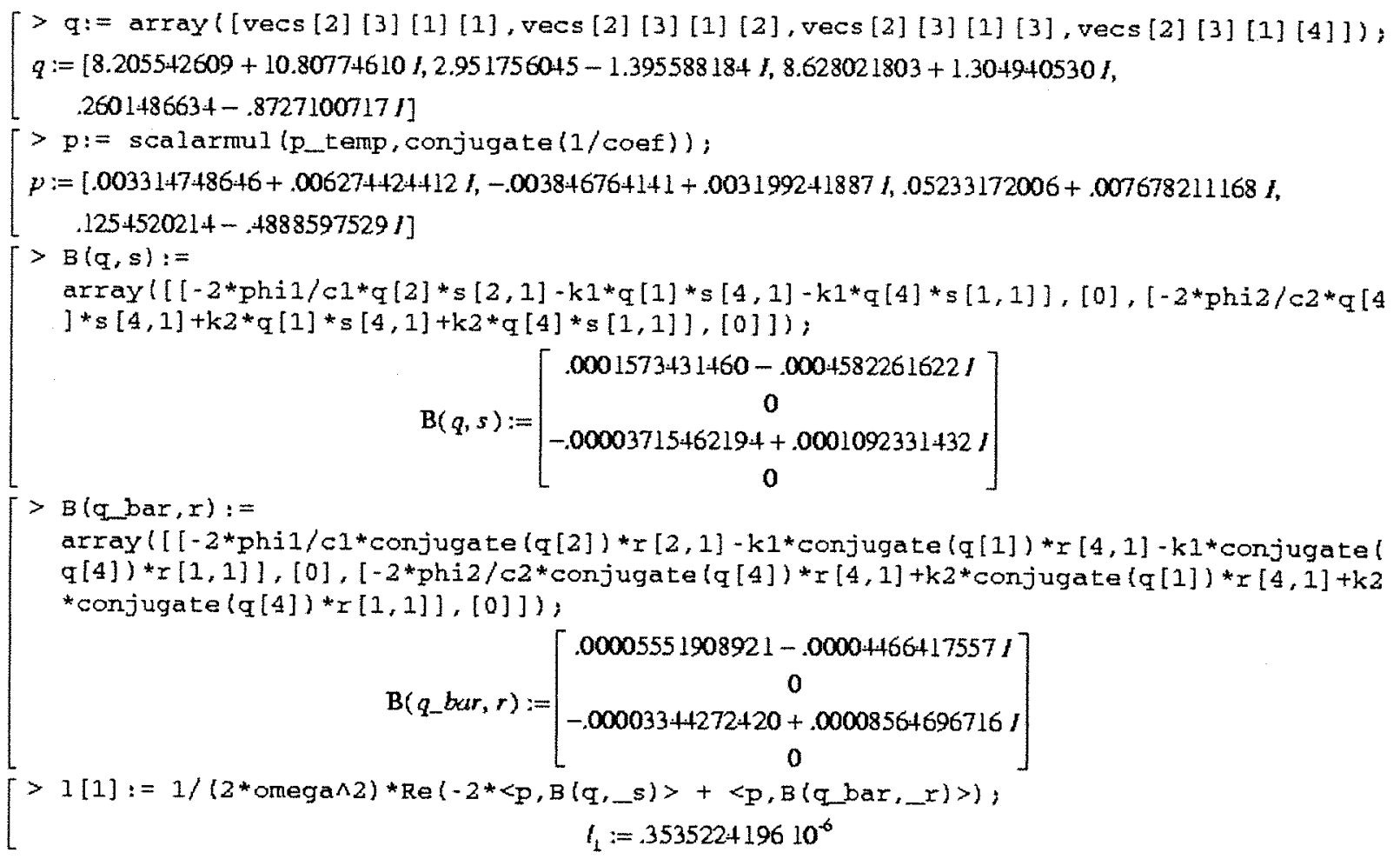

Tabela 4.11: Sequência de comandos com tamanho do vetor $q$ diminuido 


\subsection{Resumo}

As simulações numéricas que fizemos neste capítulo foram de caráter exploratório. Não é nossa intenção, no presente estudo, infiltrarmo-nos pelo campo da Análise Numérica, de modo que utilizamos um pacote computacional fechado (Maple) e simplesmente implementamos as rotinas necessárias. Nosso objetivo foi comprovar a existência de uma bifurcação de Hopf para um dado conjunto de valores atribuídos aos parâmetros. Garantida a existência da bifurcação para este conjunto de valores, espera-se que para outros valores próximos a esses ainda tenhamos o mesmo comportamento. Como este trabalho visou um estudo qualitativo optamos por não estendermos as simulações numéricas para outros valores dos parâmetros, apesar de que nenhum trabalho extra seria necessário.

Com os resultados obtidos na seção anterior, comprovamos que existem valores para os parâmetros $\alpha, \beta, \mu, \phi, c, k, \alpha^{\prime}, \beta^{\prime}, \mu^{\prime}, \phi^{\prime}, c^{\prime}$ e $k^{\prime}$, capazes de satisfazer os casos (a) (ou ( $a_{1}$ ), quando (a) é subdividida em $\left(a_{1}\right),\left(a_{2}\right)$, etc...), da seção 3.3 , e as condições de Hopf, fazendo com que o sistema (S) se comporte conforme a figura (3.2), pág. 80. 


\section{Conclusão}

Neste trabalho, estudamos o sistema (S),

$$
\left\{\begin{array}{l}
\frac{d P}{d t}=\phi\left(1-\frac{M}{c}\right) M-(\alpha+\beta) P-k P G \\
\frac{d M}{d t}=\alpha P-\mu M \\
\frac{d L}{d t}=\phi^{\prime}\left(1-\frac{G}{c^{\prime}}\right) G-\left(\alpha^{\prime}+\beta^{\prime}\right) L+k^{\prime} P G \\
\frac{d G}{d t}=\alpha^{\prime} L-\mu^{\prime} G
\end{array}\right.
$$

que representa o modelo de interação biológica da praga minadora da folha de citros com o seu parasito natural. Detalhes dessa interação são dados no capítulo 1. Esse sistema apresenta quatro pontos de equilíbrio os quais analisamos no capítulo 3. Para três deles, $\left(P_{1}, M_{1}, L_{1}, G_{1}\right),\left(P_{2}, M_{2}, L_{2}, G_{2}\right),\left(P_{3}, M_{3}, L_{3}, G_{3}\right)$, escrevemos as expressões dos autovalores da matriz Jacobiana do sistema, em cada um desses pontos, e concluímos o tipo de comportamento das soluções ao redor desses equilíbrios, em termos dos parâmetros envolvidos. Quanto ao quarto ponto de equilíbrio, fornecemos condições para que este seja um ponto de Hopf e então, no capítulo 4, analisamos estas condições numericamente, concluindo que as soluções desse sistema se comportam, para determinados valores dos parâmetros, como previsto na figura (3.2), pág. 80 .

Para a implementação das simulações numéricas no estudo da bifurcação de Hopf do sistema (S), utilizamos o método de projeção descrito no capítulo 2, seção 2.2. Com o intuito de tornar o capítulo autosuficiente, iniciamos este com o estudo da bifurcação de Hopf em sistemas bidimensionais, definindo a forma normal dessa bifurcação e esboçando as condições para que outros sistemas também a possuam. Seguimos, então, apresentando o método da projeção para sistemas com mais de duas dimensões, onde mostramos como calcular as condições de não degenerescência e de transversalidade, que garantem o surgimento da bifurcação de Hopf para o valor crítico ligado aos autovalores imaginários puros. 
Em resumo, o que mostramos foi a existência, em certas condições, de um ciclo periódico na dinâmica das populações das duas espécies envolvidas no modelo. Biologicamente pode-se então explicar a coexistência das populações de ambas as espécies em situações específicas bem como entender as razões da ineficácia do controle das pragas em outras situações.

Este trabalho contrubui com técnicas da teoria qualitativa das equações diferenciais para a compreensão do estudo biológico aqui tratado. A abrangência que este modelo pode atingir, no caso de outras espécies que possam ser partimentadas em idade infantil e adulta e tenham interação dada pelo contato entre a fase adulta de uma e a fase infantil da outra, aumenta significativamente as contribuições deste estudo. 


\section{Referências Bibliográficas}

[1] Amaro, A. A. e Maia, M. L. e Ghilardi, A. A. [1998], Custos de produção na atual conjuntura da citricultura, In: Seminário Internacional de Citros: tratos culturais, 5., 1998, Bebedouro, SP. Anais, Campinas: Fundação Cargill. p. 481-500.

[2] Boyce, William E. e DiPrima, Richard C. [1988], Equações Diferenciais Elementares e Problemas de Valores de Contorno, Guanabara.

[3] Brasil, Ministério da Agricultura e do Abastecimento. portaria n.65 de 16 de maio de 1996. Oficializa a identificação e necessidade de estudos a respeito da praga "minador da folha de citros", até então desconhecida no país, salientando os danos comumente causados por ela e os locais onde foi encontrada oficialmente. Diário Oficial, República Federativa do Brasil, Brasília, DF, v. 124, n. 96, p. 08679, 20 de maio de 1996, seção 1.

[4] Chagas, M. C. M. [1999], Phyllocnistis citrella Stainton, 1856 (Lepidoptera: Gracillariidae): bioecologia e relação com o cancro cítrico, tese de Doutorado em Ciências, Escola Superior de Agronomia Luís de Queiroz, Universidade de São Paulo, Piracicaba.

[5] Cobo Nuñez, G. M. [1996], Ciclo biologico del minador de las hojas de los citricos Phyllocnistis citrella Staiton (Lepidoptera: Gracillariidae) y su relación con sus hospedeiros y enemigos naturales en el Valle del Cauca, tese, Universidade Nacional da Colombia, Palmeira, Colombia.

[6] Coelho, Flávio Ulhoa e Lourenço, Mary Lilian [2001], Um curso de Álgebra Linear, Edusp.

[7] Fundecitrus [1997], Manual técnico do cancro cítrico, Araraquara. 
[8] Fundecitrus. Estatísticas do cancro cítrico, disponível em $<$ http:www.fundecitrus.com.br/escancro.html $>$.

[9] Gravena, S. [1994], Minadora das folhas de citros: a mais nova ameaça da citricultura brasileira, Laranja, Cordeirópolis, v. 15, n. 2, p. 397-404.

[10] Gravena, S. [2000], Manejo integrado de pragas dos citros no contexto da citricultura integrada, In: Seminário Internacional de Citros: produção integrada, 6. Bebedouro, SP. Anais, Campinas: Fundação Chargill, p. 145-175.

[11] Hyun Yang, M. e Ternes, S. [1999], Estudo dos efeitos de dinâmica vital num modelo de controle biológico de pragas, revista de Biomatemática, Campinas, Unicamp/IMECC, v. 9 , p. 58-72.

[12] Kuznetsov, Yuri A. [1998], Elements of Applied Bifurcation Theory, Springer.

[13] LaSalle, J. e Peña, J. E. [1997], A new species of Galeopsomyia: a fortuitous parasitoid of the citrus leafminer, Phyllocnistis citrella., Florida Entomologist, v. 80, p. 461-470.

[14] Lima, Elon Lages [2000], Curso de Análise vol.2, Projeto Euclides.

[15] Pontryagin, L. S. [1962], Ordinary Differential Equations, Addison-Wesley Publishing Company.

[16] Resolução $N^{\circ} 1$ da Comissão Executiva Estadual da CANECC, publicada em 20/03/2000, que trata das incidências da doença cancro cítrico, em propriedades comerciais.

[17] Sotomayor, Jorge [1979], Lições de Equações Diferenciais Ordinárias, Projeto Euclides.

[18] Sotomayor, Jorge [1981], Curvas Definidas por Equações Diferenciais no Plano.

[19] Ternes, Sônia [2001], Modelagem e simulação da dinâmica populacional da larvaminadora-da-folha-de-citros em interação com seus inimigos naturais, Tese de Doutorado, Faculdade de Engenharia Elétrica e de Computação, Unicamp, Campinas, São Paulo. 